Aus dem Institut für Ethik und Geschichte der Medizin

(Prof. Dr. med. C. Wiesemann)

der Medizinischen Fakultät der Universität Göttingen

\title{
WORT ODER UNWORT? \\ Eine Analyse der Metapher Medizintourismus in der ethischen Debatte
}

\author{
INAUGURAL - DISSERTATION \\ zur Erlangung des Doktorgrades \\ der Medizinischen Fakultät der \\ Georg-August-Universität zu Göttingen
}

vorgelegt von

Merle Annika Michaelsen

aus

Lüneburg

Göttingen 2015 
Dekan:

Prof. Dr. rer. nat. H. K. Kroemer

I. Berichterstatterin: Prof. Dr. med. C. Wiesemann

II. Berichterstatterin: Prof. Dr. med. A. Buyx

III. Berichterstatter: Prof. Dr. sc. hum. T. Friede

Tag der mündlichen Prüfung: $\quad$ 24.05.2017 


\section{INHALTSVERZEICHNIS}

1 Einleitung: Medizintourismus - der Ausdruck im Kontext................................................................

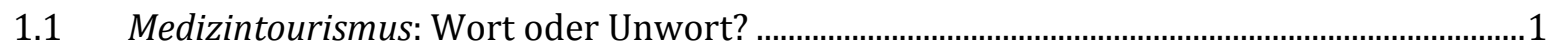

1.2 Metaphern und ihre Bedeutung für die ärztliche Tätigkeit ...........................................................

1.3 Die Morphologie der Metapher Medizintourismus ………………….......................................

1.4 Der Anwendungsrahmen des Terminus …….............................................................................

1.5 Medizintourismus: Explikationen aus der fachlichen Diskussion .............................................8

$1.6 \quad$ Kritische Stimmen aus dem Diskurs...................................................................................... 10

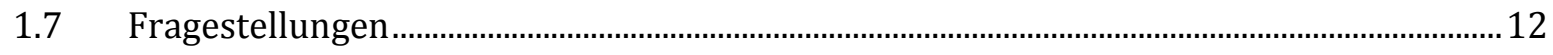

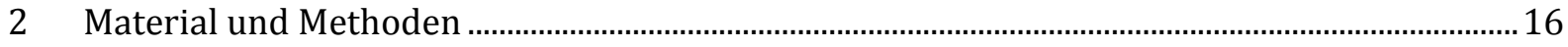

3 Grundlagen des Metaphernbegriffs ............................................................................................ 18

$3.1 \quad$ Von Ivor Richards zu Max Black.............................................................................................

3.2 László Kovács: die verschiedenen Leistungen einer Metapher.............................................. 21

3.2.1 Die illustrative Leistung ................................................................................................... 21

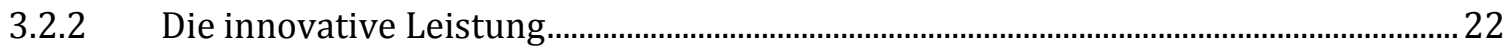

3.2.3 Die konstitutive Leistung........................................................................................... 22

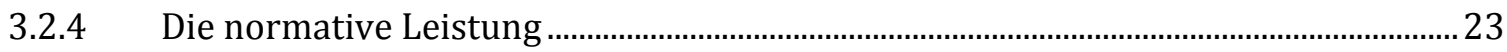

3.2.5 Verdeutlichung der Leistungen einer Metapher anhand des Beispiels „Der Mensch

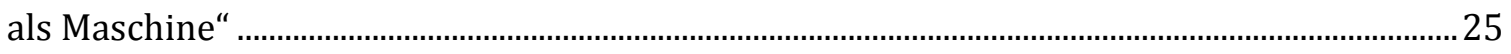

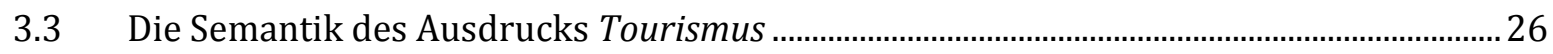

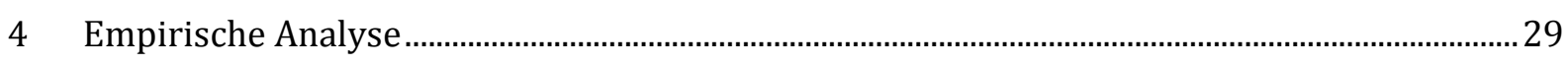

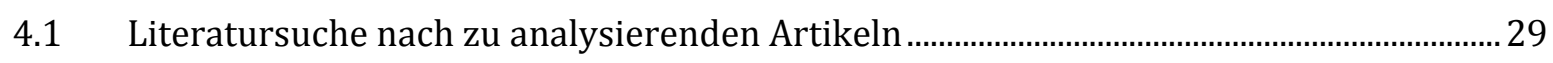

4.2 Quantitative und qualitative Analyse mit atlas.ti .................................................................... 30

4.3 Medizintourismus - Reproduktionstourismus - Transplantationstourismus Eine

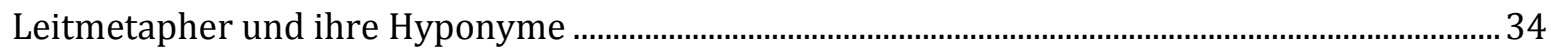

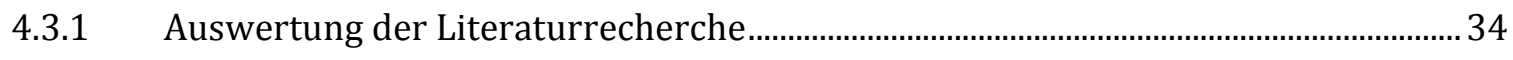

4.3.2 Auswertung des Textkorpus ……………....................................................................... 36

4.4 Medizintourismus: ein Ausdruck - vielfältige Bedeutungen .................................................... 40

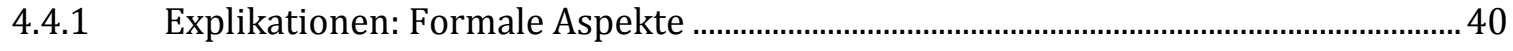

4.4.2 Explikationen: Inhaltliche Aspekte................................................................................. 41

4.4.3 Explikationen: Inhaltliche Aspekte in Abhängigkeit von den Hyponymen ................ 44

4.5 Semantische Aspekte von Tourismus im Kontext von Medizintourismus............................. 46

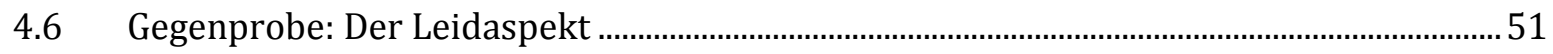


5 Bedeutungen und Nebenbedeutungen der Metapher Medizintourismus . 55

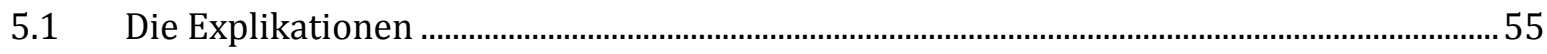

5.1.1 Mehrdeutigkeit - illustriert am Beispiel von Transplant Tourism ................................ 56

5.1.2 Eine Leitmetapher - viele Ausdrücke..................................................................................62

5.2 Ortswechsel - Freizeit - Wirtschaft: Semantische Felder von Tourismus im

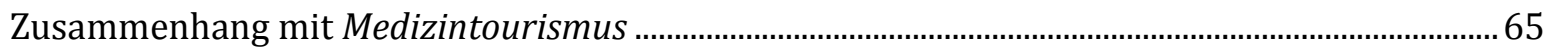

5.3 Die Leistungen der Metapher Medizintourismus ………......................................................... 67

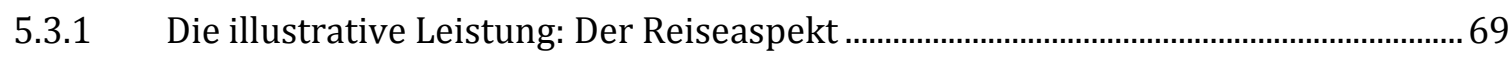

5.3.2 Die innovative Leistung: Der Wirtschaftsaspekt ............................................................ 70

5.3.3 Die konstitutive Leistung: Der Freizeitaspekt/Die Perspektive der Patientinnen. 72

5.3.4 Der Leidaspekt: Von der innovativen zur konstitutiven Leistung................................ 75

6 Ethische Analyse der normativen Verwendung der Metapher Medizintourismus..................... 79

$6.1 \quad$ Normative Verwendungsweisen............................................................................................ 79

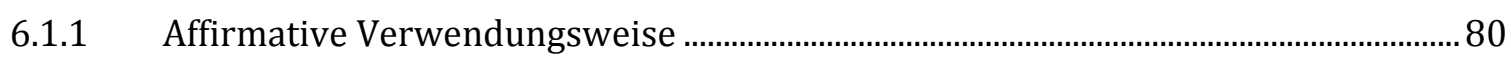

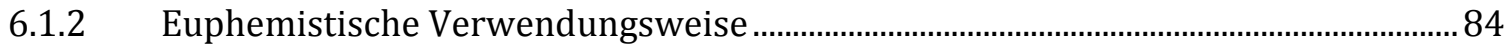

6.1.3 Pejorative Verwendungsweise................................................................................... 85

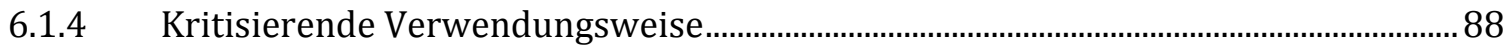

6.2 Auswirkungen der Metapher Medizintourismus auf unser Verständnis von einer

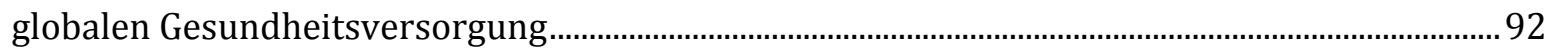

6.2.1 Gesundheitsversorgung: Wirtschaftszweig oder soziales System? .............................. 92

6.2.2 Touristin - Kundin - Patientin? ………………....................................................................93

$7 \quad$ Auf der Suche nach einem alternativen Ausdruck ….........................................................................99

7.1 Anforderungen an einen alternativen Ausdruck .......................................................................99

7.2 Vorschläge aus dem Diskurs .....................................................................................................102

8 Schlussfolgerung und Ausblick...................................................................................................106

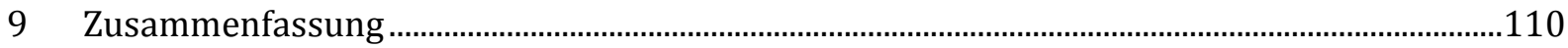

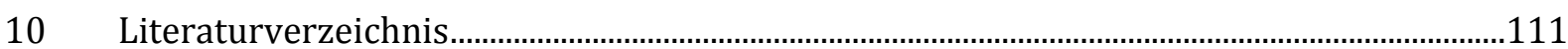

Lebenslauf. ..Fehler! Textmarke nicht definiert.

Danksagung 


\section{EINLEITUNG: MEDIZINTOURISMUS - DER AUSDRUCK IM KON- TEXT}

\subsection{MEDIZINTOURISMUS: WORT ODER UNWORT?}

Im Jahr 2007 wurde der Ausdruck Sterbetourismus zum Schweizer Wort des Jahres gewählt. Laut einem Artikel der Online-Ausgabe der Neuen Zürcher Zeitung räumte die Jury in ihrer Pressemitteilung ein, der Ausdruck möge zunächst pietätlos erscheinen. Sie begründete ihre Wahl jedoch damit, dass eine breite öffentliche Diskussion über „aktive und passive Sterbehilfe in der Schweiz erst mit den gehäuften Besuchen von Menschen aus dem Ausland“ eingesetzt habe (NZZ online 2007).

In der Schweiz wird wie in Deutschland nicht nur ein Wort des Jahres, sondern auch ein Unwort des Jahres gewählt. Das Wort des Jahres sollte wichtig, häufig, von besonderer Bedeutung und von sprachlicher Qualität sein. Zum Unwort des Jahres hingegen werden „sprachliche Missgriffe“ bestimmt, die „sachlich grob unangemessen sind und möglicherweise sogar die Menschenwürde verletzen“ (Website der Aktion „Schweizer Wort des Jahres“). Tatsächlich wurde in Deutschland 2013 das Wort Sozialtourismus zum Unwort des Jahres gewählt, unter anderem mit folgender Begründung: „Das Grundwort Tourismus suggeriert in Verdrehung der offenkundigen Tatsachen eine dem Vergnügen und der Erholung dienende Reisetätigkeit“ (Janich 2014). Diese Kritik trifft durchaus auf die Situation von vielen Menschen zu, die zu medizinischen Zwecken reisen. Versetzt man sich in die Lage einer Person, die in die Schweiz reist, um Hilfe beim Sterben zu erhalten, weil dies im Heimatland nicht möglich ist, liegt der Gedanke nahe, Sterbetourismus könnte sachlich grob falsch sein. Hätte Sterbetourismus also nicht auch zum Unwort des Jahres gekürt werden können? Bereits 2002 hatte schließlich der Bioethiker Guido Pennings die Verwendung des Ausdrucks Reproduktionstourismus kritisiert, da er das Phänomen zu einseitig beschreibe und Assoziationen zu Begriffen wie Urlaub, Freizeit und Spaß wecke, die die Realität der Betroffenen nicht widerspiegele (Pennings 2002). Dies trifft auf sogenannte Sterbetouristinnen ${ }^{1}$ mit Sicherheit ebenso zu.

\footnotetext{
${ }^{1}$ Die weiblichen Personalformen Touristin, Ärztin, Patientin etc. stehen in allgemeinen Passagen, die sich nicht auf konkrete Personen beziehen, für geschlechtsunabhängige Bezeichnungen. Zur Ermöglichung einer besseren Lesbarkeit verwende ich - entgegen der Empfehlung für geschlechtergerechte Sprache des Gleichstellungsbüros der Universität Göttingen, das männliche und das weibliche Geschlecht explizit zu benennen (zum Beispiel Ärztinnen und Ärzte) - die weibliche Form. Nur wenn das biologische Geschlecht
} 
Beide Ausdrücke, Sterbetourismus und Reproduktionstourismus, sind auf den übergeordneten Ausdruck Medizintourismus zurückzuführen. Dabei handelt es sich um eine Metapher, also um einen bekannten Ausdruck, der zur Benennung und Illustration eines zuvor unbekannten Sachverhaltes herangezogen wird. Während die einen die Metapher Sterbetourismus als besondere sprachliche Errungenschaft hervorheben, kritisieren die anderen den Begriff Reproduktionstourismus und fordern, diesen Ausdruck zu ersetzen. Gibt es einen sprachlichen Zusammenhang zwischen Medizintourismus, Sterbetourismus und Reproduktionstourismus? Gibt es noch weitere Tourismus-Ausdrücke, die in diesem Kontext eine Rolle spielen? Was verstehen wir überhaupt unter Medizintourismus? Und welche ethischen Argumente können für, welche gegen die Verwendung des Ausdrucks vorgebracht werden?

In dieser Arbeit werden die unterschiedlichen Definitionen und Konnotationen der Metapher Medizintourismus herausgearbeitet, und es soll insbesondere kritisch gefragt werden, ob dieser Ausdruck für die ethische Fachdiskussion geeignet ist. Zunächst wird jedoch zum besseren Verständnis ein Überblick über das als Medizintourismus bezeichnete Phänomen gegeben.

Wie facettenreich dieses Phänomen ist, lässt sich an zwei Studien zeigen, die zu sehr unterschiedlichen Schlussfolgerungen kommen. Laut einer amerikanischen Studie von 2011 reisen etwa 13.500 US-Amerikanerinnen jährlich mit Hilfe einer kommerziellen Vermittlung ins Ausland, um dort medizinische Behandlungen durchführen zu lassen. In dieser Gruppe Reisender sind die am häufigsten vorgenommenen Behandlungen orthopädische Operationen, zum Beispiel Alloplastiken von Hüfte oder Knie, gefolgt von Bypass-Operationen und reproduktionsmedizinischen Behandlungen. Nach Auskunft der Vermittlungen seien die niedrigeren Kosten der größte Motivationsfaktor für Betroffene, Behandlungen im Ausland durchführen zu lassen (Alleman et al. 2011).

Die Soziologinnen Nicky Hudson und Lorraine Culley konnten in einer Studie mit Patientinnen und Patienten aus Großbritannien, die für eine reproduktionsmedizinische Behandlung ins Ausland gereist sind, dagegen zeigen, dass diese zumeist ohne kommerzielle Vermittlung Reise und Behandlung organisierten. Außerdem werde nicht ein einzelner Grund, sondern in den meisten Fällen eine Kombination aus push and pull factors als Motivation genannt, eine ART (assisted

der Protagonistinnen von Bedeutung ist, zum Beispiel bei der Vorstellung eines bestimmten Studiendesigns, erfolgt eine Doppelnennung wie Patientinnen und Patienten. Des Weiteren habe ich möglichst oft Bezeichnungen gewählt, die keine Geschlechterzuordnung vornehmen, wie Personal oder Erkrankte. Eine geschlechterneutrale Sprache allein reicht nicht aus, das Gedankenkonzept der Rezipientin zu verändern, da wir gewohnt sind, bei Pluralbezeichnungen an Prototypen zu denken, die männlich konnotiert beziehungsweise traditionellen Geschlechterrollen verbunden sind (zum Beispiel: die Krankenschwester, der Arzt). Um diese Konzepte/Denkgewohnheiten aufzubrechen/zu hinterfragen, verwende ich in diesem Text die weibliche Form. 
reproductive treatment) im Ausland durchführen zu lassen. Darunter fielen unter anderem die Faktoren verkürzte Wartezeiten, niedrigere Kosten und bessere Behandlungsergebnisse im Zielland (Hudson und Culley 2011).

Anhand dieser beiden Studien lässt sich zeigen, wie unzureichend die gegenwärtige Datenlage zur Beschreibung und Einordnung des Phänomens reisender Patientinnen und Patienten ist: Während die US-amerikanische Studie nur diejenigen erfasst, die mit kommerzieller Vermittlung ins Ausland gehen, stellt die zweite Studie fest, dass in Großbritannien die meisten Patientinnen solche Vermittlungsdienste gar nicht in Anspruch nehmen. Einige Studien konzentrieren sich auf die Nationalität der Teilnehmenden, andere beleuchten nur ein Fachgebiet (zum Beispiel die Reproduktionsmedizin). Es lässt sich jedoch immerhin so viel erkennen, dass sich hinter dem Ausdruck Medizintourismus ein komplexes Phänomen verbirgt, das unterschiedliche Fachbereiche umspannt. So liegt es auf der Hand, dass Kosmetiktourismus und Sterbetourismus Ausdrücke für Teilbereiche von Medizintourismus sind, die sehr unterschiedliche ethische Fragestellungen mit sich bringen. Spätestens jetzt stellt sich die Frage, ob die Grundbedeutungen des Begriffs Tourismus, also seine Denotationen², in dem Phänomen der reisenden Patientinnen wirklich eine so große Rolle spielen, dass dies die Verwendung der Metapher als übergeordneten Ausdruck rechtfertigt.

\subsection{METAPHERN UND IHRE BEDEUTUNG FÜR DIE ÄRZTLICHE TÄTIGKEIT}

Im Klinikalltag von Ärztinnen spielt Kommunikation eine herausragende Rolle. Ärztinnen kommunizieren mit Kolleginnen, Pflegepersonal, Vorgesetzten, Angehörigen und Patientinnen. Sprache nimmt in der Kommunikation eine zentrale Position ein. Ärztinnen müssen zwischen Fachund Alltagssprache hin- und herwechseln, außerdem sollten sie Form und Inhalt eines Gesprächs an Fähigkeiten und Person ihrer Patientinnen anpassen können. Dabei kommt es häufig auf die richtige Wortwahl an. Während „Krebs“ bei einer Person Angst auslöst, weil sie das Wort mit Leid, Tod und Sterben verknüpft, kann eine andere Person dankbar für deutliche Worte sein, etwa weil sie sonst in Sorge wäre, nicht vollständig informiert worden zu sein. Dasselbe Wort kann bei verschiedenen Gesprächsteilnehmenden sowie in unterschiedlichen Kontexten voneinander abweichende Assoziationen hervorrufen. Einzelne Wörter können als verletzend, überfordernd, unsachlich oder diskriminierend empfunden werden. Ärztinnen müssen deshalb ihre Worte mit Bedacht wählen und deren Wirkung auf den Gesprächsverlauf reflektieren.

\footnotetext{
${ }^{2}$ Als Denotationen werden die Grundbedeutungen eines Ausdrucks bezeichnet. Hiermit sind die kontextunabhängigen Bedeutungen gemeint. Davon abzugrenzen sind die Konnotationen, Nebenbedeutungen eines Ausdrucks, die häufig subjektiv und kontextabhängig sind. Beispiel: die Denotation von Esel ist Huftier, eine Konnotation von Esel ist dummer Mensch.
} 
Insbesondere neue, in den öffentlichen Medien kontrovers diskutierte Ausdrücke, wie Medizintourismus beziehungsweise Reproduktionstourismus sollten deshalb nicht unreflektiert in den eigenen Wortschatz übernommen werden. So kann sich zum Beispiel ein unfruchtbares Paar, das sich nach einer bestimmten Fertilitätsbehandlung im Ausland erkundigt, durch eine Gegenfrage wie „Meinen Sie Reproduktionstourismus?“ missverstanden fühlen, da es den Ausdruck als nicht zutreffend für seine Situation empfindet. Tourismus könnte in ihren Augen Urlaub bedeuten, sie verbinden den Ausdruck vielleicht mit Sonne, Strand und Meer, nicht aber mit Krankenhausaufenthalt und medizinischer Behandlung. Vielleicht hat dieses Paar jahrelange Leiderfahrung durch medizinische Eingriffe und Rückschläge nach Therapieversuchen hinter sich; es ist vorstellbar, dass sie ihre Situation mit dem Ausdruck Tourismus als nicht angemessen erfasst sehen.

Unterschwellig normative, als wertend empfundene Aussagen sind im Ärztin-Patientin-Gespräch nicht zielführend, da sie die Patientin unbemerkt kränken und der Vertrauensbeziehung schaden können. Die Wortwahl durch die Ärztin - und insbesondere die Wahl möglicher Metaphern - muss gut überlegt sein, um die Patientin nicht durch implizite Werturteile vor den Kopf zu stoßen. Metaphern können im Ärztin-Patientin-Gespräch hilfreich sein, um der Patientin Zusammenhänge zu vermitteln, ohne auf Fachwissen zurückgreifen zu müssen. Sie können jedoch durch negative Konnotationen dem Ärztin-Patientin-Verhältnis schaden.

Nicht nur im Gespräch mit der Patientin, auch in der Fachwelt werden Metaphern zum leichteren Verständnis eines komplexen Sachverhalts herangezogen. So wird zum Beispiel das Immunsystem mit Metaphern des Kampfes und der Abwehr beschrieben. Darüber hinaus können Metaphern zum Verständnis neuer Phänomene beitragen, da Informationen von dem bekannten Sachverhalt auf den unbekannten übertragen werden können. Auch dies ist bei der Metapher Medizintourismus der Fall. Für Ärztinnen ist es deshalb in zweifacher Hinsicht von Belang, sich mit Metaphern und den Folgen, die aus ihrer Verwendung resultieren, zu befassen: Zum einen ist es in der Praxis wichtig, im Ärztin-Patientin-Gespräch Metaphern reflektiert einzusetzen, zum anderen ist für die Wissensvermittlung die Wahrnehmung und das Verständnis von Metaphern von Bedeutung.

Trotz ihres innovativen Charakters ist die Verwendung von Metaphern insofern problematisch, als sie häufig einem Bedeutungswandel unterliegen und überdies je nach Kontext normativ verwendet und interpretiert werden können. Dass Auswahl und Verwendung von Metaphern überdacht und gegebenenfalls verändert werden muss, lässt sich am Beispiel der ehemals im medizinischen Kontext üblichen Metapher Mongolismus veranschaulichen. 
Mongolismus war lange Zeit ein gebräuchlicher Ausdruck für Trisomie 21. 1965 stellte jedoch die Mongolei einen informellen Antrag an die WHO, diesen Terminus in Zukunft zu vermeiden. Warum ist der Ausdruck Mongolismus als Bezeichnung für Trisomie 21 problematisch? Er geht auf eine Arbeit des britischen Arztes John L.H. Langdon-Down von 1866 zurück, in der eine ethnische Klassifikation von geistig Behinderten vorgenommen wird (Howard-Jones 1979). Mongolismus ist kein neutraler Ausdruck, obwohl er in deskriptiver Absicht erstmals verwendet wurde. Der Ausdruck illustriert als Metapher eine rein äußerliche Ähnlichkeit zwischen an Trisomie 21 Erkrankten und Mitgliedern der mongolischen Ethnie. Diese Veranschaulichung könnte bei der Adressatin zunächst den Eindruck der Deskription erzeugen. Ein komplexes Krankheitsbild wird dabei jedoch durch seine Benennung auf einzelne Ausprägungen reduziert. Außerdem legt der Ausdruck nahe, eine Einordnung der Krankheit in einen ethnischen Kontext vorzunehmen, die falsch ist. Mongolismus ist in zweifacher Weise normativ: normgebend, indem eine Zuordnung von Krankheit und Ethnie gemacht wird, die schlichtweg falsch ist; und wertend, da er Menschen, die dieser Ethnie angehören, mit behinderten Menschen gleichsetzt. Dieses Beispiel verdeutlicht erstens, dass Sprache normativ ist und zweitens, dass Ausdrücke, die zunächst zutreffend und veranschaulichend erscheinen, nach genauerer Betrachtung neu bewertet und gegebenenfalls bewusst und aktiv aus unserem Sprachgebrauch gestrichen werden müssen.

Metaphern wie Medizintourismus sind im Diskurs dann erfolgreich, wenn sie dazu geeignet sind, bestimmte Aspekte eines bekannten Sachverhaltes in einem unbekannten Phänomen hervorzuheben und dadurch zum Verständnis des neuen Zusammenhanges beitragen. Dass sich der Ausdruck Medizintourismus in den letzten Jahren sowohl in den öffentlichen Medien als auch in Fachdiskursen mehr und mehr durchgesetzt hat, spricht dafür, dass der Ausdruck gut geeignet ist, bestimmte Aspekte des Phänomens zu charakterisieren. Dabei werden Medizintourismus und die davon abgeleiteten Ausdrücke wie Transplantationstourismus oder Reproduktionstourismus unterschiedlich definiert und verwendet. Im weitesten Sinne geht es jedoch bei all diesen Ausdrücken und ihren Interpretationen um Medizin in der globalisierten Welt. Patientinnen gehen für medizinische Behandlungen ins Ausland, gleichzeitig arbeiten Ärztinnen nicht mehr nur im Heimatland.

Dabei wird Tourismus als Metapher zur Beschreibung eines Phänomens herangezogen, das in seiner heutigen Ausprägung viele ethische Probleme aufwirft. Es geht um Autonomie und Vulnerabilität, Verteilungsgerechtigkeit und Kommerzialisierung, Körperbilder und Krankheitsbegriffe, um nur einige Themen zu nennen. Viele Betroffene empfinden die Metapher Tourismus im Zusammenhang mit transnationaler Medizin als unzutreffend, da sie ihre eigene Situation nicht richtig wiedergebe oder bestimmte Aspekte des Phänomens nicht ausreichend erfasse. Es wurde 
bisher noch nicht systematisch, zum Beispiel in einer Textanalyse überprüft, ob diese Vermutungen zutreffen. Bevor jedoch der Ausdruck Medizintourismus im Kontext seiner Verwendung analysiert werden kann, muss zunächst die sprachliche Struktur dieses Neologismus erläutert werden.

\subsection{Die Morphologie ${ }^{3}$ DER MetAPHER MEDIZINTOURISMUS}

Zur Beschreibung neuer Phänomene werden häufig neue Ausdrücke geschaffen. Dabei bietet die Komposition, also die Zusammensetzung mindestens zweier bekannter Wörter, eine wichtige Möglichkeit, Neologismen zu bilden und den Wortschatz zu erweitern. Um ungekannte Sachverhalte zu beschreiben, werden bekannte Ausdrücke als Metaphern herangezogen, um ähnliche Aspekte oder Strukturen in dem neuen Sachverhalt zu benennen oder hervorzuheben (Black 1954). Da es für das Phänomen der transnationalen Medizin, seien es Patientinnen, die für medizinische Behandlungen ins Ausland reisen, Ärztinnen, die im Ausland arbeiten, oder Kliniken, die von ausländischen Trägerinnen betrieben werden, im deutschen Wortschatz keinen Ausdruck gab, wurde in den letzten Jahren das neue Wort Medizintourismus in den Diskurs eingeführt. Eine Komposition, die auf die zwei bekannten Phänomene Medizin und Tourismus zurückgreift.

In der Sprachwissenschaft wird zwischen dem Kopf und dem Kern eines Wortes unterschieden. Während der Kopf die grammatikalischen Eigenschaften eines Wortes bestimmt, wird durch den Kern die Bedeutung festgelegt. Im Deutschen wird der Kopf in der Regel durch das rechts außen stehende Wort einer Komposition bestimmt (Rechtsköpfigkeitsprinzip) (Olsen 1991; Donalies 2002). Bei dem Ausdruck Medizintourismus ist Tourismus der Kopf. Medizintourismus ist ein Determinativkompositum. Das bedeutet, dass ein Bestimmungsverhältnis zwischen Medizin und Tourismus besteht. Determinativkomposita zeichnen sich dadurch aus, dass Kopf und Kern gleich sind. Tourismus ist die zur Erfassung der Strukturen des neuen Phänomens herangezogene Metapher und bestimmt nicht nur die grammatikalischen Eigenschaften des zusammengesetzten Wortes, sondern auch die Bedeutung. Medizin als vorangestellter Baustein dient zur Spezifizierung dieser Bedeutung und ist austauschbar. Dies lässt sich an Ausdrücken wie Reproduktionstourismus oder Transplantationstourismus zeigen: Je nach gewünschtem Grad der Bestimmung des Kernes kann ein engerer Begriff des Wortfeldes Medizin gewählt werden. Präziser wäre dabei die Spezifizierung durch Reproduktionsmedizin, also Reproduktionsmedizintourismus. Dann würde deutlicher, dass es sich um ein Hyponym ${ }^{4}$ zu Medizintourismus handele und nicht

\footnotetext{
${ }^{3}$ Die Morphologie ist ein Teilbereich der Linguistik, der sich mit Aufbau und Struktur von Wörtern beschäftigt.

${ }^{4}$ Die Hyponomie beschreibt die semantische Relation der Unterordnung eines Begriffs zu einem anderen. So verhält sich zum Beispiel Apfel hyponym zu Frucht. Das Gegenwort ist Hyperonomie, es beschreibt die semantische Relation der Überordnung, Frucht verhält sich hyperonym zu Apfel. Jeder Apfel ist eine
} 
etwa um eine Analogie zu Sextourismus. Auf diese besondere Problematik wird in der Diskussion noch näher eingegangen.

Da in dieser Arbeit auf deutsche und englische Texte Bezug genommen wird, soll im Folgenden auch auf die Morphologie von medical tourism eingegangen werden: Dieser Ausdruck wird nicht durch Komposition zweier Nomina, sondern durch die Kombination eines Nomens mit einem Adjektiv gebildet. Das Adjektiv medical dient der Spezifizierung des Nomens tourism, und kann zum Beispiel durch reproductive ersetzt werden. Auch im englischen Ausdruck bildet tourism den Kopf und Kern des Terminus medical tourism.

Bisher liegt keine Untersuchung darüber vor, wie der Ausdruck medical tourism in der ethischen Debatte verwendet wird. Es ist offen, ob und wie der Ausdruck definiert wird. Auch gibt es keine Untersuchungen dazu, welche Denotationen (Grundbedeutungen) und Konnotationen (Nebenbedeutungen) eine Rolle spielen und schon gar nicht darüber, wie diese die Diskussion beeinflussen.

\subsection{Der AnWENDUngSRAHMEn DES TERminus}

Eine orientierende Recherche im Internet, in wissenschaftlichen Publikationen und in den öffentlichen Medien ergab, dass der Ausdruck Medizintourismus in folgenden Bereichen verwendet wird: in der Bioethik, im Journalismus, im Wirtschafts- und Versicherungswesen sowie in der juristischen und in der medizinischen Fachdebatte.

Dieser breite Anwendungsrahmen verdeutlicht den Erfolg der Metapher, das heißt, sie scheint gut geeignet zu sein, um bestimmte Strukturen in dem neuen Phänomen fächerübergreifend zu charakterisieren und dadurch „verstehbar zu machen“ (Lakoff und Johnson 2004, S. 47).

Die Bandbreite des Anwendungsrahmens spiegelt sich auch in der ethischen Diskussion wider und macht diesen für eine Analyse besonders interessant. Der ethische Diskurs ist interdisziplinär ausgerichtet und umfasst kulturanthropologische, philosophische, sprachwissenschaftliche, juristische, wirtschaftliche und medizinische Perspektiven. Alle genannten Fachrichtungen sind von diesem vielschichtigen Problem betroffen und tauschen sich über Fachgrenzen hinweg aus. Der Medizinhistoriker und Germanist Robert Jütte betont, dass Sprache und Semantik der „meeting place of various disciplines" seien (Jütte 2001, S. 11). Eine eindeutige Sprache und ein eindeutiges Vokabular sind also gerade im ethischen Diskurs aufgrund dessen Interdisziplinarität von entscheidender Bedeutung für eine fruchtbare Kommunikation.

Frucht, aber nicht jede Frucht ist ein Apfel. In Bezug auf diese Arbeit ist es von Bedeutung, dass Medizintourismus sich hyperonym zu einer Reihe von anderen Begriffen, zum Beispiel Reproduktionstourismus oder Transplantationstourismus verhält. 
Da die Publikationen im Fachdiskurs zumeist auf Englisch erscheinen, bezieht sich diese Arbeit gleichermaßen auf die Ausdrücke Medizintourismus und medical tourism sowie die entsprechenden Hyponyme, also untergeordnete Ausdrücke, die Tourismus oder tourism im Kontext von medizinischen Behandlungen verwenden.

\subsection{MEDIZINTOURISMUS: EXPLIKATIONEN AUS DER FACHLICHEN DISKUSSION}

Die Vielfältigkeit und Komplexität des Phänomens, welches sich hinter dem Ausdruck Medizintourismus verbirgt, kann beispielhaft anhand unterschiedlicher Explikationen, die sich in der ethischen Debatte finden, verdeutlicht werden. Im untersuchten Textmaterial finden sich sowohl explizite Definitionen von Medizintourismus sowie einige seiner Hyponyme als auch allgemeine Erläuterungen, worauf sich die jeweilige Autorin bezieht. Da die meisten Autorinnen nicht explizit den Anspruch erheben, eine Definition in engerem Sinne zu geben, aber dennoch erläutern, worauf sie sich mit dem Ausdruck Medizintourismus beziehen, verwende ich im weiteren Text den Ausdruck Explikation in Bezug auf Passagen, in denen der Ausdruck Medizintourismus präzisiert wird. Die Untersuchung der gegebenen Explikationen hat zum Ziel, den Bedeutungsumfang beziehungsweise den Anwendungsumfang des Ausdrucks Medizintourismus darzustellen und zu analysieren.

Der Jurist und Bioethiker I. Glenn Cohen beschreibt Medizintourismus als "the travel of patients from their home country to another for the primary purpose of seeking medical treatment"(Cohen 2010, S. 11). Er unterscheidet drei Formen von Medizintourismus und geht dabei von der Gesetzeslage im Heimatland der Patientinnen einerseits und im Land der medizinischen Behandlung andererseits aus. So sei „organ sale“ bzw. „organ tourism“ ein gutes Beispiel für die Form von „medical tourism“, in der die gesuchte Behandlung häufig sowohl im Heimat- als auch im Zielland illegal sei. Eine andere Form von „medical tourism“ sei es, wenn die gesuchte Behandlung zwar illegal im Heimatland, jedoch legal im Zielland sei (Cohen 2010). Dies trifft zum Beispiel auf deutsche Paare zu, die in einen Bundesstaat der USA reisen, um ein Kind mit Hilfe einer Leihmutter zu bekommen. Als dritte Form kategorisiert Cohen jene Fälle, in denen die gesuchte Behandlung in beiden Ländern legal sei, zum Beispiel Bypass-Operationen von Herzkranken aus den USA in medizinischen Einrichtungen in Thailand.

Bruce J. Fried und Dean M. Harris, beide im Gesundheitsmanagement tätig, verstehen dagegen unter medical tourism alle Situationen, in denen Patientinnen außerhalb ihres Heimatlandes medizinische Behandlungen bekommen. Hierzu zählen sowohl diejenigen, die gezielt für eine Behandlung ins Ausland reisen, als auch diejenigen, die sich primär aus anderen Gründen im Ausland aufhalten, jedoch ebenfalls eine medizinische Behandlung in Anspruch nehmen. Dafür 
nennen Fried und Harris als Beispiele sowohl „residents of foreign countries [...] [that] are in the U.S. temporarily for tourism, business, education, or family visits“ als auch „legal immigrants“ und „undocumented aliens“ (Fried und Harris 2007, S. 8). Als Gründe der Betroffenen, für medizinische Behandlungen ins Ausland zu gehen, nennen die Autoren finanzielle Vorteile, umfassendere persönliche Betreuung, höhere Diskretion und die Möglichkeit, einen medizinischen Eingriff mit „exotic vacation“ zu verbinden. Außerdem zeigen sie auf, dass sich das Phänomen reisender Patientinnen häufiger zeige, wenn bestimmte Verfahren oder Güter im Heimatland nicht verfügbar seien. Hierzu verweisen die Autoren zum Beispiel auf die Knappheit von Organen, die zu „transplant tourism“ führe: Patientinnen reisten in andere Länder, „to obtain organ transplants" (Fried und Harris 2007, S. 6).

Die Wirtschaftswissenschaftler Michael D. Horowitz und Jeffrey A. Rosensweig grenzen in ihrem Aufsatz „Medical Tourism - Health Care in the Global Economy“ wiederum das „medical tourism model“ als neues Phänomen von einer „traditional form of international medical care“ ab (Horowitz und Rosensweig 2007, S. 24). Unter dieser „traditionellen“ Form der internationalen Medizin verstehen Horowitz und Rosensweig wohlhabende Patientinnen aus weniger entwickelten Ländern, die in höher entwickelte Länder reisen, um fortschrittliche medizinische Versorgung zu bekommen, die im Heimatland nicht möglich wäre. „Medical tourists“ seien hingegen Patientinnen aus höher entwickelten Ländern, die in andere, oft weniger entwickelte Länder, reisen, um dort medizinische Versorgung in Anspruch zu nehmen, die technisch auch im Heimatland möglich wäre. In ihrem Artikel beziehen sich die Autoren überwiegend auf die Situation in den USA. Als Gründe für Reisen aufgrund medizinischer Behandlungen werden finanzielle Vorteile, Wunsch nach Diskretion und kürzere Wartezeiten genannt (Horowitz und Rosensweig 2007).

Die Ärzte und Gesundheitsökonomen Arnold Milstein und Mark Smith unterscheiden in ihrem Artikel „America's new refugees“ zwischen „medical tourists“ und „refugees“. Dabei seien „medical tourists“ Patientinnen auf der Suche nach „low-cost aesthetic enhancement“; sie seien nicht zu verwechseln mit denjenigen, die aus finanzieller Not medizinisch indizierte Operationen im Ausland vornehmen ließen (Milstein und Smith 2006, S. 1637).

Der Bioethiker Guido Pennings definiert Reproduktionstourismus schließlich als das Reisen potentieller Patientinnen von einer medizinischen Einrichtung, Gesetzgebung oder einem Land, in denen eine bestimmte Behandlung nicht verfügbar sei, in eine andere Einrichtung, Gesetzgebung oder ein anderes Land, in denen sie die gewünschte reproduktionsmedizinisch assistierte Behandlung bekommen können. Nach Pennings ist mit reproductive tourism ein Teilbereich von dem als medical tourism bezeichneten Phänomen gemeint (Pennings 2002). Marcia Inhorn, 
Anthropologin, weist darauf hin, dass zudem reisendes medizinisches Personal, reisende Leihmütter und Organspenderinnen und sogar der Import und Export von Keimzellen als „variation of the phenomenon" betrachtet werden sollten (Inhorn und Gürtin 2011, S. 672).

Diese unterschiedlichen Präzisierungen des Ausdrucks Medizintourismus verweisen einerseits auf die Komplexität des Phänomens, andererseits machen sie die fehlende Eindeutigkeit des Ausdrucks deutlich: Während sich Reproduktionstourismus unter anderem auf ein legales Ausweichen von Patientinnen auf Länder mit anderen Gesetzgebungen bezieht, verbirgt sich hinter Transplantationstourismus oft illegaler Organhandel. Während die einen unter Medizintouristinnen ausschließlich diejenigen verstehen, die für eine medizinische Behandlung ins Ausland reisen, schließen andere Ärztinnen, die im Ausland arbeiten, mit ein. Reproduktions- und Transplantationstourismus sind zudem nur zwei Beispiele für Hyponyme, die sich aus dem Ausdruck Medizintourismus ergeben. Weitere, als synonym verstandene Ausdrücke, zum Beispiel fertility tourism und procreative tourism für reproductive tourism oder organ tourism für transplant tourism, bringen neue Bedeutungsmöglichkeiten mit sich. Dental tourism, stem-cell tourism und sogar death tourism komplizieren das Bild. An der Bandbreite der Bedeutungen lässt sich erkennen, warum die undifferenzierte Verwendung von Medizintourismus problematisch ist. Dies hat Fehlinterpretationen zur Folge, die zur Diskriminierung von Patientinnen bis hin zur Verharmlosung krimineller Handlungen führen.

Für die ethische Debatte ist neben der Uneindeutigkeit des Ausdrucks vor allem problematisch, dass viele Diskursteilnehmerinnen Tourismus im Zusammenhang mit medizinischen Behandlungen verwenden, ohne explizit darzustellen, auf welche Aspekte des Phänomens sie sich beziehen oder an welcher Definition sie sich orientieren. Auch ich verzichte an dieser Stelle auf eine eindeutige Definition, da es Teil dieser Arbeit ist, herauszuarbeiten, in wie vielen unterschiedlichen Zusammenhängen Medizintourismus oder eines seiner Hyponyme Anwendung finden. Obwohl vereinzelt kritische Stimmen fordern, die Tourismus-Metapher im Zusammenhang mit medizinischen Behandlungen nicht mehr zu verwenden, hat eine wissenschaftliche Überprüfung des uneindeutigen Gebrauchs der Metapher bisher nicht stattgefunden.

\subsection{KRITISCHE STIMMEN AUS DEM DISKURS}

Einige Diskursteilnehmerinnen kritisieren explizit einzelne Aspekte der Verwendung von Medizintourismus beziehungsweise der dazugehörigen Hyponyme. Diese Kritik bezieht sich zumeist auf den Ausdruck Reproduktionstourismus.

So fordern zum Beispiel Inhorn und Patrizio, „to rethink the language of 'reproductive tourism', replacing it with more accurate and patient-centered terms“. In einer Studie mit Patientinnen 
und Patienten konnten die Autorinnen zeigen, dass die Betroffenen den Ausdruck als „cavalier and insensitive“ wahrnehmen, da die eigene Reise meist als „highly stressful“ empfunden werde, während der Terminus „fun, leisure, and holidays under the sun“ unterstelle (Inhorn und Patrizio 2009, S. 904-905). Auch Pennings drängt darauf, die „importance of semantics“ zur Kenntnis zu nehmen und stellt, wie auch die Familientherapeutin Petra Thorn, fest, dass Tourismus im medizinischen Kontext mit negativen Konnotationen belegt sei. Die medizinische Behandlung als ursprüngliche Motivation zu der Reise werde in den Hintergrund gedrängt. Dem „fertility tourist" werde unterstellt, zu reisen, um etwas Fremdes und Außergewöhnliches zu erleben (Pennings 2005, S.3572; Thorn 2008). Auch der Präsident der Sociedad Espanola de Fertilidad Roberto Matorras beurteilt den Terminus reproductive tourism als trivialisierend, ungenau und unangemessen, da er zum einen „travelling by pleasure“ unterstelle und zum anderen Assoziationen zu anderen Begriffen wie sex tourism wecke, die als Phänomene nicht vergleichbar seien (Matorras 2005, S. 3571).

In Bezug auf transplant tourism merkt die Anthropologin Yosuke Shimazono an, dass der Ausdruck sowohl die verzweifelten Motive der Patientinnen als auch ethische Fragestellungen außer Acht lasse (Shimazono 2007).

Eine systematische Analyse, die diese Kritikpunkte bestätigt oder widerlegt, liegt bis heute nicht vor. Im Bereich der Reproduktionsmedizin wird seit einiger Zeit zunehmend der Ausdruck crossborder reproductive care ( $C B R C$ ), unter anderem nach einer Empfehlung durch die ESHRE ${ }^{5}$ Task Force on Ethics and Law, verwendet (Pennings et al. 2008). Eine ähnlich intensive Diskussion wurde über den Ausdruck Medizintourismus bisher nicht geführt, wie die Anthropologinnen Inhorn und Zeynep Gürtin feststellen:

[S]cholars studying CBRC and scholars studying other forms of medical tourism may have little intellectual
interaction. Indeed, only rarely are CBRC and other forms of medical tourism explicitly compared and they
are usually not studied together. The CBRC scholarly community remains largely in a separate sphere, pub-
lishing mainly in reproductive medicine and public health journals. Second, the acronym 'CBRC' seems oddly
out of sync with the language being used by other medical tourism scholars. CBRC, which stands for 'cross-
border reproductive care', is a relatively new addition to the scholarly lexicon, coined within the past 2 years
to replace the term 'reproductive tourism' which is seen as inaccurate and pejorative. (Inhorn und Gürtin
2011, S. 667)

Es verwundert, dass in der interdisziplinären ethischen Debatte zwar ein Austausch zwischen den Professionen, nicht aber zwischen den unterschiedlichen medizinischen Fachbereichen wie Chirurgie, Reproduktionsmedizin und Transplantationsmedizin stattfindet, jedenfalls nicht im Kontext von reisenden Patientinnen. Im Gegenteil, während in der Reproduktionsmedizin der Ausdruck insgesamt in Frage gestellt wird, bemüht sich die Transplantationsmedizin zum Bei-

\footnotetext{
${ }^{5}$ ESHRE steht für European Society of Human Reproduction and Embrology.
} 
spiel in der Declaration of Istanbul um eine Definition von "transplant tourism", ohne dabei die möglichen Implikationen auf andere Fachbereiche zu berücksichtigen, geschweige denn Bezug auf die Semantik des Ausdrucks Tourismus zu nehmen (The Transplantation Society und International Society of Nephrology 2008). Auf diese Problematik wird in der Diskussion näher eingegangen.

Bisher werden die unterschiedlichen Bereiche des Phänomens reisender Patientinnen zwar innerhalb der Fachrichtungen, aber relativ isoliert voneinander diskutiert. Dies ist naheliegend, da jeder Fachbereich ihm eigene medizinische sowie rechtliche Aspekte berücksichtigen muss. Dennoch gibt es über die Fachbereiche hinaus ähnliche ethische Fragestellungen. Eine besondere Leistung dieser Arbeit ist es, dass die unterschiedlichen Diskurse der verschiedenen Fachbereiche über Patientinnen, die für eine medizinische Behandlung reisen, vergleichend betrachtet werden. Dies ermöglicht eine umfassende Reflexion des Ausdrucks. Darüber hinaus können auch die unterschiedlichen Aspekte des Phänomens erfasst werden. Eine ausführliche Betrachtung des Phänomens und insbesondere der unterschiedlichen ethischen Fragestellungen in den einzelnen medizinischen Spezialdisziplinen ist jedoch nicht Bestandteil dieser Arbeit. Ihr Schwerpunkt ist vielmehr die Analyse der Metapher Medizintourismus in ihrer Anwendung. In der Diskussion wird allerdings ausführlich auf die Wechselwirkungen von Verwendung des Ausdrucks und Gestaltung der Wirklichkeit eingegangen.

\subsection{FRAGESTELLUNGEN}

Aus obigen Ausführungen ergeben sich eine Reihe empirischer sowie ethischer Forschungsfragen. Aus diskursethischer6 Perspektive stellt sich die Frage, inwieweit es für eine sachliche Diskussion sinnvoll und angemessen ist, die Metapher Tourismus im Zusammenhang mit zu medizinischen Zwecken Reisenden zu verwenden. Diese Frage soll unter anderem anhand einer empirischen Untersuchung der tatsächlichen Verwendung der Metapher in der fachlichen Debatte beantwortet werden. In einer anschließenden ethischen Diskussion soll anhand der Ergebnisse dargelegt werden, inwiefern die Metapher Einfluss auf die Inhalte der Diskussion nimmt und ob die Verwendung des Ausdrucks in der ethischen Debatte gerechtfertigt ist.

Die bis hierhin vorgestellten problematischen Aspekte in der Verwendung der Metapher Medizintourismus führen also zu folgenden Kernfragen:

\footnotetext{
${ }^{6}$ Die Diskursethik umfasst solche Theorien, die sich mit der zentralen Fragestellung auseinandersetzen, welche Regeln in einem Diskurs notwendig sind, um zur Einigung über moralische Fragestellungen zu gelangen. Voraussetzung solcher Theorien ist die Annahme, dass der argumentative Austausch in einem Diskurs am besten dazu geeignet ist, um moralische Regeln zu ermitteln und aufzustellen. Dabei werden Tatsachenaussagen (präskriptive Sätze) im Diskurs gemeinsam argumentativ überprüft.
} 
- Werden durch die Verwendung des Ausdrucks Medizintourismus sowie seiner Hyponyme implizit normative Botschaften vermittelt?

- Welche Folgen ergeben sich aus dem Gebrauch dieser Metaphern für das Verständnis und die ethische Bewertung des Phänomens?

Der ersten der beiden Kernfragen wird mit Hilfe der empirischen Analyse von Texten aus der ethischen Debatte auf den Grund gegangen. Die Ergebnisse daraus werden in der daran anschließenden ethischen Analyse diskutiert. Eine wichtige Voraussetzung sowohl für die empirische Analyse als auch für die ethische Diskussion ist das Wissen und Verständnis um die Wirkungsweisen einer Metapher.

Deshalb werden in Kapitel 3 zunächst Grundlagen zum Metaphernbegriff erläutert. Besonders ausführlich wird dabei auf die Metapherntheorie von Lászlo Kovács eingegangen, da er diese speziell für die Analyse von Metaphern in medizinischen Kontexten entwickelt hat und sie sich also besonders gut für die Analyse der Metapher Medizintourismus eignet. Bei einer Metaphernanalyse stellt der Bezug auf die Grundbedeutungen eines Wortes einen wichtigen Mechanismus für die normativen Wirkungsweisen einer Metapher. Deshalb wird in diesem Kapitel auch die Semantik von Tourismus erarbeitet.

Anschließend erfolgt in Kapitel 4 die empirische Analyse von Texten aus dem ethischen Diskurs. Ein Schwerpunkt liegt dabei darauf, herauszufinden, in welchen konkreten Kontexten die Metapher zur Anwendung kommt. In dieser Arbeit wird die Hypothese einer uneindeutigen Verwendung von Medizintourismus systematisch überprüft. Dazu werden die Texte zum einen auf unterschiedliche Komposita mit dem Kopf Tourismus untersucht, zum anderen werden Explikationen dieser Ausdrücke herausgearbeitet und auf ihre inhaltlichen Aussagen überprüft und miteinander verglichen. So wird ermittelt, was die Autorinnen unter dem Ausdruck Medizintourismus verstehen und auf welche Themengebiete sich die Metapher erstreckt.

Im interdisziplinären ethischen Diskurs prägen unterschiedliche Teilnehmerinnen aus verschiedenen Fachbereichen die Diskussion unter der Verwendung des Ausdrucks Medizintourismus. Deshalb wird untersucht, inwiefern der fachliche Hintergrund der Autorinnen das Vokabular ihrer Arbeit und damit auch die Konzeption des Phänomens beeinflussen. Die systematische Analyse der Texte zeigt dabei, ob sich die Autorinnen auf die semantischen Hauptaspekte von Tourismus beziehen und welche impliziten Werturteile sie damit gegebenenfalls zum Ausdruck bringen. 
In der fachlichen Diskussion wird unter anderem die normative Wirkung der Metapher Medizintourismus problematisiert. Im 5. Kapitel wird aufgezeigt, welche Ziele die Autorinnen verfolgen, die die Metapher verwenden. Denn eine Metapher dient immer dazu, bestimmte Analogien zweier Phänomene hervorzuheben. Dies bedeutet aber gleichzeitig, dass andere Aspekte ignoriert werden. Es wird untersucht, welche Aspekte über die Semantik von Tourismus hinaus in der transnationalen Medizin eine Rolle spielen und ob sie im Verhältnis weniger berücksichtigt werden. Werden tatsächlich Inhalte, die nicht der Semantik von Tourismus entsprechen, ignoriert? Zur Beantwortung dieser Fragen wird die Metapherntheorie von Kovács aufgegriffen. Dabei wird überprüft, inwiefern sich seine Annahmen auf die Verwendung des Ausdrucks Medizintourismus anwenden lassen.

Die normative Verwendung der Tourismus-Metapher wurde bisher vor allem im Zusammenhang mit Reisen aus reproduktionsmedizinischen Gründen kritisiert. Die Kritikerinnen des Ausdrucks unterstellen dabei, dass sich die Verwenderinnen des Begriffs Reproduktionstourismus auf die Freizeitsemantik von Tourismus beziehen und daraus Schlussfolgerungen für die Lebenswirklichkeit der Patientinnen ableiten. Es liegt bis heute keine gründliche Untersuchung vor, die diese normative Verwendung bestätigt. Anhand der Ergebnisse aus der empirischen Untersuchung von Texten aus der ethischen Debatte wird im 6. Kapitel die normative Verwendung sowie die Kritik daran überprüft und einer sorgfältigen ethischen Analyse unterzogen. Aus diskursethischer Perspektive wird überprüft, ob der Ausdruck Medizintourismus der sachlichen Diskussion in einem interdisziplinären Diskurs angemessen ist.

In dieser Arbeit wird die normative Verwendung der Metapher Medizintourismus herausgearbeitet und hinterfragt. Ein besonderes Merkmal stellt dabei die vergleichende und umfassende Analyse der Metapher unter Berücksichtigung der Hyponyme und der dazugehörigen Fachdiskussionen dar. Denn obgleich das als Medizintourismus bezeichnete Phänomen in vielen verschiedenen Fachrichtungen zu beobachten ist, spielt sich die ethische Diskussion bisher überwiegend innerhalb der Fachdisziplinen ab. Aus der Verwendung derselben Metapher in diesen unterschiedlichen Fachbereichen ergeben sich jedoch weitreichende Konsequenzen für den Bedeutungsumfang des Ausdrucks Medizintourismus. Inwiefern dies auch unser Verständnis und damit die Diskussion über das Phänomen von zu medizinischen Zwecken Reisenden beeinflusst, wird in Kapitel 6.2 problematisiert. Im Anschluss daran werden im 7. Kapitel die Kritik aus dem Diskurs reflektiert und alternative Vorschläge diskutiert.

Zusammenfassend werden in der vorliegenden Arbeit die einzelnen Kritikpunkte zur Verwendung von Medizintourismus systematisch herausgearbeitet und überprüft. Dazu wird der Terminus auf seine Denotationen und Konnotationen untersucht. Es wird sowohl die Semantik von 
Tourismus dargestellt als auch der Terminus Medizintourismus im Kontext seiner Verwendung als Metapher analysiert. Auf diese Weise werden die deskriptiven und normativen Aussagen des Metaphernsystems Medizintourismus herausgearbeitet. Die Eignung des Ausdrucks Medizintourismus als Hyperonym, also als übergeordneter Ausdruck dieses komplexen Phänomens wird hinterfragt, und es werden Überlegungen vorgenommen, welche Anforderungen ein geeigneter alternativer Ausdruck erfüllen sollte.

Ziel dieser Arbeit ist es, aufgrund einer systematischen Analyse den Ausdruck neu zu bewerten und gegebenenfalls Alternativen aufzuzeigen. Damit leistet diese Arbeit einen Beitrag dazu, die ethische und gesellschaftliche Diskussion eines weit verbreiteten und umstrittenen Phänomens fundierter zu gestalten. 


\section{Material Und Methoden}

Im Mittelpunkt dieser Arbeit steht der Terminus Medizintourismus als Metapher. Zu seiner Analyse werden verschiedene Theorien aus dem linguistischen, philosophischen und medizinischen Diskurs herangezogen.

Die Beziehung zwischen dem ursprünglichen Begriff eines Ausdruck und einem neuen, mit Hilfe einer Metapher beschriebenen Zusammenhang lässt sich anhand der Interaktionstheorie, die von dem Philosophen und Literaturkritiker Ivor A. Richards begründet und von dem Philosophen Max Black weiterentwickelt wurde, besonders gut zeigen. Diese werden im 3. Kapitel ausführlich vorgestellt. Außerdem werde ich mich auf die Arbeit des Bioethikers László Kovács beziehen, der 2008 in seiner Dissertation verschiedene Metaphern in der Humangenetik analysiert hat. Die Metapherntheorien liefern die theoretische Grundlage sowohl für den Aufbau und die Durchführung der empirischen Untersuchung als auch für die ethische Analyse. Da sich eine Metapher nicht ohne Bezug auf die Bedeutung des Grundbegriffes, in diesem Fall Tourismus, betrachten lässt, wird die Semantik des Ausdrucks Tourismus herausgearbeitet. Dazu werden verschiedene Definitionen herangezogen und vergleichend betrachtet. Unter Berücksichtigung dieser sprachtheoretischen Grundlagen werden Texte aus der medizinethischen Debatte auf die Verwendung des Ausdrucks Medizintourismus hin empirisch untersucht und ausgewertet.

Als Untersuchungsmaterial dient ein Textkorpus von 45 Texten aus der ethischen Diskussion, das in einer systematischen Literaturrecherche ermittelt wurde. Alle diese Artikel verwenden den Ausdruck Medizintourismus oder eines seiner Hyponyme. Das detaillierte Vorgehen bei der Recherche sowie die Ergebnisse werden ausführlich in Kapitel 4.1 vorgestellt. Die empirische Textanalyse wurde mit Hilfe der Software ATLAS.ti durchgeführt. Hierbei handelt es sich um eine Software zur qualitativen Datenanalyse, die also genau dafür entwickelt wurde, eine große Textmenge systematisch zu analysieren. Die Textanalyse zeigt, in welchem Maße auf die Semantik von Tourismus zurückgegriffen wird. Dazu wurden zum Beispiel alle Texte auf die Verwendung bestimmter Vokabeln hin untersucht, wofür die Software ein geeignetes Hilfsinstrument war. Weitere Möglichkeiten des Programms und das genaue Vorgehen der Textanalyse werden im 4. Kapitel dargestellt.

Mit der empirischen Untersuchung wird die Hypothese überprüft, dass die Tourismus-Metapher auf rein sprachlichem Wege die Diskussion über das Phänomen reisender Patientinnen beeinflusst, ja sogar lenkt. In der daran anschließenden ethischen Analyse werden daraufhin die so 
ermittelten Wirkungsweisen der Metapher kritisch hinterfragt und ihre Konsequenzen für die ethische Diskussion problematisiert. In dieser Verzahnung von empirischer Untersuchung und ethischer Analyse liegt die besondere Leistung dieser Arbeit. 


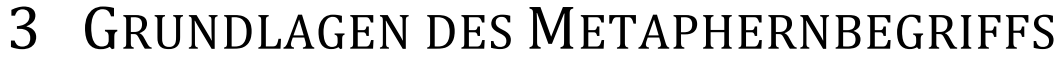

\subsection{VON IVOR RICHARDS ZU MAX BLACK}

Im Brockhaus wird Metapher als „ein sprachliches Ausdrucksmittel“ definiert, mit dessen Hilfe „das eigentlich gemeinte Wort [...] durch ein anderes, das eine sachliche oder gedankliche Ähnlichkeit aufweist“, ersetzt wird. „Die Sprache springt dabei [...] von einem Vorstellungsbereich in den anderen“ (Brockhaus 2009). Sowohl in der Philosophie als auch in den Sprachwissenschaften wurden mittlerweile zahlreiche weitaus komplexere Theorien zur Metapher entwickelt und diskutiert. Im Folgenden soll vor allem die Interaktionstheorie des Literaturwissenschaftlers Ivor Richards und des Philosophen Max Black vorgestellt werden, welche von Kovács weiterentwickelt und auf Metaphern in der Humangenetik angewandt wurde. Die Methode kam also bereits im medizinischen Kontext zur Anwendung und stellt somit ein gutes Instrument zur Analyse des Ausdrucks Medizintourismus dar.

Richards legte 1936 eine weite Definition von Metapher vor, indem er alle Vorgänge als metaphorisch definierte, „bei denen wir unsere Wahrnehmung, unser Denken oder unsere Empfindungen über eine Sache in Worten ausdrücken, die eigentlich zu etwas anderem gehören“. Richards war sich bewusst, dass er mit dieser offenen Definition die Gefahr in Kauf nahm, nicht immer unterscheiden zu können, „ob ein Wort nun wörtlich oder metaphorisch gebraucht [werde]. [...] Ein Wort kann simultan sowohl wörtlich als auch metaphorisch sein“ (Richards 1936, S. 42-43).

Black entwickelte 1954 zwei Theorien zur Metapher: die Substitutionstheorie und die Interaktionstheorie. Laut der Substitutionstheorie handelt es sich um eine Metapher, wenn eine Produzentin ${ }^{7}$ ein Wort benutzt, das in dem benutzten Rahmen nicht wörtlich zu verstehen ist, aber durch ein anderes, wörtlich gemeintes Wort ersetzt werden kann. Die Metapher substituiere also ein anderes Wort. Es sei Aufgabe der Rezipientin, den Bruch zwischen „Rahmen“ und „Fokus“ (des als Metapher verwendeten Wortes) zu erkennen und dann die Metapher aufgrund ihrer inhaltlichen Analogien zu dem eigentlich Gemeinten zu interpretieren. Dies könne nur gelingen, wenn die Metaphernproduzentin sich dabei auf ein allgemeines „System miteinander assoziierter Gemeinplätze“ beziehe (Black 1954, S. 57-58; S. 70-71). So wird sich die Produzen-

\footnotetext{
${ }^{7}$ Black verwendet in seinem Text die Begriffe Sprecher und Leser. Ich verwende in meinem Text stattdessen die Ausdrücke Produzentin beziehungsweise Senderin und Rezipientin oder Adressatin. Dadurch wird deutlich, dass sich die Theorie sowohl auf gesprochene als auch auf geschriebene Kommunikation bezieht.
} 
tin der Metapher „Der Mensch ist ein Wolf“ darauf verlassen, dass Wolf bei vielen Adressatinnen als ein „Verweis auf etwas Wildes, Raubtierhaftes, Verräterisches" verstanden werde (Black 1954, S. 69). An diesem Beispiel wird deutlich, dass die Wirkung einer Metapher nicht auf Wahrheiten beruht, sondern vielmehr auf der Einigkeit über die Assoziationen, die ein bestimmtes Wort hervorruft. Die Senderin muss darauf vertrauen, dass die Metapher in dem Sinne verstanden wird, wie sie sie verwenden möchte. Dabei baut sie auf Gemeinplätze. Im Falle des Wolfes nennt Black „räudig“, „böse“ und „verräterisch“ als Gemeinplatz, allerdings ohne dies zu begründen. Genauso gut könnte die Produzentin sagen wollen, der Mensch sei haarig und fräße Fleisch. Trotzdem kann die durchschnittliche Rezipientin Blacks vorgeschlagene Gemeinplätze vermutlich nachvollziehen. Die Metapher „Wolf" bezieht sich dabei auf ein in der Gesellschaft kulturell verankertes Bild des Wolfes. Die Produzentin verlässt sich darauf, dass der Rezipientin dieses zum Beispiel durch Märchen vermittelte Bild bekannt ist.

Der Substitutionstheorie zufolge kann die Produzentin, anstatt die Metapher „Wolf" zu verwenden, explizit die Eigenschaften des Menschen aufzählen, die sie hervorheben möchte. Es gebe jedoch auch den Fall, dass eine Metapher benutzt werde, um eine Lücke im Wortschatz zu schließen. Dies ist gegeben, wenn eine neue Situation oder ein neues Phänomen benannt werden soll. Analogien zwischen dem bekannten und dem neuen System begründen dabei die Wahl einer bestimmten Metapher (Black 1954).

Mit seiner Interaktionstheorie bezieht Black sich auf Richards, der feststellte, dass „wir beim Gebrauch einer Metapher zwei unterschiedliche Vorstellungen in einen gegenseitigen aktiven Zusammenhang [bringen], unterstützt von einem einzelnen Wort oder einer einzelnen Wendung, deren Bedeutung das Resultat der Interaktion beider ist" (Richards 1936, S. 34). Mit dieser Theorie lassen sich Funktionen und Grenzen einer Metapher beschreiben. Sie besagt, dass durch den Gebrauch einer Metapher zwei zunächst voneinander unabhängige Systeme zueinander in Beziehung gesetzt werden und eine Interaktion zwischen Wort und Systemen ausgelöst wird. Durch die Feststellung bestimmter Analogien zweier Systeme mittels einer Metapher komme das neue System zu einer Benennung, während das Wort, das ursprünglich nur eine Bedeutung (die des ersten Systems) hat, eine neue Bedeutung erhalte. So werde durch die Metapher „Der Mensch ist ein Wolf" zunächst der Mensch als räudig, böse, verräterisch dargestellt, zugleich erscheine der Wolf menschlicher. Solle eine Metapher gelingen, müsse die Adressatin laut Black die alte und die neue Bedeutung zugleich wahrnehmen. Gleichzeitig komme es auf Seiten der Rezipientin zu einer Wahrnehmung des neuen Systems über die Strukturen, die die Metapher durch ihre ursprüngliche Bedeutung vorgebe. Das neue System werde also, metaphorisch gesprochen, durch einen „Filter“ wahrgenommen/erkannt (Black 1954). Dies bedeutet, dass die in 
dem neuen Phänomen erkennbaren Strukturen auf das „Implikationsmuster“ beschränkt sind, welches durch die wörtlichen Verwendungen des Wortes, das als Metapher herangezogen werde, vorgegeben ist. Bestimmte Merkmale eines Phänomens werden betont, während andere, für die es in der Metapher keine Analogien gibt, in den Hintergrund geraten (Black 1954). Eine Autorin kann darüber den Begriff, den wir von einem neuen Phänomen bekommen, durch die vorgegebene Metapher lenken. Dadurch komme es zu „Einstellungsänderungen“, die „regelmäßig aus dem Gebrauch metaphorischer Sprache resultieren“ (Black 1954, S. 73). Die Implikation gründe dabei nicht auf Argumentation, sondern auf Konvention. So führe die Metapher „Der Mensch ist ein Wolf" über die Konvention „der Wolf ist ein ,abstoßendes und angsteinflößendes Objekt““(Black 1954, S. 73) zu dem Urteil „Der Mensch ist angsteinflößend“. Black benennt hier das normative Potential von Metaphern: Der Adressatin kann mit Hilfe einer Metapher die Wertung eines Sachverhaltes ohne explizite Argumentation vermittelt werden.

Darüber hinaus führt Black an, dass sich die Bedeutung einer Metapher je nach dem „Rahmen“, in dem sie verwendet wird, verändern könne. Abhängig vom Rahmen werde auf unterschiedliche Inhalte des Ursprungswortes Bezug genommen. Der Rahmen entscheide darüber, ob ein Wort eine Metapher sei oder nicht. Ein und dasselbe Wort, in zwei Kontexten verwendet, ist nicht zwangsläufig ein und dieselbe Metapher. Unterschiede zwischen den Rahmen führen zu Unterschieden in Zusammenspiel von Fokus (dem als Metapher gebrauchten Wort) und Rahmen (Black 1954). So kann die Metapher „Der Mensch ist ein Wolf“ eine andere metaphorische Bedeutung erlangen, wenn sie zum Beispiel in einen Vortrag über das ausgeprägte Sozialverhalten der Wölfe eingebettet ist. Aber nicht nur der Rahmen, auch die Intention der Produzentin sei für das Verständnis einer Metapher von Bedeutung. Beim gesprochenen Wort können Haltung, Tonfall und Betonung Hinweise auf die Intention der Senderin geben (Black 1954). Auch die Sprachwissenschaftler George Lakoff und Mark Johnson heben die Interaktionsfähigkeit einer Metapher hervor und beschreiben die Metapher als ein Mittel, „das unser Konzeptsystem und die von uns vollzogenen Arten von Alltagsaktivitäten strukturiert“. Dabei sei es „vernünftig anzunehmen, dass Worte allein Realität nicht verändern“, dennoch habe eine Metapher das Potential, unsere Realität zu formen: „Veränderungen in unserem Konzeptsystem verändern auch das, was für uns real ist, und nehmen Einfluss darauf, wie wir die Welt wahrnehmen und wie wir nach diesen Wahrnehmungen handeln“ (Lakoff und Johnson 2004, S. 168).

Bis hierhin konnte gezeigt werden, dass zum Verständnis einer Metapher insbesondere die implizite Konvention, der Rahmen und die Intention der Produzentin von Bedeutung sind. Um nun die Gemeinplätze, auf die durch die Metapher Tourismus Bezug genommen wird, zu erarbeiten, werden verschiedene Definitionen von Tourismus herangezogen. In einer Textauswahl werden 
die jeweiligen Rahmen überprüft. Um sich der Intention der Produzentinnen zu nähern, wird der fachliche Hintergrund der Autorinnen berücksichtigt. Dadurch sollen unterschiedliche Intentionen in der Verwendung des Ausdrucks Medizintourismus aufgedeckt werden. Des Weiteren wird in Bezugnahme auf die Interaktionstheorie herausgearbeitet, welche Implikationen des Phänomens durch die Metapher Medizintourismus hervortreten und welche durch die Metapher nicht erfasst und damit nicht erkannt und benannt werden. Da Kovács die Interaktionstheorie auf Metaphern in der Medizin angewandt hat und deshalb sein Ansatz für diese Arbeit besonders geeignet ist, wird dieser im nächsten Kapitel vorgestellt.

\subsection{LÁSZLó KOVÁCS: DIE VERSCHIEDENEN LEISTUNGEN EINER METAPHER}

Kovács hat in seiner Arbeit von 2008, ausgehend von der Interaktionstheorie von Black, verschiedene Leistungen der Metapher herausgearbeitet: die illustrative, die innovative, die konstitutive und die normative Leistung. In seiner Arbeit „Medizin - Macht - Metaphern“ untersucht Kovács in der Humangenetik gebräuchliche Metaphern auf diese verschiedenen Funktionen. Es handelt sich also um eine Methode, die bereits im medizinischen Bereich erprobt ist.

In der Wirkung einer Metapher greifen diese verschiedenen Leistungen ineinander und sind nicht scharf voneinander abzugrenzen. Zur Erleichterung des Verständnisses werden sie im Folgenden zunächst einzeln besprochen und dann miteinander in Verbindung gesetzt.

\subsubsection{DIE ILLUSTRATIVE LEISTUNG}

Die illustrative Leistung einer Metapher basiert auf Ähnlichkeiten zwischen zwei Sachverhalten. Die Metapher verdeutlicht einen Zusammenhang oder ein Phänomen und erleichtert dessen Verständnis, indem sie einen anderen Sachverhalt zur Veranschaulichung heranzieht. Eine Metapher kann nur verstanden werden, wenn der Adressatin der in der Metapher verwendete alte Sachverhalt bekannt ist. Hier stimmt Kovács mit Black überein, der die Gemeinplätze als Grundlage für das Verständnis einer Metapher beschreibt. Unter Berücksichtigung der antizipierten Gemeinplätze der Adressatinnen kann die Botschaft einer Metapher je nach Kontext geplant werden. Das bedeutet jedoch auch, dass eine Metapher, die den Kontext wechselt, eine Botschaft in sich tragen kann, die zuvor übersehen wurde, beziehungsweise, dass sie für unterschiedliche Adressatinnen unterschiedliche Zusammenhänge illustrieren kann.

Im Rahmen der illustrativen Leistung wird der Adressatin durch die Metapher ein bestimmter Deutungsrahmen vorgeschlagen, in den sie den neuen Sachverhalt einordnen und damit weiterentwickeln kann. 


\subsubsection{DIE INNOVATIVE LEISTUNG}

In dieser Weiterentwicklung des Verständnisses des neuen Sachverhaltes auf der einen Seite und der Kontextabhängigkeit der Bedeutung einer Metapher auf der anderen Seite liegt das Potential für die innovative Leistung einer Metapher. Eine Metapher wird zumeist aufgrund einer bestimmten Ähnlichkeit verwendet. Da „dieselben Metaphern in anderen Diskursen [...] möglicherweise eine ganz andere Funktion [haben], sind [sie] mit ganz anderen ,locating narratives` verbunden und regen die Phantasie in eine ganz andere Richtung an“ (Kovács 2009, S. 42). Die Adressatin wird durch die innovative Leistung dazu angeregt, kreativ nach weiteren als der für sie am deutlichsten ins Auge springenden Parallele zwischen dem alten und dem neuen Kontext zu suchen.

Unterschiedliche Diskursteilnehmende können der Metapher in anderen Kontexten innerhalb ihres Deutungsrahmens eine andere Bedeutung zuweisen: „Diese neu entstandene und nicht berechenbare Bedeutung ist die innovative Leistung der Metapher" (Kovács 2009, S. 57). Das bedeutet, dass jede erst einmal aufgeworfene Metapher potentiell neue und im Vorfeld nicht berechenbare Bedeutungen enthält.

Eine Metapher dient der Bildung von Assoziationen. Dadurch kann sie die Kreativität aller am Diskurs Teilnehmenden fördern, und es kann zu über die von der Produzentin beabsichtigten Assoziationen hinausgehenden Interpretationen kommen. So wird eine Metapher im aktiven Diskurs beständig weiterentwickelt und unterliegt laufend Veränderungen. Neue Teilaspekte, die zunächst nicht in Betracht gezogen wurden, können erkannt und diskutiert werden.

\subsubsection{DiE KONSTITUTIVE LEISTUNG}

Während die innovative Leistung einer Metapher die Kreativität innerhalb des Deutungsrahmens anregt, wird diese durch die konstitutive Leistung beschränkt. Die Methode ist dabei dieselbe: Das Verständnis des neuen Phänomens wird durch den Deutungsrahmen der Metapher geprägt. Während aber die innovative Leistung die Suche nach weiteren Parallelen beschreibt (Anregung der Kreativität), kennzeichnet die konstitutive Leistung die Festschreibung der so gefundenen Parallelen (Einschränkung der Kreativität). Die konstitutive Funktion einer Metapher beschreibt die Leistung, einen Sachverhalt auf die im Deutungsrahmen festgelegte Art und Weise zu verstehen. Das bedeutet, dass neue Teilaspekte stets zunächst im Sinne der vorliegenden Leitmetapher interpretiert werden, sofern der Deutungsrahmen dies zulässt (Kovács 2009). Die konstitutive Leistung einer Metapher beschreibt die Möglichkeit der Konstruktion eines „Metaphernsystems“, in dem aufeinander einwirkende Komponenten mit zueinander passenden Metaphern benannt werden. Das bedeutet, dass über die Metapher hinaus Vokabular aus dem 
Wortfeld der Leitmetapher auf den neuen Sachverhalt übertragen wird. Jedes dieser dem Wortfeld der Leitmetapher entlehnten Wörter fungiert nun wiederum als Metapher mit allen Funktionen.

Die konstitutive Leistung beschreibt die selektive Betrachtung der Thematik durch die Metapher. Ähnlichkeiten werden durch den Gebrauch von Metaphern hervorgehoben, da die Nutzerin einer Metapher auf bestimmte Punkte hinweisen möchte, andere Aspekte eines Sachverhaltes werden jedoch übersehen oder bewusst außen vor gelassen: „Metaphern bilden ein System und in diesem System heben sie bestimmte Aspekte hervor und verdecken andere, da die Bedeutungsfelder metaphorischer Konzepte mit dem zu Beschreibenden nicht deckungsgleich sind“ (Kovács 2009, S. 38).

Die innovative und konstitutive Leistung können als Gegenspielerinnen verstanden werden: Wenn auf der einen Seite Metaphern Sachverhalte in alte Denkstrukturen pressen und auf diese beschränken können, kann auf der anderen Seite eine in einem bereits bestehenden Diskurs neu aufgeworfene Metapher das Gegenteil bewirken. So können durch eine Metapher im Vorfeld festgefahrene Strukturen aufgebrochen und die Rezipientin zu neuen Denkvorgängen angeregt werden (Kovács 2009). Auch der Übergang von der konstitutiven zur normativen Funktion ist fließend. Sobald nur Teilaspekte berücksichtigt werden, wie dies durch die konstitutive Leistung der Fall ist, liegt Normativität vor, da bestimmte Aspekte zu Lasten anderer gewichtet werden.

\subsubsection{DIE NORMATIVE LEISTUNG}

Etabliert sich eine Metapher im Diskurs, so kann aus der konstitutiven Leistung eine normative werden. Der neue Sachverhalt ist dann auf die Metapher festgelegt und es kommt zur „Normierung einer Sichtweise [...], die nicht in der Sache, sondern nur in der Metapher begründet ist“ (Kovács 2009, S. 61).

Kann eine Metapher durch ihre illustrative Leistung zum Verständnis des neuen Zusammenhanges beitragen, trägt sie Überzeugungskraft. Diese Überzeugungskraft ist jedoch wahrheitsunabhängig, sie basiert allein auf der Veranschaulichung, bzw. auf der Übereinstimmung der Gemeinplätze, wie auch Black mit dem Beispiel „Der Mensch als Wolf" deutlich macht. Dabei kann die bloße Wiederholung der Metapher die Wahrnehmung des neuen Sachverhaltes kanalisieren: „Wenn ein Diskurs immer dieselbe Metapher verwendet, trägt sie dadurch zur Normierung einer Sichtweise bei, die nicht in der Sache, sondern nur in der Metapher begründet ist. [...] Die Wahl des Sprachbildes ist ein normativer Akt, der einen Einfluss auf die Wahrnehmung hat" (Kovács 2009, S. 61). 
Ihre normative Wirkkraft kann eine Metapher nur dann entfalten, wenn sie von der Rezipientin entsprechend interpretiert wird. Metaphern treffen indirekte Aussagen; die Produzentin verlässt sich auf die illustrative Überzeugungskraft der Metapher, anstatt explizit ihren Standpunkt zu erläutern. Dabei kann es zu Missverständnissen bzw. Fehlinterpretationen kommen, beispielsweise dann, wenn die Adressatin eine andere Ähnlichkeit zwischen altem und neuem Phänomen stärker gewichtet als die Senderin. Die Gefahr besteht insbesondere dann, wenn Rezipientin und Produzentin verschiedenen Rahmen angehören, zum Beispiel unterschiedlichen Expertinnenkreisen, oder wenn aus anderen Gründen keine Gemeinplätze vorliegen. Dazu kommt, dass eine Metapher keinen Wahrheitsanspruch in sich birgt, das heißt, eine Autorin kann bewusst Ähnlichkeiten unterstellen und durch die Überzeugungskraft der Metapher hervorheben (Kovács 2009). Aufgezeigte Ähnlichkeiten müssen also von der Adressatin immer kritisch hinterfragt werden.

In der innovativen Funktion liegt ein weiteres Potential zur normativen Funktion: Metaphern schlagen denkbare Ähnlichkeiten vor, die dann in die Realität umgesetzt werden. Black schildert diesen Vorgang in seiner Interaktionstheorie, Kovács schreibt in diesem Zusammenhang, dass Metaphern nicht nur „bereits existierende Ähnlichkeiten aufzeigen, sondern sie erst schaffen“ (Kovács 2009, S. 36). Eine Metapher strukturiert unsere Vorstellung von einem Phänomen. Als Rezipientinnen werden wir versuchen, Leerstellen des Metaphernsystems mit Inhalt zu füllen. So wird auch aus der konstitutiven eine normative Funktion: Eine Metapher ist besonders überzeugend, wenn verschiedene aus dem Wortfeld stammende Metaphern ebenfalls auf den Sachverhalt übertragen werden können. Es kommt zu einer Normierung der Sichtweise auf das Phänomen, die jedoch das neue Phänomen nur partiell abbilden kann.

Die von Kovács beschriebenen Leistungen greifen derart ineinander, dass ich es für sinnvoller halte, die illustrative, innovative und die konstitutive Leistung zu differenzieren, wobei jede dieser Funktionen normatives Potential in sich trägt: Die illustrative Leistung führt zur bildhaften Hervorhebung bestimmter Aspekte, immer zu Lasten anderer, nicht erkannter Aspekte, und umfasst eine implizite, wahrheitsungebundene Überzeugungskraft. Die innovative Leistung ermöglicht die Erfassung zunächst nicht beachteter Parallelen, kann aber auch zu deren Konstruktion beitragen. Die konstitutive Funktion begrenzt die Wahrnehmung des Phänomens auf einen bestimmten, vorgegebenen Deutungsrahmen. In diesem Sinne ist die Normativität einer Metapher zunächst epistemisch, das heißt sie normiert die Erkenntnis bestimmter Strukturen eines neuen Sachverhaltes: „Die Metapher bestimmt, welche Fragen gestellt werden sollen und welche nicht als relevant zu sehen sind“ (Kovács 2009, S. 61). Darüber hinaus können über die Funktionen einer Metapher moralische Wertungen von dem alten auf den neuen Sachverhalt übertragen 
werden, die allein über die Metapher zum Ausdruck gebracht und für die nicht explizit argumentiert werden muss. Dies entspricht einer moralischen Normativität.

Im Folgenden werden zum besseren Verständnis die unterschiedlichen Leistungen einer Metapher an einem Beispiel erläutert. Dazu wird die bekannte und in der Medizin bedeutende Metapher „Der Mensch als Maschine“ herangezogen.

\subsubsection{VERDEUTLICHUNG DER LEISTUNGEN EINER METAPHER ANHAND DES BEISPIELS „DER MENSCH ALS MASCHINE"}

Die zu Beginn des 20. Jahrhunderts populäre Metapher „Der Mensch als Maschine“ veranschaulicht, dass es sich bei dem Körper des Menschen um ein aus einzelnen Teilen zusammengesetztes Ganzes handelt (illustrative Funktion). Die Vorstellung der einzelnen Teile als mechanische Einheiten ermöglicht die Entdeckung mechanischer Eigenschaften der einzelnen Organsysteme. So kann das Herz zum Beispiel als Pumpe identifiziert und diskutiert werden (innovative Funktion). Die Metapher vermag es jedoch nicht, seelische Zustände zu erfassen, da Maschinen keine seelischen Zustände aufweisen (konstitutive Funktion). Die Metapher „Der Mensch als Maschine" hebt den Körper im Gegensatz zur Psyche oder Seele hervor, bewirkt also ein körperbetontes Menschenbild (epistemische Normativität). Dies hat weitreichende Auswirkungen, so wird der Körper, losgelöst von der Seele, als bedenkenlos körperlichen Eingriffen und anderen Manipulationen durch den Menschen zugänglich dargestellt (moralische Normativität).

$\mathrm{Zu}$ untersuchen ist, ob der Ausdruck Medizintourismus auf vergleichbare Weise eine Veranschaulichung des Phänomens reisender Patientinnen bewirkt. Die Illustration könnte sich jedoch auf wenige Teilaspekte im Gesamtphänomen beschränken. Aus einer einseitigen Veranschaulichung würde dann eine normative Bewertung des Phänomens und aller Beteiligten folgen, die problematisch und gegebenenfalls sogar diskriminierend sein kann. Solche Überlegungen lassen sich sinnvoll und gezielt mit Kovács Ansatz, eine Metapher auf verschiedene Funktionen zu prüfen, untersuchen.

Zusammenfassend lässt sich Kovács zufolge feststellen, dass Metaphern

einerseits aus der semantischen Perspektive gedeutet werden [können]. Dazu ist unter anderem die Interaktionstheorie Blacks geeignet: Die inhaltliche Botschaft der Metapher basiert nach diesem Deutungsmodell auf dem ,System miteinander assoziierter Gemeinplätze'. Andererseits gehen neuere Theorien davon aus, dass einzelne Metaphern zwar identifiziert werden können, aber es sie fast ausschließlich im Zusammenhang mit einer übergeordneten metaphorischen Gedankenstruktur gibt, einer Leitmetapher. Diese Leitmetaphern haben nicht nur eine illustrative Funktion, sondern sie strukturieren die Wahrnehmung der Welt, sind innovativ, konstitutiv und normativ für die Weltanschauung (Kovács 2009, S. 62-63).

Beide Ansätze werden in dieser Arbeit in der Textanalyse aufgegriffen: Zum einen verlangt die Interaktionstheorie die Berücksichtigung des Deutungsrahmens, also die Berücksichtigung der 
Semantik der vorgeschlagenen Metapher. Deshalb wird im weiteren Verlauf dieser Arbeit die Semantik des Ausdrucks Tourismus vorgestellt, um die Gemeinplätze zu ermitteln. Zum anderen entfaltet eine Metapher ihre unterschiedlichen Funktionen abhängig vom Diskurskontext. In dieser Arbeit liegt dabei der Schwerpunkt auf der Verwendung der Metapher Medizintourismus in der ethischen Diskussion. Um kontextabhängige Interpretationen beziehungsweise Konnotationen ausfindig zu machen, werden sowohl der fachliche Hintergrund der Autorinnen (zum Beispiel Medizin, Philosophie, Anthropologie) als auch die Eingebundenheit des Ausdrucks in die jeweils relevanten medizinischen Bereiche (zum Beispiel Transplantationsmedizin, Reproduktionsmedizin, Chirurgie) untersucht. In der Diskussion werden diese Ergebnisse anhand der von Kovács unterschiedenen Leistungen eingeordnet und analysiert.

\subsection{Die Semantik Des Ausdrucks Tourismus}

Im Folgenden werden die Bedeutungen des Ausdrucks Tourismus unter Berücksichtigung verschiedener Definitionen aus unterschiedlichen Fachbereichen erarbeitet. Dabei wird auf allgemein gültiges lexikales Wissen ebenso verwiesen wie auf Definitionen aus den Fachbereichen Wirtschaft und Anthropologie, die sich mit dem Phänomen Tourismus wissenschaftlich auseinandersetzen. In einem zweiten Schritt wird aufgezeigt, inwiefern sich Inhalte dieser Definitionen in der ethischen Debatte in der Kritik um den Ausdruck Medizintourismus wiederfinden. Dazu werden Beispiele genannt, in denen Diskursteilnehmende Assoziationen zum TourismusBegriff benennen.

Lexikale Definitionen spiegeln die allgemeinen Zusammenhänge wider, in denen ein Wort verwendet wird (Shibles und Maier 2011). Mittels lexikaler Definitionen können wir also im Sinne von Black wichtige Einblicke in Gemeinplätze gewinnen. Im Brockhaus wird „Tourismus“ im engeren Sinn als „verbreitete Freizeitaktivität der Bevölkerung, die mit Erholung und Vergnügen verbunden ist und für die sich eine touristische Dienstleistungswirtschaft herausgebildet hat" definiert. Im weiteren Sinn werden „alle Phänomene, die mit vorübergehendem Ortswechsel von Personen zusammenhängen und mit mindestens einer Übernachtung verbunden sind“ in die Begriffsbestimmung eingeschlossen (Brockhaus 2009). Der Brockhaus bezieht sich auf die World Tourism Organization (WTO), die 1991 eine weite Definition zu wirtschaftsanalytischen Zwecken entwickelt hat. Diese umfasst alle „Aktivitäten von Personen, die an Orte außerhalb ihrer gewohnten Umgebung reisen und sich dort zu Freizeit-, Geschäfts- oder bestimmten anderen Zwecken nicht länger als ein Jahr ohne Unterbrechung aufhalten“ (World Tourism Organization 1995). Zusätzlich führt der Brockhaus an, dass es im alltäglichen Sprachgebrauch zu „einer Gleichsetzung von Urlaub und Tourismus“ komme (Brockhaus 2009). Der Ethnologe Ronald Lutz definiert Tourismus als „Traum des nicht gelebten, aber doch ersehnten Lebens“. Die wäh- 
rend des Urlaubs erlebte Welt stelle sich als Spiel dar, das nach dem Aufenthalt als beendet betrachtet werde. Im Urlaub sollen sich „Träume erfüllen, die ansonsten nur als Versprechung existieren, aber keine realen Chancen zur Verwirklichung besitzen“ (Lutz 1992, S. 247).

Da in dieser Arbeit auch englischsprachige Texte untersucht werden, wird im Folgenden auch auf englischsprachige Definitionen eingegangen, um mögliche sprachgebundene Konnotationen zu erfassen beziehungsweise auszuschließen. Die Definition des Oxford English Dictionary für „tourism“ lautet: „The theory and practice of touring; travelling for pleasure. (Orig. usually depreciatory.) Also, the business of attracting tourists and providing for their accommodation and entertainment; the business of operating tours“. Die WTO-Definition, auf die sich der Brockhaus bezieht, existiert auch in englischer Sprache, sie lautet: „The activities of persons traveling to and staying in places outside their usual environment for not more than one consecutive year for leisure, business and other purposes" (World Tourism Organization 1995). Die Encyclopeadia Britannica definiert „tourism“ als „the act and process of spending time away from home in pursuit of recreation, relaxation, and pleasure, while making use of the commercial provision of services“ (Encyclopaedia Britannica).

Versucht man, die genannten Definitionen auf das Phänomen reisender Patientinnen anzuwenden, stellt man fest, dass einige Definitionen durchaus als passend erachtet werden können, während andere nicht gelten. Dabei kommt es nicht nur auf die einzelne Tourismus-Definition an, sondern auch auf den konkreten Zusammenhang. So ließe sich mit der WTO-Definition jede medizinische Behandlung unter „bestimmten anderen Zwecken“ einordnen, womit alle Reisen, die eine medizinische Behandlung beinhalten, dem Tourismus-Phänomen zugehörig wären. Migrantinnen, die sich schon länger als ein Jahr in dem Land der medizinischen Behandlung aufhalten und eine medizinische Behandlung in Anspruch nehmen, würden durch die WTO-TourismusDefinition nicht erfasst. Dies widerspricht jedoch ausdrücklich der Definition von Medizintourismus durch Fried und Harris, die sowohl „legal immigrants“ als auch „undocumented aliens“ als Medizintouristinnen verstehen (Fried und Harris 2007, S. 8). Lutz' Verständnis von Touristinnen lässt sich nicht schlüssig auf Patientinnen übertragen, die für eine medizinische Behandlung ins Ausland reisen. So wollen zum Beispiel Patientinnen, die zu reproduktionsmedizinischen Zwecken reisen, das ersehnte Leben mit einem Kind Wirklichkeit werden lassen und dieses in erster Linie über die Reise (den Urlaub) hinaus.

Einige Aspekte werden in mehreren Definitionen benannt. Drei Hauptaspekte, im Sinne von Blacks Gemeinplätzen, die allgemein mit Tourismus in Verbindung gebracht werden, können 
aufgezeigt werden: die Aspekte ,Reisen im Sinne von Ortswechsel' ${ }^{1}$, ,Tourismus als Freizeitgestaltung ${ }^{9}$ und ,Tourismus als Dienstleistungszweig mit wirtschaftlichen Interessen ${ }^{10}$. Unterschiedliche Bedeutungen zwischen dem deutschen Wort Tourismus und dem englischen Ausdruck tourism konnten nicht festgestellt werden.

Die drei semantischen Hauptaspekte Ortswechsel, Freizeit und Wirtschaftszweig werden auch in der medizinethischen Debatte von Kritikerinnen des Ausdrucks Medizintourismus als Konnotationen benannt: Pennings stellt fest, dass Tourismus sich meistens auf Reisen zur Erholung beziehe (Pennings 2002). Inhorn interpretiert Tourismus als „leisure travel“ (Inhorn und Patrizio 2009, S. 904) und beruft sich dabei auf die Definition des Rechtswissenschaftlers Richard Storrow, der Tourismus als "type of traveling that involves leisure, pleasure and free time" beschreibt. Storrow erwähnt gleichfalls die wirtschaftlichen Aspekte, indem er eine zweite Definition von "tourism as an industry“ formuliert (Storrow 2005, S. 299).

In einer systematischen Textanalyse soll nun unter Berücksichtigung der Semantik von Tourismus überprüft werden, inwiefern Texte aus dem medizinethischen Diskurs bei der Verwendung des Ausdrucks Medizintourismus auf einen oder mehrere dieser drei Hauptaspekte Bezug nehmen.

\footnotetext{
8 Brockhaus: „,vorübergehender Ortswechsel“, WTO: „an Orte außerhalb ihrer gewohnten Umgebung reisen“ bzw. „persons traveling to and staying in places outside their usual environment", Oxford English Dictionary: "the theory and practice of touring; travelling for pleasure“, Encyclopaedia Britannica: „spending time away from home"

${ }^{9}$ Brockhaus: „verbreitete Freizeitaktivität der Bevölkerung, die mit Erholung und Vergnügen verbunden ist“, WTO: „zu Freizeit-, Geschäfts- oder bestimmten anderen Zwecken“ bzw. „for leisure, business and other purposes“, Encyclopaedia Britannica: „in pursuit of recreation, relaxation, and pleasure“

${ }^{10}$ Brockhaus: „touristische Dienstleistungswirtschaft“, Oxford English Dictionary: „the business of attracting tourists and providing for their accommodation and entertainment; the business of operating tours", Encyclopaedia Britannica: „the commercial provision of services“
} 


\section{EMPIRISCHE ANALYSE}

\subsection{LITERATURSUCHE NACH ZU ANALYSIERENDEN ARTIKELN}

Die Verwendung der Metapher Medizintourismus ist deshalb von Bedeutung, weil damit zugleich normative Aussagen verbunden sein können. Dies könnte sich insbesondere auf die ethische Debatte auswirken und wird deshalb im Folgenden systematisch untersucht. Zu diesem Zweck wurde im Juli 2010 eine erste Literaturrecherche in den Datenbanken BELIT11 und PubMed vorgenommen. Da es sich um einen noch jungen Ausdruck handelt, wurde der Zeitraum der Recherche auf 01.01.2000 bis 20.07.2010, beziehungsweise in einer zweiten Literatursuche bis 10.02.2012 begrenzt. Dabei ergaben sich 45 einschlägige ethische Texte, die systematisch analysiert wurden.

Zunächst ging es darum, möglichst viele Texte, die Tourismus im Zusammenhang mit medizinischen Behandlungen verwenden, zu erfassen. Da in den Datenbanken BELIT und PubMed das Schlagwort medical tourism erst seit 2010 bzw. 2008 vergeben wird, kam eine Schlagwortsuche zur Erfassung des Analysematerials nicht in Frage. Eine Schlagwortsuche mit medical tourism wurde jedoch dazu genutzt, andere Schlagwörter zu bestimmen, um ein umfassendes Rechercheergebnis zu erhalten. So konnte der Anwendungsrahmen auf folgende Bereiche eingegrenzt werden: Medizin, Bioethik, Wirtschaft, Recht und Journalismus.

Um relevante Artikel zu erhalten, wurde zunächst eine Stichwortsuche durchgeführt, die alle Artikel erfasste, die im Titel oder Abstract das Stichwort tourism enthielten. Dieses Ergebnis wurde dann mit MeSH-terms kombiniert, die dem Anwendungsrahmen entsprechen: reproduction, reproductive techniques, economics, delivery of health care, jurisprudence, euthanasia und organ transplantation. Die Auswahl wurde auf Sprache und Relevanz für das Thema geprüft. Am 10.07.2010 wurden insgesamt 102 Artikel (in Deutsch oder Englisch) erfasst, die Tourismus im Zusammenhang mit medizinischen Behandlungen verwenden. Am 10.02.2012 wurde die Literatursuche aktualisiert und auf den Zeitraum bis zum 10.02.2012 erweitert. Insgesamt wurden 291 Artikel erfasst.

\footnotetext{
${ }^{11}$ BELIT ist die Bioethik-Literaturdatenbank des Deutschen Referenzzentrums für Ethik in den Biowissenschaften (drze). Sie enthält deutsche, englische und französische bioethische Publikationen.
} 
Eine Grafik, die die Ergebnisse beider Recherchen unter Berücksichtigung des Erscheinungsjahres darstellt, zeigt, dass der Gebrauch des Ausdrucks in den vergangenen 10 Jahren bis heute deutlich angestiegen ist:

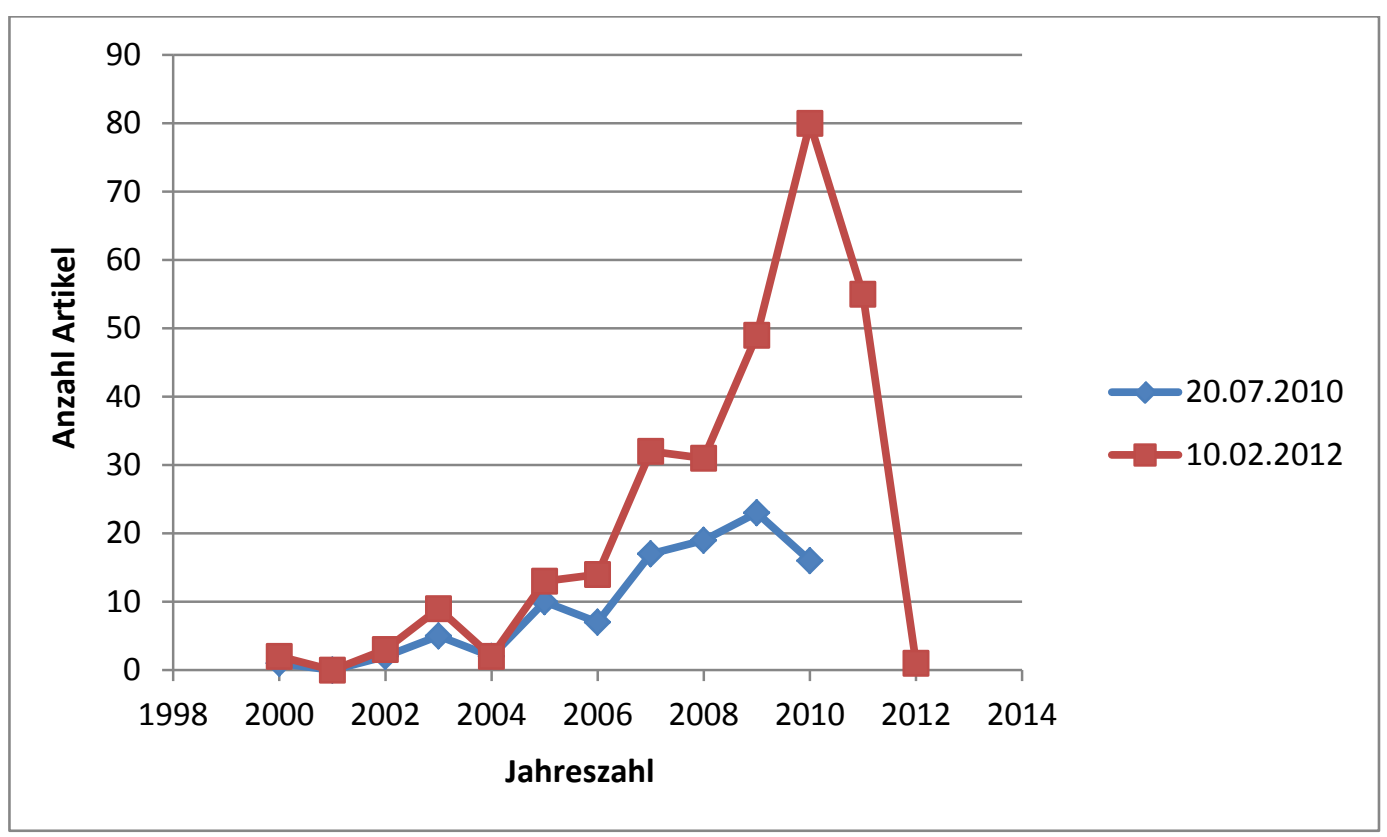

Abb. 1: Anzahl der recherchierten Artikel in Relation zum Veröffentlichungsjahr. Die beiden Kurven bilden die unterschiedlichen Ergebnisse zu zwei verschiedenen Recherchezeitpunkten ab.

Die Grafik stellt die Anzahl der recherchierten Artikel auf der y-Achse in Abhängigkeit von ihrem Erscheinungsjahr auf der x-Achse dar. Die unterschiedlichen Kurven beschreiben den Recherchezeitpunkt.

Der Abfall der blauen Kurve nach 2009 sowie der roten Kurve nach 2010 ist durch den Zeitpunkt der Recherche und die Anzahl der bis dahin in den Datenbanken noch nicht erfassten Artikel zu erklären. Es ist davon auszugehen, dass für den Zeitraum ab 2010 eine deutliche Anzahl weiterer Artikel in die Datenbanken aufgenommen wird. Der Vergleich beider Kurven zum Zeitpunkt 2010 stützt diese Annahme.

\subsection{QUANTITATIVE UND QUALITATIVE ANALYSE MIT ATLAS.TI}

Zur empirischen Auswertung des Textmaterials wurde die Software ATLAS.ti verwendet. ATLAS.ti wurde für die computergestützte qualitative Analyse entwickelt. Darüber hinaus ermöglicht die Software die quantitative Analyse des bearbeiteten Materials. Mit Hilfe des Programms können ausgewählte Textstellen selbst erstellten Kategorien (sogenannten Kodes) zugeordnet werden. Diese Kodes, also Gruppierungen unterschiedlicher Zitate, können in der Auswertung zueinander in Beziehung gesetzt werden. Da die Software gleichermaßen die Erfassung inhaltli- 
cher und formaler Aspekte sowie eine qualitative wie auch quantitative Analyse von Textmaterial ermöglicht, wurde sie zur Bearbeitung der Fragestellung dieser Arbeit herangezogen. Die Analyse wurde in Anlehnung an die NCT-Methode ${ }^{12}$ von Susanne Friese durchgeführt, die diese eigens für die Auswertung von Materialien mit ATLAS.ti entwickelt hat (Friese 2012).

Um die spätere Auswertung zu erleichtern und um einen ersten Zugang zu dem Material zu gewinnen, wurden die ausgewählten Texte einmal gelesen und anhand folgender Fragen unterschiedlichen Kategorien (sogenannten Dokumentenfamilien ${ }^{13}$ ) zugeordnet:

a) Welcher Tourismus-Ausdruck wird verwendet?

b) Wird in dem Text eine Explikation oder Definition des verwendeten Ausdrucks vorgenommen?

c) Welchem Fachbereich gehören die jeweiligen Autorinnen an?

Diese Kategorie hat sechs Dokumentenfamilien: Anthropologie, Ethik/Philosophie, Journalismus, Medizin/Zahnmedizin, Recht, Wirtschaft/Management. Bei Mehrautorenschaften ist es möglich, dass ein Dokument mehreren Dokumentenfamilien der Kategorie fachlicher Hintergrund der Autorinnen zugeordnet ist.

Die Einordnung in diese Dokumentenfamilien ermöglicht eine kontextabhängige Analyse in Bezug auf fachlich bedingte unterschiedliche Interpretationen und Konnotationen der Metapher.

d) In welcher Fachzeitschrift ist der jeweilige Text veröffentlicht?

Diese Einordung ermöglicht eine kontextabhängige Analyse in Bezug auf regional unterschiedliche Interpretationen der Metapher.

Im nächsten Schritt wurden zehn Texte offen kodiert, das heißt Satz für Satz ausgewertet und entsprechenden Kodes zugeordnet (Friese 2012). Einige Kodes wurden anhand der Fragestellungen vorher formuliert, andere kamen während der Auswertung dazu. Die benutzte Kodeliste war zu jedem Zeitpunkt der Auswertung offen für weitere Kodes. Die Kodierungen dienten der Erfassung und Sortierung der in den Texten behandelten Themen.

${ }^{12}$ NCT steht für noticing-collecting-thinking

13 Über die Sortierung aller Artikel in Dokumentenfamilien erfolgte die Erstellung von ausdrucksbezogenen Dokumentenfamilien. Jeder Text wurde auf den oder die verwendeten Tourismusausdrücke untersucht und zu jedem Ausdruck wurde eine Familie erstellt. Jeder weitere Text, der denselben Ausdruck verwendet, wurde dieser Familie ebenfalls zugeordnet. 
Aufgrund der Fragestellungen wurden drei Kodes im Vorfeld erstellt. Sie orientieren sich an den semantischen Aspekten von Tourismus. Um festzustellen, inwieweit die Semantik der Metapher zur Erfassung und Beschreibung des Phänomens herangezogen wird, wurde überprüft, ob die semantischen Felder des Ausdrucks Tourismus in den Texten aufgegriffen und bedient werden. Es wurden die drei Kodes „Semantisches Feld: Wirtschaft“, „Semantisches Feld: Reisetätigkeit/Ortswechsel“ und "Semantisches Feld: Erholung/Freizeit" erstellt. Diese Methode nennt sich halboffenes Kodieren, da einige Kodes vor Auswertung des Materials vorgegeben werden, während weitere Kodes im Zuge der Auswertung erstellt werden können (Friese 2012).

In der Analyse wurden alle Textstellen erfasst, in denen Vokabular der drei semantischen Hauptfelder von Tourismus verwendet werden. Zur Erfassung des entsprechenden Vokabulars wurden zunächst zehn Texte Satz für Satz kodiert. Jede Textstelle, die einem der Kodes zugeordnet werden konnte, wurde erfasst; zudem wurden neue Kodes erstellt, um Textstellen thematisch zuordnen zu können.

Bei der Auswahl dieser zehn offen zu kodierenden Texte wurde darauf geachtet, dass möglichst aus jeder Fachrichtung, in deren Zusammenhang ein Tourismus-Ausdruck verwendet wurde, mindestens ein Artikel vertreten war. Ebenso wurde berücksichtigt, dass Autorinnen unterschiedlicher Fachbereiche vertreten waren, so dass fachlich bedingt unterschiedliches Vokabular erfasst werden konnte. Nach der Auswertung dieser zehn Texte wurde die Kodeliste strukturiert und entsprechend der in den Texten behandelten Themenbereiche erweitert.

Im nächsten Schritt wurde mittels des halbautomatischen Kodierens die gesamte hermeneutische Einheit (also alle 45 Artikel) nach Textstellen durchsucht, die eines der semantischen Felder von Tourismus bedienen (Friese 2012). Für das halbautomatische Kodieren wurden anhand der zuvor kodierten Texte Vokabellisten (sogenannte Fischernetze) zu den jeweiligen semantischen Feldern erstellt, nach denen alle Texte durchsucht wurden ${ }^{14}$. Jede gefundene Textstelle wurde überprüft und entsprechend kodiert oder verworfen. Textstellen konnten gleichzeitig mit mehreren Kodierungen versehen werden oder sich mit anderen Kodierungen überschneiden. Die Kodeliste konnte während der gesamten Auswertung verändert und ergänzt werden. So fiel zum Beispiel beim halbautomatischen Kodieren auf, dass das Fischernetz für das semantische Feld Ortswechsel viele Textstellen einfängt, in denen es um Behandlungen im Ausland geht, aber nicht explizit um die Reisetätigkeit einer Person. Diese Stellen wurden dem daraufhin neu erstellten Kode „Semantisches Feld: Ausland“ zugeordnet. Es wurden nur die Textstellen unter

\footnotetext{
${ }^{14}$ Beispielsweise export, supply, business für das semantische Feld Wirtschaft, exotic, leisure, vacation für das semantische Feld Erholung und travel, abroad, flight für das semantische Feld Ortswechsel.
} 
dem Kode „Semantisches Feld: Ortswechsel“ erfasst, die die aktive Handlung einer Reise einer oder mehrerer Personen beschreibt.

Die Textanalyse diente dazu, sowohl unterschiedliche Hyponyme, die in den Texten verwendet werden, als auch deren Definitionen zu erfassen. Ein weiterer Schwerpunkt lag auf der Sammlung derjenigen Textstellen, die eines der zuvor erarbeiteten semantischen Felder von Tourismus bedienen. Um die im Diskurs bestehenden Konnotationen zu dem Ausdruck Medizintourismus umfassend identifizieren zu können, wurden weitere Inhalte und formale Strukturen der Texte untersucht. Dies war durch die Methode des offenen Kodierens gewährleistet. Um alle verwendeten Hyponyme zu erfassen, wurde das Korpus mittels Auto-Coding ${ }^{15}$ nach dem Element „tour*“ durchsucht. Alle Treffer wurden einzeln überprüft und jedes unterschiedliche Hyponym einem jeweiligen Kode zugeordnet.

Mit Hilfe der systematischen Analyse des Textmaterials mit ATLAS.ti und in Anlehnung an die NCT-Methode von Friese lässt sich erfassen, ob und in welchem Ausmaß Vokabular, das den semantischen Feldern von Tourismus zugehörig ist, in Texten angewendet wird, die sich wissenschaftlich mit dem Phänomen reisender Patientinnen auseinandersetzen. In Kombination mit der Anwendung oben genannter Metapherntheorien soll durch die Analyse die These überprüft werden, dass die Metapher Medizintourismus unsere Perzeption des Phänomens reisender Patientinnen maßgeblich beeinflusst, und zwar vorrangig über implizite sprachliche Wirkung.

15 Mit der Funktion des Auto-Codings können alle zu analysierenden Dokumente automatisch auf bestimmte Wörter oder Wortteile hin durchsucht und diese dann entsprechenden Kodes zugeordnet werden. 


\subsection{MEDIZINTOURISMUS - REPRODUKTIONSTOURISMUS - TRANSPLANTATIONSTOURISMUS EINE LEITMETAPHER UND IHRE HYPONYME}

Die transnationale Medizin ist in ihrer heutigen Ausprägung ein neues Phänomen. Der Tourismus-Ausdruck drückt die Wahrnehmung der Produzentinnen in Bezug auf die touristischen Elemente im Gesamtkontext aus. Nach Kovács‘ Theorie der konstitutiven Leistung kommt es zur Bildung eines Metaphernsystems, das heißt, in sich kohärente Metaphern werden auf den neuen Sachverhalt übertragen und legen ihn damit auf die Strukturen des alten Zusammenhanges fest. Bei der Verwendung der Metapher Medizintourismus wird der Adressatin Tourismus als Deutungsrahmen vorgeschlagen. Tourismus stellt dabei nur die Leitmetapher dar. Indirekt werden weitere inhaltliche Übertragungen wie Patientin - Touristin, Krankenhaus - Hotel oder Reise Urlaub nahe gelegt. Dies zeigt sich sprachlich anhand von Ausdrücken wie "Medizintouristin“ oder „IVF-Holiday“ (Knoll 2008, S. 67). Die Theorie der Leitmetapher Tourismus mit nachfolgendem Metaphernsystem wurde durch die Auswertung des Textmaterials bestätigt: In den 45 analysierten Texten wurde zum Beispiel insgesamt 146-mal der Ausdruck Tourist/tourist für Patientinnen verwendet.

\subsubsection{AUSWERTUNG DER LITERATURRECHERCHE}

Um einen ersten Überblick über die Häufigkeiten des Ausdrucks Medizintourismus und der aus diesem Ausdruck abgeleiteten Hyponyme zu gewinnen, wurden die Titel der in der Literaturrecherche vom 10.02.2012 registrierten Dokumente ausgewertet. Die Recherche erfolgte in den Datenbanken BELIT und PubMed. Es wurden alle Texte erfasst, die das Stichwort tourism im Titel oder Abstract enthielten und zusätzlich mit mindestens einem MeSH-term, der zuvor als relevant ermittelt worden war, verschlagwortet waren. Von den so ermittelten 291 Texten verwendeten 234 Artikel einen Tourismus-Ausdruck im Titel. Diese Ausdrücke wurden erfasst und analysiert. Insgesamt wurden in den Titeln 26 verschiedene Ausdrücke verwendet. Tabelle 1 zeigt die Auflistung aller in den Titeln verwendeten Ausdrücke, in Synonymgruppen sortiert, und die jeweilige Anzahl der Dokumente, die den Ausdruck beinhalten. 
Tab. 1: Darstellung aller in der Recherche erfassten Hyponyme, in Synonymgruppen angeordnet. In der rechten Spalte findet sich die absolute Anzahl der Artikel, in denen der jeweilige Ausdruck verwendet wurde.

\begin{tabular}{|c|c|}
\hline $\begin{array}{l}\text { Ausdruck, der im Titel verwendet wird (Recher- } \\
\text { che vom } 10.02 .2012 \text { ) }\end{array}$ & Anzahl Dokumente \\
\hline Reproductive tourism, Reproduktionstourismus & 12 \\
\hline Procreative tourism & 4 \\
\hline Fertility tourism & 3 \\
\hline Assisted reproduction tourism & 1 \\
\hline Cosmetic surgery tourism & 1 \\
\hline Cosmetic tourism & 4 \\
\hline Sterbe-Tourismus & 1 \\
\hline Death tourism & 3 \\
\hline $\begin{array}{l}\text { Selbstmord-Tourist, Suicide tourism, Suicide } \\
\text { tourist }\end{array}$ & 5 \\
\hline Dental care tourism & 1 \\
\hline Dental implant tourism & 2 \\
\hline Dental tourism & 10 \\
\hline Health care tourism & 1 \\
\hline Health tourism, Health tourist & 7 \\
\hline $\begin{array}{l}\text { Medical tourism, Medical tourist, Medical tourist } \\
\text { patient, Medical tourism private hospital, Mediz- } \\
\text { intourismus, Medical tourism company, Medical } \\
\text { tourism facilitator }\end{array}$ & 100 \\
\hline Indian medical tourism & 1 \\
\hline Kidney transplant tourism & 1 \\
\hline Organ tourism & 2 \\
\hline Renal transplant tourism & 2 \\
\hline $\begin{array}{l}\text { Transplant tourism, Transplant tourist, Trans- } \\
\text { plantation tourism }\end{array}$ & 40 \\
\hline Cell therapy medical tourism & 1 \\
\hline Stem-cell tourism & 18 \\
\hline Science tourism, Science and production tourism & 2 \\
\hline Surgery tourism, Surgical tourism & 9 \\
\hline Vasectomy tourist & 1 \\
\hline Fistula tourism & 2 \\
\hline
\end{tabular}


Die Synonyme wurden anhand der Fachbereiche, auf die sich die Ausdrücke beziehen, ermittelt. In der folgenden Grafik sind die Summen der Dokumente, die einen Ausdruck aus der jeweiligen Synonymgruppe verwenden, dargestellt.

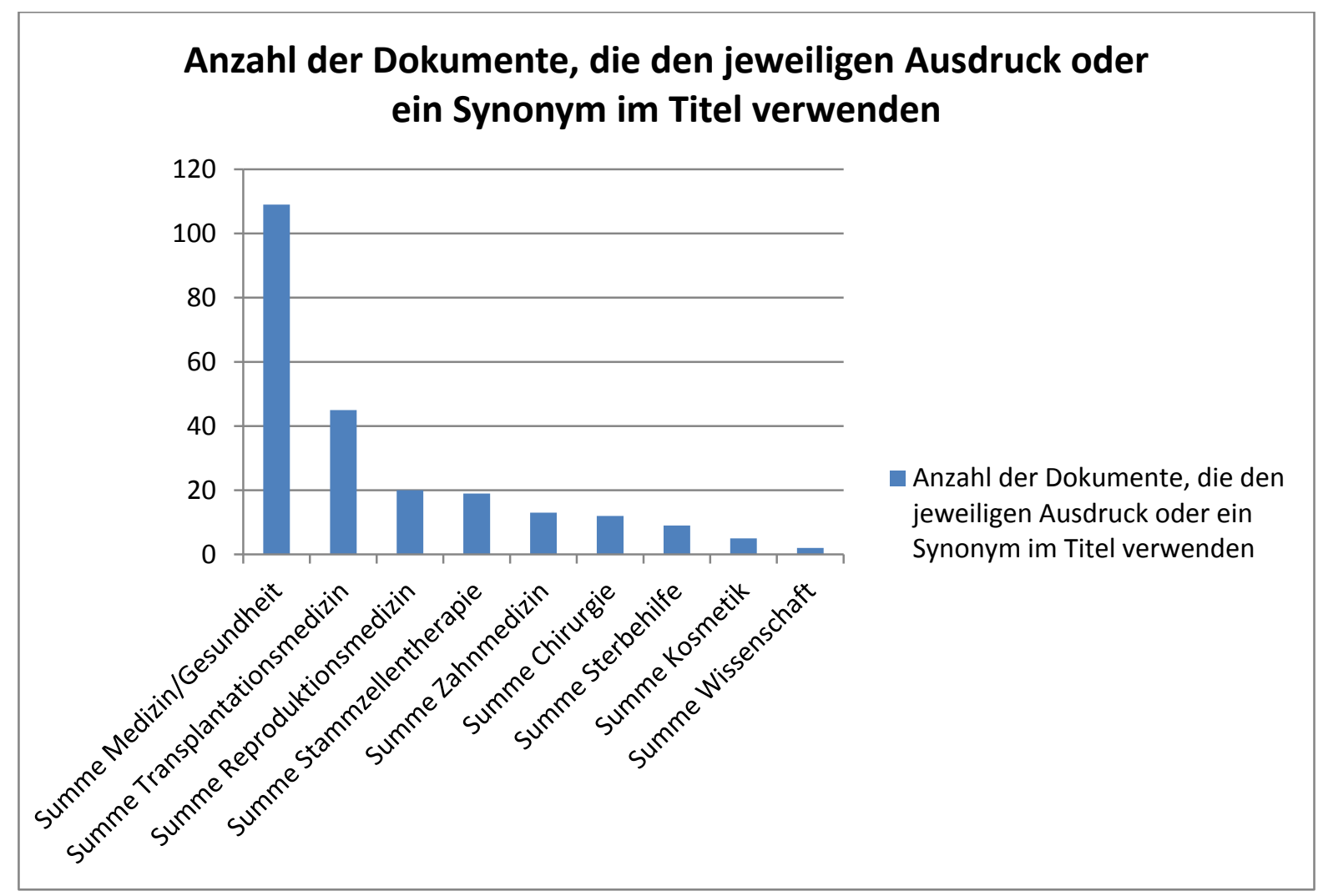

Abb. 2: Häufigkeit verschiedener Hyponyme im Vergleich

Der Ausdruck medical tourism (und die dazugehörigen Synonyme) ist mit einer Verwendung in 109 von 234 Titeln mit Abstand der häufigste Ausdruck, gefolgt von der Synonymgruppe transplant tourism mit 45 Titeln und reproductive tourism mit 20 Titeln. Die Erfassung der Ausdrücke in allen Titeln der Literaturrecherche soll vor allem dazu dienen, die Repräsentativität des Analysematerials zu überprüfen.

\subsubsection{AUSWERTUNG DES TEXTKORPUS}

Im Gegensatz zu der Erfassung der verwendeten Ausdrücke in den Titeln aller in der Literaturrecherche erfassten Dokumente sollten in einem Analysematerial von 45 Texten alle im Korpus (und nicht nur in den Titeln) verwendeten Ausdrücke erfasst werden. Mit der Funktion des Auto-Codings und dem Suchbegriff „tour*“ wurden alle im Korpus vorkommenden Hyponyme erfasst und einer Quantifizierung dahingehend, wie viele Texte welche Ausdrücke verwenden, ermöglicht. 
Es konnte festgestellt werden, dass insgesamt 16 unterschiedliche Hyponyme im Korpus verwendet werden, gebildet aus dem Wort Tourismus und einem Ausdruck aus dem medizinischen Wortfeld. Die Hyponymbildung folgt dabei den Regeln zur Bildung von Determinativkomposita, das heißt das erste Wort dient der Spezifizierung des zweiten. Dass die Hyponyme tatsächlich in Anlehnung an den Ausdruck medical tourism gebildet werden, lässt sich dadurch belegen, dass einige Autorinnen eine ausdrückliche Einordnung in das Phänomen medical tourism vornehmen. So schreibt zum Beispiel Eliza Barclay in einem Artikel über „stem-cell tourism“: „Stem-cell therapies are emerging as a growing area of medical tourism“ (Barclay 2009). Ebenso werden transplant tourism oder organ tourism zu medical tourism in Beziehung gesetzt: Der Transplantationsmediziner Roger W. Evans schreibt: „Transplant tourism, unlike other more benign forms of medical tourism, has become a flashpoint within the transplant community“ (Evans 2008, S. 1089). Auch Bennett Pafford und Shaun Pattinson verstehen medical tourism als Hyperonym für transplant/organ tourism: „While transplants are a small part of the overall medical tourism industry, the subject highlights some of the responsibilities individual countries have concerning international travel for medical treatment“ (Pafford 2009, S. 812); „'organ tourism“ is an aspect of organ trading, [...]. It is another instance of medical tourism, whereby patients, healthcare professionals and others travel in response to healthcare availability and demand in a globalised world“ (Pattinson 2008, S. 191). Srinivas beschreibt death tourism ebenfalls als „an instance of medical tourism“ (Srinivas 2009, S. 107-108).

Die Ergebnisse der Hyponym-Erfassung werden in Tabelle 2 mit der Gesamtanzahl ihrer Verwendung und der Anzahl der Artikel, die den jeweiligen Ausdruck verwenden, dargestellt. 
Tab. 2: Häufigkeit, mit der die einzeln aufgeführten Ausdrücke in den Texten benutzt werden. Die dritte Spalte zeigt die Anzahl der Artikel, in denen der jeweilige Ausdruck verwendet wird.

\begin{tabular}{|l|r|r|}
\hline Ausdruck & Anzahl insgesamt & $\begin{array}{l}\text { Anzahl Dokumente, } \\
\text { die Ausdruck ver- } \\
\text { wenden }\end{array}$ \\
\hline Abortion tourism & 2 & 1 \\
\hline Arthroplasty tourism & 1 & 1 \\
\hline Cosmetic tourism & 8 & 2 \\
\hline Death tourism & 57 & 1 \\
\hline Dental tourism & 24 & 2 \\
\hline Fertility tourism & 10 & 2 \\
\hline Health tourism & 2 & 1 \\
\hline Health care tourism & 2 & 2 \\
\hline Medical tourism & 265 & 31 \\
\hline Organ tourism & 3 & 1 \\
\hline Procreative tourism & 3 & 3 \\
\hline Reproductive tourism & 20 & 3 \\
\hline Stem-cell tourism & 13 & 2 \\
\hline Suicide tourism & 1 & 1 \\
\hline Surgical tourism & 25 & 4 \\
\hline Transplant tourism / Transplanta- & 173 & 17 \\
tionstourismus & & \\
\hline
\end{tabular}

In einem zweiten Schritt wurde nach inhaltlichen Überschneidungen der Hyponyme und nach Synonymen innerhalb der Hyponyme gesucht. Die Ergebnisse sind in Tabelle 3 dargestellt. 
Tab. 3: Häufigkeit, mit der die Ausdrücke in den Texten benutzt wurden. In dieser Darstellung sind die Ausdrücke unterschiedlichen medizinischen Fachbereichen zugeordnet und in Synonymgruppen sortiert aufgeführt. Die dritte Spalte zeigt die Anzahl der Artikel, in denen ein Ausdruck aus der entsprechenden Synonymgruppe verwendet wurde.

\begin{tabular}{|c|c|c|c|}
\hline Bereich & Synonyme Ausdrücke & $\begin{array}{l}\text { Anzahl, wie oft } \\
\text { ein Ausdruck im } \\
\text { Korpus verwendet } \\
\text { wurde: Synonyme } \\
\text { gesamt }\end{array}$ & $\begin{array}{l}\text { Anzahl Dokumen- } \\
\text { te gesamt }\end{array}$ \\
\hline Chirurgie & Surgical tourism & 25 & 4 \\
\hline Orthopädie & Arthroplasty tourism & 1 & 1 \\
\hline Plastische Chirurgie & Cosmetic tourism & 8 & 2 \\
\hline Sterbehilfe & $\begin{array}{l}\text { Death tourism, Suicide tou- } \\
\text { rism }\end{array}$ & 58 & 2 \\
\hline Zahnmedizin & Dental tourism & 24 & 2 \\
\hline Reproduktionsmedizin & $\begin{array}{l}\text { Fertility tourism, Procreative } \\
\text { tourism, Reproductive tour- } \\
\text { ism }\end{array}$ & 33 & 4 \\
\hline Abtreibung & Abortion tourism & 2 & 1 \\
\hline $\begin{array}{l}\text { Gesundheitsversorgung } \\
\text { allgemein }\end{array}$ & $\begin{array}{l}\text { Medical tourism, Health tour- } \\
\text { ism, Health care tourism }\end{array}$ & 269 & 32 \\
\hline Transplantation & $\begin{array}{l}\text { Organ tourism, Transplant } \\
\text { tourism / Transplantations- } \\
\text { tourismus }\end{array}$ & 176 & 18 \\
\hline Stammzelltherapie & Stem-cell tourism & 13 & 2 \\
\hline
\end{tabular}

Die fett gedruckten Wörter stellen die innerhalb einer Synonymgruppe am häufigsten verwendeten Ausdrücke dar. Im weiteren Textverlauf steht die Nennung eines der fettgedruckten Wörter immer für die gesamte Synonymgruppe.

Zusammenfassend lässt sich festhalten, dass eine Vielzahl an Hyponymen im Diskurs verwendet wird. In dem Korpus von 45 Texten wurden 16 unterschiedliche Ausdrücke gefunden, wovon medical tourism (269), transplant tourism (176) und death tourism (58) in absoluten Zahlen am häufigsten verwendet wurden. Sieht man auf die Anzahl der Dokumente, ergibt sich eine andere Verteilung: Medical tourism wird in 32 von 45 Dokumenten verwendet, transplant tourism in 18 von 45 Dokumenten und reproductive tourism und surgical tourism jeweils in 4 von 45 Dokumenten. Diese unterschiedliche Verteilung ist auf den unterschiedlichen Umfang der Artikel zurückzuführen. Um festzustellen, welche Ausdrücke im Diskurs verbreitet sind, ist der Blick auf die Dokumentenanzahl hilfreicher, da es sonst zu Fehlinterpretationen kommen kann. So stellt death tourism mit 58 Treffern eine hohe Zahl dar, was die Annahme nahelegte, es handele sich um einen verbreiteten Ausdruck, tatsächlich wird er nur in 2 von 45 Texten erwähnt. 
Die Häufigkeitsverteilung der Hyponyme in den Dokumenten entspricht in den ersten drei Positionen der in der Literaturrecherche ermittelten. In Bezug auf die Häufigkeiten der Hyponyme ist das Analysematerial also repräsentativ für die in dem Zeitraum 2000 bis 2012 in den Datenbanken PubMed und BELIT erfassten Artikel.

\subsection{MEDIZINTOURISMUS: EIN AUSDRUCK - VIELFÄLTIGE BEDEUTUNGEN}

\subsubsection{EXPLIKATIONEN ${ }^{16}:$ FORMALE ASPEKTE}

Um die Bedeutungen und Konnotationen des Ausdrucks Medizintourismus und der dazugehörigen Hyponyme zu erarbeiten, lag ein Schwerpunkt bei der Auswertung des Textmaterials auf den Explikationen der Autorinnen. Aus dem gesamten Korpus wurden alle Textstellen, in denen die Bedeutung von Medizintourismus und/oder eines Hyponyms erläutert wird, herausgearbeitet und analysiert.

Insgesamt konnten in 22 von 45 Dokumenten 27 Explikationen ermittelt werden. In jeweils 9 Dokumenten werden die Ausdrücke medical tourism und transplant tourism definiert, in 4 Texten wird reproductive tourism definiert, die Ausdrücke surgical tourism, death tourism, cosmetic tourism, stem-cell tourism und dental tourism werden jeweils einmal definiert. $\mathrm{Zu}$ fast jedem Hyponym liegt also mindestens eine Explikation vor, nur zu den Ausdrücken arthroplasty tourism und abortion tourism gibt es im Korpus keine Explikation.

Von den 27 Explikationen nehmen 10 Bezug auf im Diskurs bereits bestehende Explikationen oder Definitionen. Auffällig ist, dass dies vor allem in den Explikationen der Ausdrücke transplant tourism und reproductive tourism der Fall ist. 5 von 9 Explikationen von transplant tourism beziehen sich auf andere Explikationen. 2 davon rekurrieren auf eine Explikation von Shimazono. 3 von 4 Explikationen von reproductive tourism beziehen sich auf andere Explikationen, zwei davon auf eine von Pennings. Darüber hinaus bezieht sich die einzige Explikation von death tourism und 1 von 9 Explikationen von medical tourism auf andere Explikationen.

Bevor auf die inhaltlichen Aspekte der Explikationen eingegangen wird, wird dargelegt, welche Autorinnen präzisieren. Es könnte zum Beispiel sein, dass das Fachgebiet der Autorinnen Einfluss darauf hat, ob die Bedeutung des Ausdrucks herausgearbeitet wird. Um diese Hypothese zu

\footnotetext{
${ }^{16}$ Ich gehe in dieser Arbeit davon aus, dass es sich bei Medizintourismus um einen Ausdruck aus der vorwissenschaftlichen Alltagssprache handelt, der noch nicht hinreichend definiert ist. In den Texten liegen dennoch Präzisierungen des Ausdrucks vor, nur wenige Autorinnen benennen diese Präzisierungen als Definition. Deshalb verwende ich für die im Korpus vorgenommenen Präzisierungen von Medizintourismus den Ausdruck Explikation. Der Ausdruck Definition wird nur dann verwendet, wenn die entsprechende Passage im Originaltext ausdrücklich als solche gekennzeichnet ist, die Autorinnen also selbst den Anspruch auf eine Definition erheben.
} 
überprüfen, war die Einteilung aller Dokumente in Dokumentenfamilien nach fachlichem Hintergrund der Autorinnen hilfreich. Mit Hilfe der Software ATLAS.ti konnte die Abfrage gemacht werden, welchen fachlichen Hintergrund die Autorinnen der Texte mit Explikationen haben ${ }^{17}$. Die Ergebnisse sind in Tabelle 4 dargestellt:

Tab. 4: Zusammenhang von fachlichem Hintergrund der Autorin und Explikation des benutzten Tourismus-Ausdrucks

\begin{tabular}{|l|l|l|l|}
\hline $\begin{array}{l}\text { Dokumentenfamilie: fach- } \\
\text { licher Hintergrund der } \\
\text { Autorinnen }\end{array}$ & $\begin{array}{l}\text { Anzahl Do- } \\
\text { kumente aus } \\
\text { dem Korpus } \\
(\mathrm{n}=45)\end{array}$ & $\begin{array}{l}\text { Anzahl der Do- } \\
\text { kumente mit } \\
\text { Explikation } \\
(\mathrm{n}=22)\end{array}$ & $\begin{array}{l}\text { Anteil der Dokumente } \\
\text { der jeweiligen Fachrich- } \\
\text { tung mit Explikation (in } \\
\text { Prozent) }\end{array}$ \\
\hline Anthropologie & 3 & 3 & 100 \\
\hline Recht & 3 & 2 & 67 \\
\hline Medizin/Zahnmedizin & 27 & 15 & 56 \\
\hline Ethik/Philosophie & 6 & 3 & 50 \\
\hline Journalismus & 4 & 1 & 25 \\
\hline Wirtschaft/Management & 5 & 1 & 20 \\
\hline
\end{tabular}

Es lässt sich festhalten, dass in allen Texten von Autorinnen mit anthropologischem Hintergrund Explikationen vorliegen. 67\% der Texte von Autorinnen mit rechtswissenschaftlichem Hintergrund enthalten eine Explikation. In 56\% der Texte von Autorinnen mit medizinischem Hintergrund liegen Explikationen vor, gefolgt von 50\% der Texte von Autorinnen mit ethischem/philosophischem Hintergrund. In 1 von 4 Texten von Autorinnen aus dem JournalismusBereich wird eine Explikation vorgenommen, bei den Texten der Dokumentenfamilie Hintergrund Wirtschaft/Management ist dies nur bei 1 von 5 Texten der Fall.

\subsubsection{EXPLIKATIONEN: INHALTLICHE ASPEKTE}

Um die inhaltlichen Aussagen der Explikationen vergleichen zu können, wurden aus allen Explikationen die einzelnen, das Phänomen charakterisierenden Aussagen herausgearbeitet und auf Überschneidungen zu anderen Explikationen geprüft. Dabei ergab sich eine Menge von 36 unterschiedlichen charakterisierenden Aussagen zu Medizintourismus und/oder den Hyponymen.

Tabelle 5 zeigt, inwiefern sich die Explikationen der unterschiedlichen Ausdrücke in ihren Aussagen überschneiden, unterscheiden oder sogar widersprechen. Es ist dafür nicht notwendig,

\footnotetext{
17 Dazu wurde jede Dokumentenfamilie als Auswertungsrahmen gesetzt (die Dokumentenfamilie erfüllt damit eine Filterfunktion). Dann wurde nach allen Dokumenten (in der entsprechenden Dokumentenfamilie) gesucht, die einen Kode „Explikation“ enthalten. So konnte jeweils die Anzahl von Artikeln einer Dokumentenfamilie (zum Beispiel fachlicher Hintergrund: Medizin) mit einer Explikation von Medizintourismus und/oder eines Hyponyms ermittelt werden.
} 
alle Aussagen anzuführen, der Übersichtlichkeit halber werden in Tabelle 5 exemplarisch fünf Aussagen und ihre Häufigkeitsverteilung in den Explikationen ausführlich dargestellt.

Tab. 5: Häufigkeiten bestimmter Aussagen in den Explikationen.

\begin{tabular}{|l|r|r|}
\hline Charakterisierende Aussagen & \multicolumn{2}{|c|}{$\begin{array}{l}\text { Anzahl der Explikationen, in denen die entspre- } \\
\text { chende Aussage vorgenommen wird (n=27) }\end{array}$} \\
\hline $\begin{array}{l}|c| \\
\text { Personen erhalten außerhalb ihres Wohnortes } \\
\text { medizinische Behandlungen. }\end{array}$ & 27 & 100 \\
\hline Es handelt sich um internationale Bewegungen. & 10 & 30 \\
\hline $\begin{array}{l}\text { Personen umgehen das geltende Recht des } \\
\text { Wohnortes. }\end{array}$ & 4 & 37 \\
\hline $\begin{array}{l}\text { Personen führen außerhalb ihres Wohnortes } \\
\text { medizinische Behandlungen durch beziehungs- } \\
\text { weise wechseln ihren Wohnort, um an einem } \\
\text { anderen Ort/in einem anderen Land zu praktizie- } \\
\text { ren. }\end{array}$ & 4 & 15 \\
\hline Es handelt sich um ein Gewerbe. & 3 & 11 \\
\hline
\end{tabular}

Es wird deutlich, dass sich die Explikationen in ihren Aussagen unterscheiden. Zum Beispiel betrachten 13 Artikel internationale Bewegungen als charakterisierend für das Phänomen, nur 3 Explikationen stimmen in der Aussage, es handele sich bei dem Phänomen um ein Gewerbe, überein. Während 4 Artikel auch Fachkräfte, die im medizinischen Bereich tätig sind und ihren Wohnort wechseln, um zu arbeiten, in ihre Explikation einschließen, beziehen sich die übrigen Explikationen ausschließlich auf Patientinnen. Eine Aussage findet sich jedoch in allen Explikationen: „Personen erhalten außerhalb ihres Wohnortes medizinische Behandlungen“. Als kleinster gemeinsamer Nenner aller Explikationen lässt sich festhalten, dass es immer mindestens um einen Ortswechsel aus medizinischen Gründen geht. Diese inhaltliche Analogie aller Explikationen entspricht dem semantischen Hauptaspekt Reise des Ausdrucks Tourismus.

Unklar ist dabei jedoch, auf wen sich der Reiseaspekt beziehen soll. Dass diese Frage unterschiedlich beantwortet werden kann, zeigt sich in der untersuchten Fachliteratur anhand der unterschiedlichen Explikationen. Kantor zum Beispiel bezieht sich in ihrem Text auf Patientinnen und Patienten, die ihren Heimatort für eine medizinische Behandlung verlassen (Kantor 2008). In dem Aufsatz „Beyond Medical Tourism: Authentic Engagement in Global Health“ gehen Anderson und Wansom hingegen ausschließlich auf Medizinstudierende ein, die einen Teil ihres Studiums im Ausland verbringen (Anderson und Wansom 2009). 
Shaun Pattinson bezieht Patientinnen, Expertinnen und pauschal weitere Personen, die im Zusammenhang mit medizinischer Versorgung reisen, in ihre Explikation von Transplantationstourismus mit ein:

\begin{abstract}
'Organ tourism' is an aspect of organ trading, if it is understood as involving travel to other jurisdictions for the purpose buying or selling organs [sic]. It is another instance of medical tourism whereby patients, healthcare professionals, and others travel in response to healthcare availability and demand in a globalised world (Pattinson 2008, S. 191) (Hervorhebung durch M.M.).
\end{abstract}

Mit dem Tourismus-Ausdruck werden jedoch nicht nur reisende Spenderinnen, Empfängerinnen oder Transplantationsexpertinnen erfasst. Einige Autorinnen schließen in ihre Explikationen nicht nur Personen, sondern auch über Grenzen hinweg transportierte Organe, Ei- oder Samenzellen mit ein (The Transplantation Society und International Society of Nephrology 2008; Inhorn und Patrizio 2009). So wird zum Beispiel in der Declaration of Istanbul eine weite Definition vorgestellt, die nicht nur unterschiedliche Personengruppen, sondern auch den Transfer von Organen umfasst: „Travel for transplantation is the movement of organs, donors, recipients or transplant professionals across jurisdictional borders for transplantation purposes" (The Transplantation Society und International Society of Nephrology 2008, S. 2) (Hervorhebung durch M.M.).

Die Aussagen, die in den Explikationen getroffen werden, sind vielfältig und zum Teil unvereinbar unterschiedlich. Während zum Beispiel in drei Explikationen von Transplantationstourismus das Phänomen als illegal charakterisiert wird, räumt Budiani-Saberi ein, es gebe auch Transplantationstourismus, der „legal and appropriate“ sei (Budiani-Saberi und Delmonico 2008, S. 926). Eine weitere Uneinigkeit in den Explikationen zeigt sich daran, welche den Ort wechselnde Personen beziehungsweise Dinge in die Explikation mit eingeschlossen werden. Während in einigen Explikationen ausschließlich Patientinnen, die für medizinische Behandlungen ihren Wohnort verlassen, eingeschlossen werden, nehmen andere Autorinnen weitere Explikationen vor. Diese reichen von der Berücksichtigung von den Wohnort verlassendem Fachpersonal bis hin zu Organen, Eizellen, oder Samenzellen, die über Grenzen transportiert werden. Einige Explikationen schließen auch third parties (also nicht nur Ei- oder Samenzellen, sondern auch Leihmütter, Samenspender und Eizellspenderinnen) und Organspenderinnen, die ihren Wohnort für die Behandlung anderer Personen verlassen, mit ein. So wird transplant tourism in der Declaration of Istanbul folgendermaßen definiert:

Travel for transplantation is the movement of organs, donors, recipients or transplant professionals across jurisdictional borders for transplantation purposes. Travel for transplantation becomes transplant tourism if it involves organ trafficking and/or transplant commercialism or if the resources (organs, professionals and transplant centers) devoted to providing transplants to patients from outside a country undermine the country's ability to provide transplant services for its own population (The Transplantation Society und International Society of Nephrology 2008, S. 2). 
Inhorn und Patrizio ziehen in ihrem Text eine Explikation des Juristen Storrow heran: „Fertility tourism may also occur in the reverse, when the infertile import the third parties necessary for their fertility treatment“" (Inhorn und Patrizio 2009, S. 904).

Wie im vorangegangenen Abschnitt erwähnt, werden in einigen Explikationen Transplantationstourismus, legaler Transplantationstourismus und travel for transplantation unterschieden. Die Aussagen zu den Explikationen dieser Ausdrücke wurden ebenfalls erfasst. Die Explikationen von legalem Transplantationstourismus (P7) und travel for transplantation (P9) sind nahezu identisch und werden insofern von Transplantationstourismus unterschieden, als es um Lebendspenden zwischen Verwandten geht. Diese leben entweder in unterschiedlichen Ländern oder haben in ihrem Heimatland keinen Zugang zur Transplantationsmedizin, weshalb die Operation im Ausland erfolgen müsse. Morphologisch ist travel for transplantation deutlicher von dem Ausdruck Transplantationstourismus abgegrenzt, da es sich nicht um ein Determinativkompositum handelt. Auch die Chirurgin Claragh Healy, die in ihrem Text den Ausdruck surgical tourism verwendet, unterscheidet zwischen „practice of travelling“ und „burgeoning industry“ und bekräftigt, der Ausdruck „surgical tourism“ beziehe sich auf beides (Healy 2009, S. 125). Sie unterscheidet also zwei verschiedene Phänomene, die mit demselben Ausdruck beschrieben werden. Healy beschreibt unterschiedliche Bedeutungen des Ausdrucks ohne die Vieldeutigkeit zu problematisieren. Der Ausdruck wird von ihr weder explizit kritisiert, noch macht sie den Vorschlag, weitere oder andere Ausdrücke einzuführen.

Zusammenfassend kann gezeigt werden, dass lediglich in der Hälfte der Artikel (48,89\%) eine oder mehrere Explikationen des Ausdrucks Medizintourismus beziehungsweise eines der Hyponyme vorgenommen wurde, obwohl alle Texte den Ausdruck im Titel oder Abstract verwenden. Bei näherer Betrachtung der Explikationen konnte gezeigt werden, dass diese in ihren Aussagen deutlich voneinander abweichen. Als einzige übereinstimmende Aussage aller Explikationen zeigte sich der Aspekt des Ortswechsels von Patientinnen für medizinische Behandlungen. Dabei ist dieser Aspekt als kleinster gemeinsamer Nenner zu werten, da einige Autorinnen deutlich weitere Explikationen vornehmen. Im nächsten Abschnitt soll untersucht werden, ob die Vielzahl unterschiedlicher Explikationen in den unterschiedlichen Bedeutungen und Assoziationen einzelner Hyponyme begründet ist.

\subsubsection{EXPLIKATIONEN: INHALTLICHE ASPEKTE IN ABHÄNGIGKEIT VON DEN HYPONYMEN}

Um zu untersuchen, inwiefern die Vielzahl unterschiedlicher Aussagen abhängig von Bedeutungsunterschieden der Hyponyme ist, wurden die Explikationen der drei am häufigsten erläuterten Ausdrücke, medical tourism, transplant tourism und reproductive tourism, miteinander verglichen. 
Tabelle 6 zeigt dieselben Aussagen wie Tabelle 5, es werden aber nur noch die Explikationen der drei am häufigsten genannten Ausdrücke berücksichtigt und nebeneinander aufgeführt. Es zeigen sich zum Teil deutliche Übereinstimmungen in den Aussagen zu den Explikationen abhängig von einzelnen Hyponymen.

Tab. 6: Häufigkeit bestimmter Aussagen in den Explikationen in Abhängigkeit des charakterisierten Hyponyms.

\begin{tabular}{|l|c|c|c|}
\hline Charakterisierende Aussagen & \multicolumn{3}{|c|}{$\begin{array}{c}\text { Anzahl der Explikationen des jeweiligen Ausdrucks, in denen } \\
\text { die entsprechende Aussage vorgenommen wird }\end{array}$} \\
\hline & $\begin{array}{c}\text { medical tourism } \\
(n=9)\end{array}$ & $\begin{array}{c}\text { transplant tourism } \\
(n=9)\end{array}$ & $\begin{array}{c}\text { reproductive tou- } \\
\text { rism (n=4) }\end{array}$ \\
\hline $\begin{array}{l}\text { Personen erhalten außerhalb ihres } \\
\text { Wohnortes medizinische Behand- } \\
\text { lungen. }\end{array}$ & $9(100 \%)$ & $9(100 \%)$ & $4(100 \%)$ \\
\hline $\begin{array}{l}\text { Es handelt sich um internationale } \\
\text { Bewegungen. }\end{array}$ & $6(67 \%)$ & $9(100 \%)$ & $0(0 \%)$ \\
\hline $\begin{array}{l}\text { Personen umgehen das geltende } \\
\text { Recht des Wohnortes. }\end{array}$ & $0(0 \%)$ & $7(78 \%)$ & $2(50 \%)$ \\
\hline $\begin{array}{l}\text { Personen führen außerhalb ihres } \\
\text { Wohnortes medizinische Behand- } \\
\text { lungen durch bzw. wechseln ihren } \\
\text { Wohnort, um an einem anderen } \\
\text { Ort/in einem anderen Land zu prak- } \\
\text { tizieren. }\end{array}$ & $1(11 \%)$ & $3(33 \%)$ & $0(0 \%)$ \\
\hline Es handelt sich um ein Gewerbe. & $2(22 \%)$ & $0(0 \%)$ & $0(0 \%)$ \\
\hline
\end{tabular}

Wie auch in Tabelle 5 wird als einzige Analogie aller Explikationen die Aussage „Personen erhalten außerhalb ihres Wohnortes medizinische Behandlungen“ deutlich.

Die Aussage, dass es sich um internationale Reisen handele, findet sich in allen Explikationen von transplant tourism, während dieser Punkt in den Explikationen von reproductive tourism kein einziges Mal erwähnt wird. Darüber hinaus ist transplant tourism in 78\% der Fälle über die Aussage charakterisiert, es handele sich um Reisen von dem Wirkungsbereich einer Gesetzgebung in den Wirkungsbereich einer anderen. Dieser Aspekt spielt hingegen in den Explikationen von medical tourism keine Rolle, wird aber auch in 50\% der Explikationen von reproductive tourism erwähnt. Die Aussage, es handele sich bei dem Phänomen um ein Gewerbe, wird ausschließlich in den Explikationen von medical tourism gemacht.

Es ist anzunehmen, dass die Vielfältigkeit der Explikationen nicht nur auf die unterschiedlichen Schwerpunkte in den jeweiligen Kontexten der unterschiedlichen Hyponyme, sondern auf die Interdisziplinarität der ethischen Diskussion zurückzuführen ist. Zum Beispiel liegt die Hypothese nahe, dass Autorinnen mit rechtswissenschaftlichem Hintergrund eher die Aussage „Die 
Gesetzgebung am Wohnort unterscheidet sich von der am Zielort" als charakterisierend betrachten als Autorinnen anderer Fachrichtungen. Zwar wurde im Zuge der Auswertung auch der fachliche Hintergrund der Autorinnen erfasst, die Anzahl der Texte ist jedoch zu gering, als dass eine repräsentative Auswertung in diesem Punkt möglich wäre.

Wenn auch die Anzahl der Artikel mit Explikationen der jeweiligen Hyponyme klein ist, lässt sich ein Trend zu unterschiedlichen charakterisierenden Aussagen je nach Hyponym feststellen, obwohl alle auf die Leitmetapher Medizintourismus zurückgehen.

Nachdem in diesem Kapitel der Bedeutungsumfang von Medizintourismus und einiger zugehöriger Hyponyme anhand von Explikationen ermittelt und diskutiert wurde, wird im Folgenden untersucht, ob die Semantik von Tourismus in Texten, in denen die Metapher Medizintourismus verwendet wird, von Bedeutung ist.

\subsection{SEMANTISCHE ASPEKTE VON TOURISMUS IM KONTEXT VON MEDIZINTOURISMUS}

Um den Einfluss der Semantik von Tourismus im Zusammenhang mit der Verwendung der Metapher Medizintourismus herauszuarbeiten, wird das Textkorpus daraufhin untersucht, ob die zuvor erarbeiteten semantischen Hauptaspekte von Tourismus (Orstwechsel, Wirtschaftszweig, Freizeit) in den Texten eine Rolle spielen. Zum einen wird das Vokabular des gesamten Textmaterials auf die drei semantischen Hauptaspekte untersucht, zum anderen werden die Explikationen im Hinblick auf das darin verwendete Vokabular gesondert betrachtet. In der Diskussion werden diese Ergebnisse mit Hilfe der Metapherntheorie von Kovács interpretiert. Dabei wird herausgearbeitet, ob der Ausdruck Medizintourismus die verschiedenen Funktionen einer Metapher erfüllt.

Zunächst wurde das Analysematerial systematisch auf Vokabular aus den semantischen Feldern Wirtschaft, Ortswechsel und Freizeit ${ }^{18}$ untersucht und entsprechende Textstellen kodiert. Für die Auswertung der Semantik der Explikationen wurden alle Textstellen, die sowohl mit einem der drei Kodes, die die drei Hauptaspekte von Tourismus darstellen, als auch mit dem Kode Explikation versehen sind, näher betrachtet. Da alle Explikationen in der Aussage übereinstimmen, es handele sich um Patientinnen, die außerhalb ihres Wohnortes medizinische Behandlungen erhalten, überrascht es nicht, dass alle Explikationen sich auf Vokabular aus mindestens einem der drei berücksichtigten semantischen Felder von Tourismus beziehen (semantisches Feld Reise/Ortswechsel). Es bleibt also die Frage, inwieweit Vokabular aus den semantischen Feldern Wirtschaft und Freizeit/Erholung in den Explikationen verwendet wird. Die Antwort lautet,

\footnotetext{
${ }^{18}$ Beispielsweise export, supply, business für das semantische Feld Wirtschaft, exotic, leisure, vacation für das semantische Feld Erholung und travel, abroad, flight für das semantische Feld Ortswechsel.
} 
dass in 12 der 27 Explikationen das semantische Feld Wirtschaft bedient wird und in nur 2 Explikationen Vokabular aus dem semantischen Feld Freizeit/Erholung zur Anwendung kommt. Zur Verdeutlichung ist dieses Ergebnis in Abbildung 3 grafisch dargestellt.

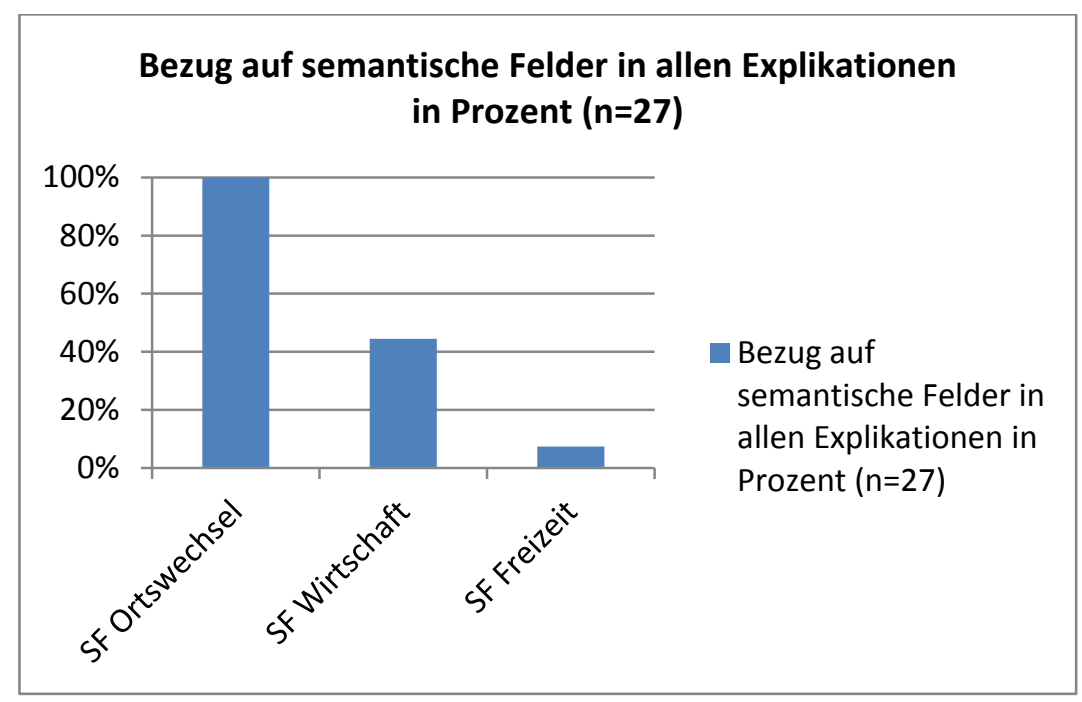

Abb. 3: Häufigkeit des Vokabulars des jeweiligen semantischen Feldes in den Explikationen.

Des Weiteren interessiert, ob bei den Explikationen der Hyponyme Unterschiede in der Verwendung von Vokabular der entsprechenden semantischen Felder auftreten. Dazu wurden die Explikationen der am häufigsten genannten Ausdrücke medical tourism, transplant tourism und reproductive tourism in der Verwendung der semantischen Felder zugehörigen Vokabulars miteinander verglichen.

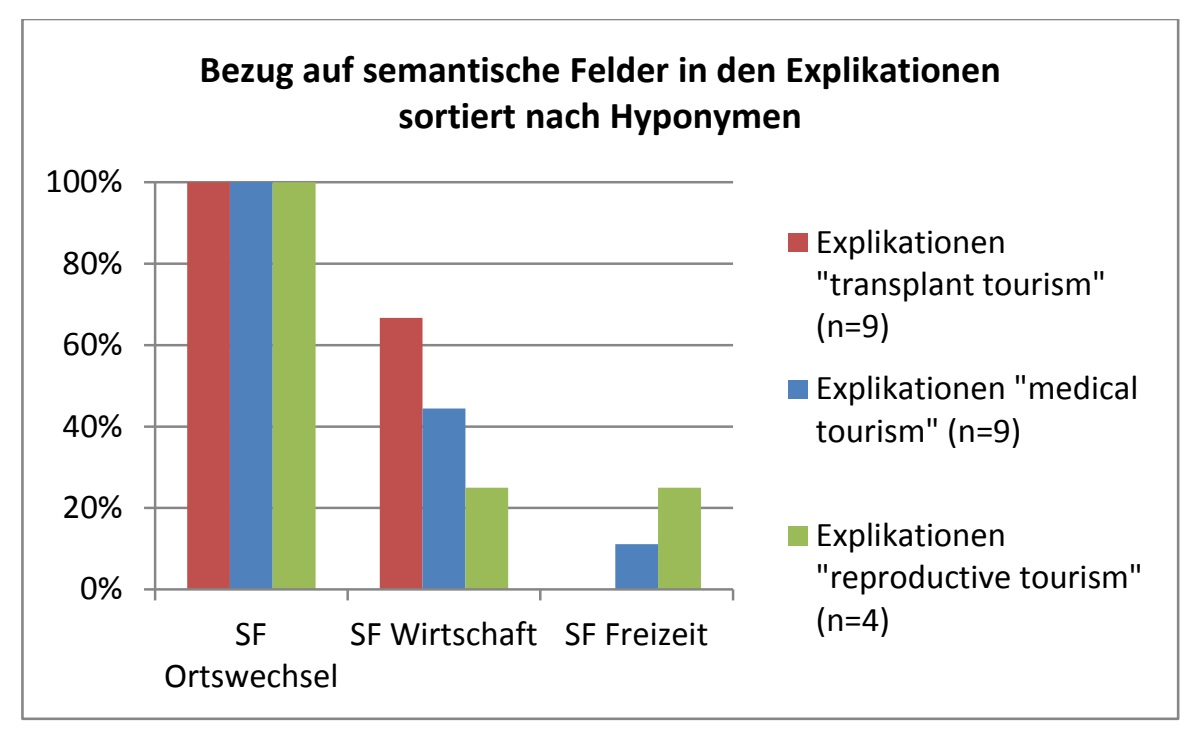

Abb. 4: Häufigkeit der semantischen Felder in den Explikationen der Hyponyme transplant tourism, medical tourism und reproductive tourism 
Abbildung 4 veranschaulicht, dass eine unterschiedliche Gewichtung der semantischen Felder in den Explikationen abhängig von den Hyponymen erfolgt. Unterschiede zeigen sich vor allem im semantischen Feld Wirtschaft. So wird Vokabular aus diesem semantischen Feld in 6 von 9 Explikationen von transplant tourism herangezogen, während es in nur 1 von 4 Explikationen von reproductive tourism verwendet wird. Vokabular aus dem semantischen Feld Freizeit findet insgesamt weniger Berücksichtigung, in den Explikationen von transplant tourism wird es gar nicht eingesetzt, in den Explikationen von medical tourism und reproductive tourism jeweils einmal. Allerdings muss berücksichtigt werden, dass in dieser Explikation von reproductive tourism das semantische Feld Freizeit zwar bedient wird, jedoch explizit als Kritik an dem Ausdruck und mit Bezug auf eine Explikation von Storrow, dass „pleasure travel“ in der Regel nicht der Realität der Betroffenen entspreche (Inhorn und Patrizio 2009). So bleibt unter den Explikationen nur eine von medical tourism, die Vokabular aus dem semantischen Feld Freizeit tatsächlich in charakterisierender Absicht verwendet: „Today's popular use of the term 'medical tourism' describes two types of activities: one where consumers take exotic vacations and combine them with surgery, and another where consumers choose to go abroad to find more affordable health care services“ (Kantor 2008, S. 1-2) (Hervorhebung durch M.M.).

Zusammenfassend lässt sich feststellen, dass in den Explikationen Vokabular der semantischen Felder der drei Hauptaspekte von Tourismus zu finden ist, wobei das semantische Feld Ortswechsel überwiegt. Inhaltlich stellt also der Reiseaspekt die einzige Analogie aller Explikationen dar.

Die semantischen Felder Wirtschaft und Freizeit werden in den Explikationen seltener bedient. Bei dem semantischen Feld Wirtschaft fällt eine unterschiedlich häufige Anwendung auf, abhängig davon, welches Hyponym charakterisiert wird. Da es sich bei den Explikationen der Hyponyme um eine geringe Anzahl handelt, können lediglich Tendenzen festgestellt werden. Während das semantische Feld Wirtschaft in 67\% der Explikationen von transplant tourism bedient wird, findet es in nur 25\% der Explikationen von reproductive tourism Berücksichtigung. 44\% Prozent der Explikationen von medical tourism benutzen Vokabular aus dem semantischen Feld Wirtschaft, obwohl nur 22\% Explikationen medical tourism ausdrücklich als Gewerbe charakterisieren. In den Explikationen von transplant tourism ist diese Diskrepanz zwischen ausdrücklichen inhaltlichen Aussagen und Verwendung des Vokabulars noch deutlicher: Während keine der 9 Explikationen von transplant tourism eine Charakterisierung als Gewerbe vornimmt, verwenden 6 von 9 Explikationen Vokabular aus dem semantischen Feld Wirtschaft.

Im Folgenden wird die Verwendung von Vokabular der semantischen Felder im gesamten Textmaterial aufgezeigt. Alle Texte wurden auf die drei semantischen Aspekte Freizeit, Ortswechsel 
und Wirtschaft untersucht. Insgesamt wurden für das semantische Feld Freizeit 63 Textstellen kodiert, für das semantische Feld Ortswechsel 392 und für das semantische Feld Wirtschaft 584 Stellen.

Es wird deutlich, dass in Texten, in denen der Ausdruck Medizintourismus (oder ein Hyponym) verwendet wird, auf die drei semantischen Hauptaspekte des Ausdrucks Tourismus Bezug genommen wird. Um zu untersuchen, ob die unterschiedlichen semantischen Felder unterschiedlich gewichtet werden, wurden zum einen, wie oben erwähnt, die absolute Anzahl der Zitate mit den jeweiligen Kodierungen erfasst, zum anderen wurde die Anzahl der Texte ermittelt, die auf das jeweilige semantische Feld Bezug nehmen. Dabei ergab sich, dass 43 von 45 Texten sich auf das semantische Feld Wirtschaft beziehen, 42 Texte den Reise-Aspekt erwähnen, während nur 20 Texte Vokabular aus dem semantischen Feld Freizeit einsetzen.

Die Ergebnisse werden in der Abbildung 5 veranschaulicht.

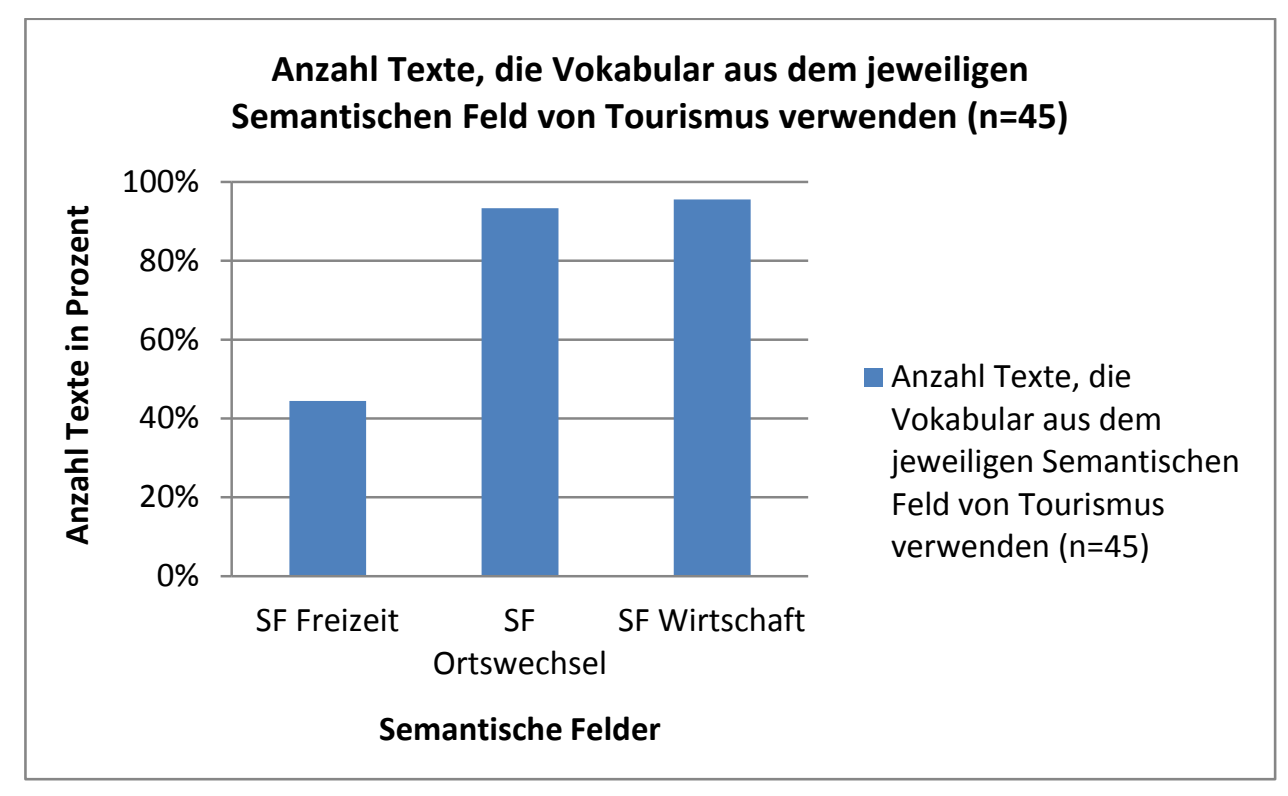

Abb. 5: Anzahl der Texte, die Vokabular der jeweiligen semantischen Felder aufweisen.

Vergleicht man Abbildung 5 mit Abbildung mit Abbildung 3, die die Verwendung von Vokabular aus den semantischen Feldern in den Explikationen darstellt, fällt auf, dass das semantische Feld Wirtschaft im gesamten Korpus anteilig häufiger verwendet wird als in den Explikationen. Zur Veranschaulichung dieser Abweichung werden die Ergebnisse der Abbildungen 5 und 3 in der folgenden Grafik (Abbildung 6) gegenübergestellt: 


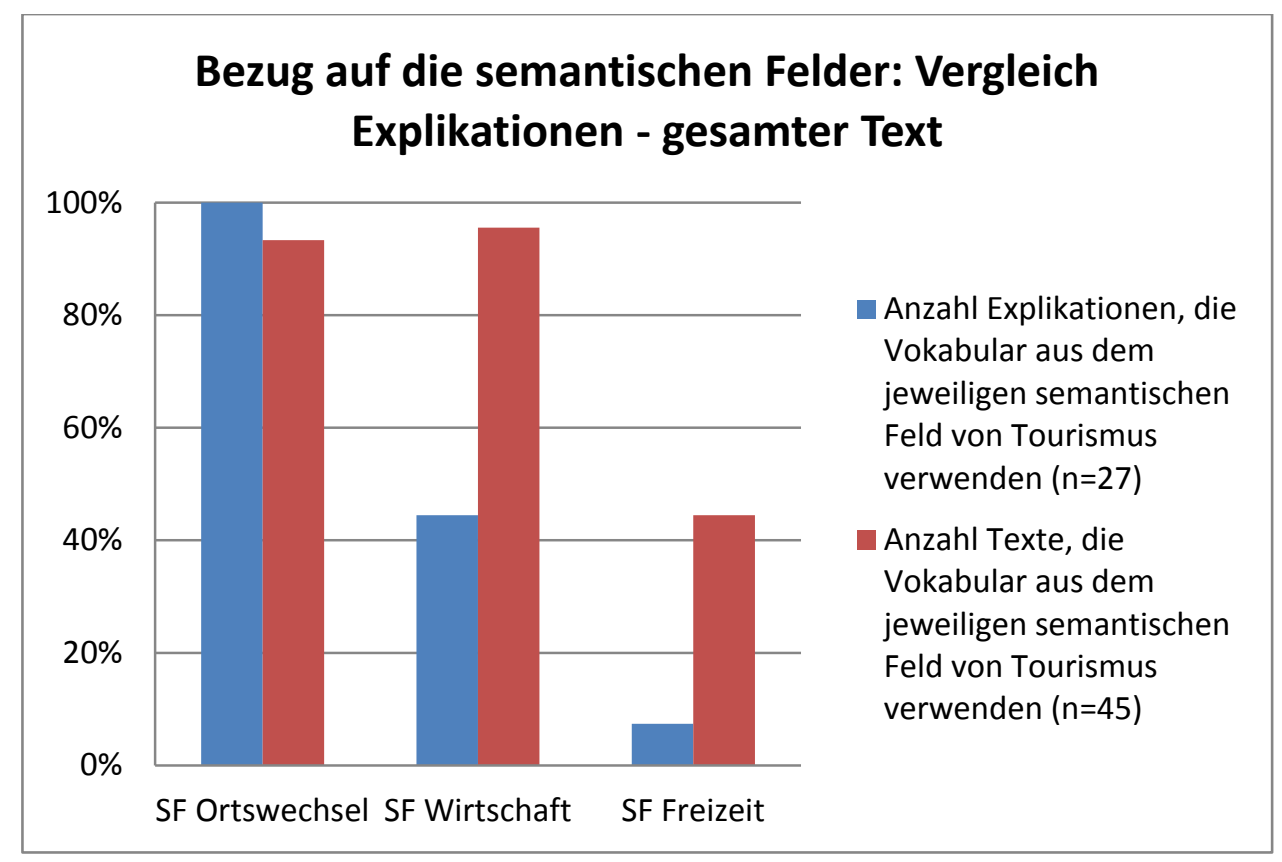

Abb. 6: Gegenüberstellung der Anwendung der semantischen Felder in den Explikationen und im gesamten Textkorpus.

Verglichen mit dem Wirtschafts-Aspekt, auf den 95,56\% der Texte Bezug nehmen, wird der Freizeit-Aspekt mit 44,44\% deutlich seltener erwähnt. Beide Aspekte werden im gesamten Korpus jedoch häufiger berücksichtigt als in den Explikationen. Das heißt, obwohl diese zwei semantischen Hauptaspekte in den Explikationen von Medizintourismus deutlich seltener als die Ortswechselanalogie erwähnt werden, prägt das Vokabular der semantischen Felder Wirtschaft und Freizeit die fachliche Diskussion über das mit der Metapher Tourismus beschriebene Phänomen. Diese Verteilung lässt sich nicht nur anhand der Anzahl Texte, die auf die semantischen Felder zurückgreifen, zeigen, sondern stimmt mit dem Verhältnis der absoluten Anzahl der dem jeweiligen semantischen Feld zugeordneten Zitate überein. Dieses wird mit Hilfe der Abbildung 7 illustriert. 


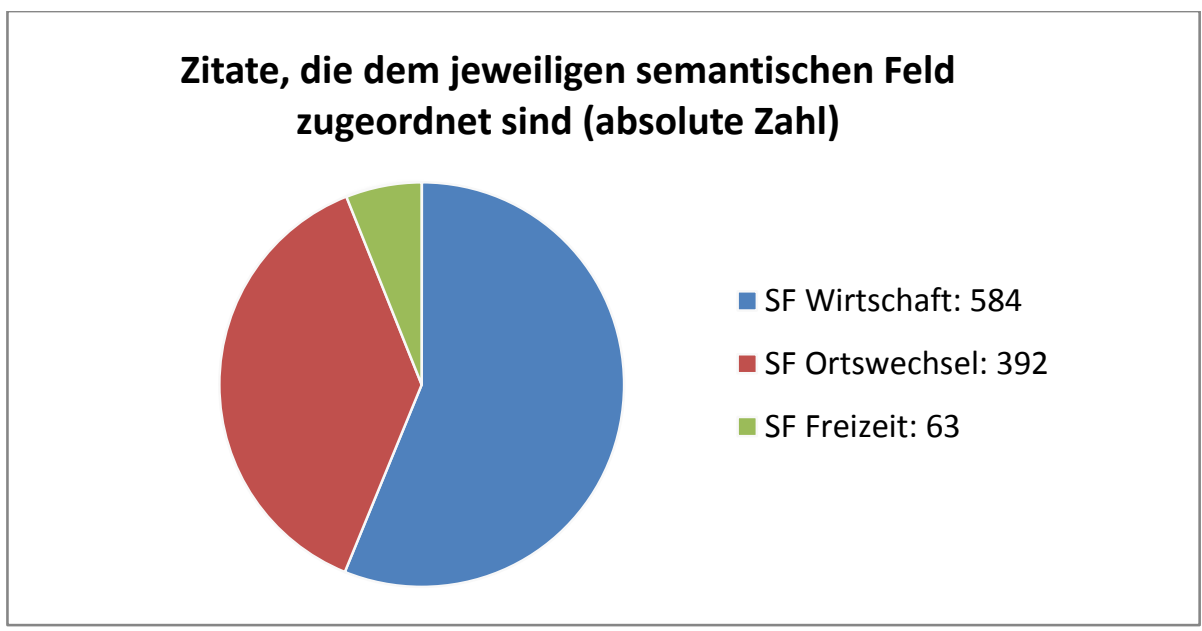

Abb. 7: Anteil der den jeweiligen semantischen Felder zugeordneten Zitate im gesamten Textkorpus (absolute Anzahl).

Zusammenfassend kann festgestellt werden, dass in den Explikationen das semantische Feld Ortswechsel und damit die Analogie zum Reiseaspekt gegenüber dem Wirtschafts- und Freizeitaspekt überwiegt. Im gesamten Korpus überwiegt jedoch deutlich der Wirtschaftsaspekt gegenüber den semantischen Feldern Ortswechsel und Freizeit.

\subsection{GEGENPROBE: DER LEIDASPEKT}

Nach Kovács‘ Theorie zu den Metaphernfunktionen zeigt sich die konstitutive Funktion einer Metapher daran, dass die Konzeption des neuen Sachverhaltes auf den Deutungsrahmen der Metapher festgelegt wird. Dies führt im Kontext der vorliegenden Arbeit zu der These, dass Aspekte, die nicht in der Bedeutung des Ausdrucks Tourismus verankert sind, weniger Berücksichtigung in der ethischen Diskussion des Themas finden. Dies gilt vor allem für diejenigen Texte, die den Ausdruck Medizintourismus oder ein Hyponym verwenden. Gleichzeitig bedeutet dies, dass Texte, die sich mit der Thematik von reisenden Patientinnen auseinandersetzen ohne die Metapher zu verwenden, vermutlich Aspekte außerhalb der Semantik von Tourismus stärker berücksichtigen. Das Leid der Betroffenen stellt im Zusammenhang mit zu medizinischen Zwecken Reisenden einen solchen Aspekt dar. In einer Untersuchung von Texten aus den öffentlichen Medien konnten Sharon Bassan und ich diese These bereits bestätigen. So konnten wir zeigen, dass Texte, die die Perspektive der Reisenden stärker berücksichtigen, den Ausdruck Tourismus eher vermeiden (Bassan und Michaelsen 2013).

Da in der vorliegenden Arbeit nur Artikel erfasst wurden, die den Ausdruck Medizintourismus oder eines seiner Hyponyme verwenden, können unterschiedliche Themenschwerpunkte zwischen Texten, die den Ausdruck verwenden und solchen, die die Metapher vermeiden, nicht untersucht werden. Dennoch kann in dieser Arbeit die konstitutive Funktion anhand des semanti- 
schen Feldes Leid untersucht werden. Denn die Patientinnenrolle schließt zunächst Leiderfahrungen mit ein. Diese können durch konkrete Beeinträchtigungen durch eine bestimmte Krankheit bedingt sein, zum Beispiel Gelenkschmerzen bei Arthrose oder jahrelange Dialyse bei Niereninsuffizienz. Auch ein unerfüllter Kinderwunsch kann Ursache von Leid sein. Dazu kommt selbstverständlich die unmittelbare Leiderfahrung während des Auslandsaufenthalts, die durch die Behandlung hervorgerufen werden kann, etwa Schmerzen oder Komplikationen nach einer Operation. Dass Patientinnen und Patienten, die zu medizinischen Zwecken reisen, den Ausdruck Tourismus für ihre Situation als nicht angemessen empfinden, zeigten Inhorn und Patrizio in einer qualitativ-empirischen Studie mit Patienten und Patientinnen, die aus reproduktionsmedizinischen Gründen ins Ausland gereist sind. Diese empfanden ihre Reise als „highly stressfull“ und sind der Meinung, der Ausdruck Tourismus verhöhne ihr Leiden (Inhorn und Patrizio 2009, S. 905). Durch den Ausdruck werden die reisenden Personen als Touristinnen konzeptionalisert, doch diese Sichtweise entspricht nicht der Wirklichkeit der Betroffenen. Sie machen wie alle Patientinnen Leiderfahrungen, die durch die Verwendung dieses Ausdrucks in den Hintergrund rücken, da Leid nicht Teil der Semantik von Tourismus ist.

Leid stellt also einen Aspekt dar, der in dem Ausdruck Tourismus keine Berücksichtigung findet, wohl aber in den Feldern der Medizin und der Gesundheitsversorgung. Dies führt zu der These, dass die konstitutive Funktion der Metapher Medizintourismus dazu führt, dass das semantische Feld Leid in den Texten seltener bedient wird als die semantischen Felder Ortswechsel, Wirtschaftszweig und Freizeit. Um die konstitutive Funktion der Metapher zu überprüfen, wurden also alle 45 Texte nach dem semantischen Feld Leid untersucht.

Die Überprüfung des semantischen Feldes Leid ergab, dass 30 von 45 Texten Vokabular aus diesem semantischen Feld verwenden, im Gegensatz zu 21 Texten, die das semantische Feld Freizeit bedienen. Dieses Ergebnis scheint die These der konstitutiven Funktion zu widerlegen: Das semantische Feld Leid gehört im Gegensatz zu dem semantischen Feld Freizeit nicht zu Tourismus und wird in der ethischen Debatte dennoch häufiger verwendet. Bei näherer Betrachtung der Ergebnisse der Verwendung von Vokabular aus dem semantischen Feld Leid fällt jedoch auf, dass das Leiden unterschiedlicher Betroffener berücksichtigt wird. So berichten Texte, die den Ausdruck transplant tourism verwenden, zum einen über das Leid der Organempfängerinnen, zum anderen über das Leid der Organspenderinnen. Je nach verwendetem Hyponym werden also unterschiedliche Leidaspekte berücksichtigt: Texte, die andere Hyponyme als transplant tourism verwenden, erwähnen den Leidaspekt im Zusammenhang mit dem Leid der reisenden Patientinnen. 
Aufgrund dieser inhaltlich unterschiedlichen Schwerpunkte werden die Ergebnisse des semantischen Feldes Leid in folgende drei Gruppen sortiert: Leid allgemein, Leid Empfängerinnen und Leid Spenderinnen. Dabei wurde das Leid der reisenden Patientinnen aus allen anderen Bereichen außer der Transplantationsmedizin in der Gruppe Leid allgemein zusammengefasst.

Die Ergebnisse der Verwendung von Vokabular aus dem semantischen Feld Leid werden in Tabelle 7 dargestellt.

Tab. 7: Häufigkeit der Texte mit Vokabular aus den jeweiligen semantischen Feldern

\begin{tabular}{|l|l|}
\hline Semantisches Feld Leid & Anzahl der Dokumente $(n=30)$ \\
\hline Leid allgemein & $15(33 \%)$ \\
\hline Leid Empfängerin & $11(24 \%)$ \\
\hline Leid Spenderin & $16(36 \%)$ \\
\hline
\end{tabular}

Insgesamt nehmen 30 Texte Bezug auf einen der drei Leidaspekte, dabei beziehen sich 15 Texte auf das Leiden von Patientinnen allgemein, 11 auf Empfängerinnen und 16 auf das Leiden der Spenderinnen.

Auch in diesem Fall wird nicht nur die Anzahl der Texte, sondern die absolute Anzahl der gefundenen Zitate berücksichtigt, da sie ein Indiz dafür sind, in welchem Maße die unterschiedlichen Aspekte berücksichtigt werden. Für Leid allgemein wurden insgesamt 50 Zitate gefunden, das semantische Feld Leid im Zusammenhang mit Empfängerinnen wurde 33-mal bedient und im Zusammenhang mit Spenderinnen 67-mal. In Abbildung 8 werden alle bisher untersuchten semantischen Felder vergleichend dargestellt. 


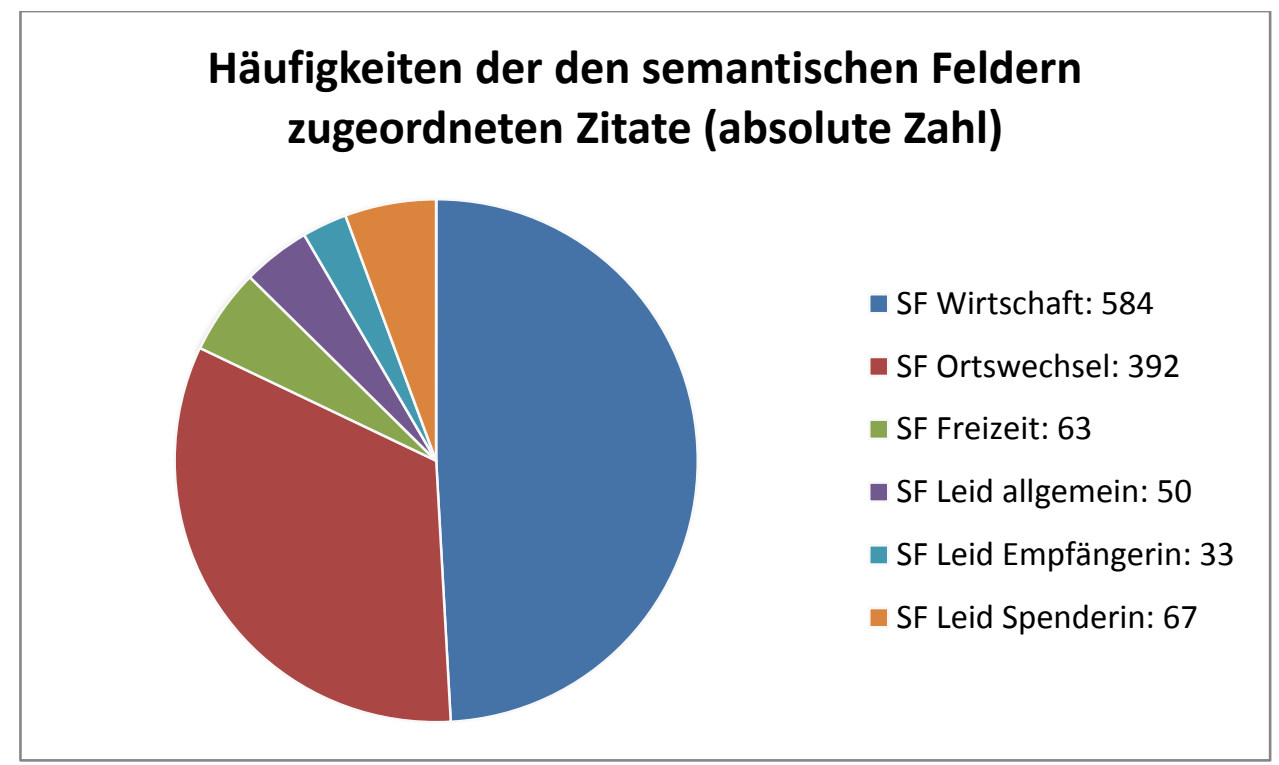

Abb. 8: Anteil der den jeweiligen semantischen Felder zugeordneten Zitate im gesamten Textkorpus (absolute Anzahl)

Die Grafik zeigt, dass das semantische Feld Wirtschaft am häufigsten bedient wird, gefolgt vom semantischen Feld Ortswechsel. Das semantische Feld Leid Spenderinnen liegt noch vor dem semantischen Feld Freizeit, dann folgen das semantische Feld Leid allgemein und zuletzt Leid Empfängerinnen. Im Vergleich zu den häufig bedienten Feldern Wirtschaft und Ortswechsel wird Leid Empfängerinnen also außerordentlich selten erwähnt. 


\section{Bedeutungen und Nebenbedeutungen DeR Metapher}

\section{MEDIZINTOURISMUS}

Gerade in einem interdisziplinären Feld wie der Ethik ist es wichtig, dass Einigkeit über die Bedeutung von Schlüsselwörtern in der Diskussion herrscht. Bei neuen Ausdrücken wie Medizintourismus ist es deshalb unerlässlich, dass die jeweiligen Autorinnen erläutern, wie sie einen bestimmten Ausdruck verstehen. Die Textanalyse hat jedoch gezeigt, dass in der ethischen Diskussion weniger als die Hälfte der Autorinnen, die den Ausdruck Medizintourismus oder einen daraus abgeleiteten Ausdruck (Hyponym) verwenden, den jeweils verwendeten Ausdruck präzisieren. Die genauere Untersuchung der wenigen Explikationen ergab eine Vielfalt an Bedeutungen und Nebenbedeutungen des Ausdrucks Medizintourismus. Dass ein so großer Bedeutungsumfang problematisch ist, zeigt sich besonders deutlich anhand des Ausdrucks transplant tourism: Während einige Autorinnen darunter nur illegalen Organhandel verstehen, beziehen andere legale Organtransplantationen über Ländergrenzen hinweg mit ein. Auch die Vielzahl an Hyponymen von Abtreibungstourismus bis Sterbetourismus trägt zu einer großen Bandbreite und Ungenauigkeit des Ausdrucks bei. Deshalb werden im Folgenden die Hyponyme unter Bezug auf ihre Leitmetapher Medizintourismus diskutiert. Im Anschluss daran wird die Bedeutung der Semantik von Tourismus in der Anwendung von Medizintourismus herausgearbeitet. Dazu werden Anwendungsbeispiele der semantischen Felder Ortswechsel, Wirtschaft und Freizeit im Zusammenhang mit dem Ausdruck Medizintourismus aufgezeigt und diskutiert.

Des Weiteren werden die Nebenbedeutungen (Konnotationen) mit Hilfe von Kovács Metapherntheorie herausgearbeitet. Ein Schwerpunkt der Diskussion liegt auf der Darstellung der unterschiedlichen Verwendungsweisen der Metapher, da sich hieran die Normativität des Ausdrucks Medizintourismus besonders gut zeigen lässt. Im Anschluss daran wird gezeigt, inwiefern der Gebrauch dieser Metapher unser Verständnis von Medizin in einer globalisierten Welt prägt. Auf der Suche nach einem alternativen Ausdruck werden schließlich Bedeutungen und Nebenbedeutungen anderer Ausdrücke gegeneinander abgewogen.

\subsection{DiE EXPLIKATIONEN}

In nur 22 von 45 analysierten Texten finden sich Explikationen des Ausdrucks Medizintourismus beziehungsweise seiner Hyponyme. Für das Verständnis eines jeden Diskurses ist es aber von großer Wichtigkeit, dass die verwendeten Ausdrücke definiert werden. Die Ethik zeichnet sich 
durch Interdisziplinarität aus; die vielen beteiligten Fächer tragen zwar jeweils wichtige Aspekte zur Diskussion bei, allerdings muss man gerade deshalb davon ausgehen, dass Vorwissen und Vorstellungen von Teilnehmenden zu einer bestimmten Thematik stark variieren. Mit Blick auf die Interdisziplinarität der ethischen Diskussion überrascht es, dass nur wenige Autorinnen den Ausdruck Medizintourismus kritisch diskutieren und präzisieren, zeigen doch die unterschiedlichen Explikationen, dass die Vorstellungen, was unter diesem Ausdruck zu verstehen sei, deutlich voneinander abweichen. Gerade in wissenschaftlichen Texten sollte das Vokabular sorgfältig gewählt und auf Nebenbedeutungen überprüft werden.

Im Folgenden wird anhand konkreter Explikationen von transplant tourism aus dem analysierten Textkorpus die Varianz der charakterisierenden Aussagen aufgezeigt. Daran anschließend wird dargelegt, inwiefern die Vielzahl unterschiedlicher Hyponyme problematisch ist.

\subsubsection{MEHRDEUTIGKEIT - ILLUSTRIERT AM BEISPIEL VON TRANSPLANT TOURISM}

Die Bandbreite dessen, was die Autorinnen für die Explikation des Phänomens als wichtig erachten, zeigt sich sowohl an der hohen Zahl unterschiedlicher Aussagen als auch an der geringen Zahl von Überschneidungen. Wie ich weiter oben dargestellt habe, werden von den insgesamt 36 charakterisierenden Aussagen zu Medizintourismus beziehungsweise seiner Hyponyme 13 nur in jeweils einer Explikation gemacht. Durch die Analyse der Explikationen wurde außerdem deutlich, dass es keine als allgemein gültig anerkannte Explikation gibt. Nur 10 der 27 Explikationen berücksichtigen überhaupt Explikationen anderer Autorinnen. Die größten Unterschiede weisen die Explikationen zu transplant tourism auf und werden deshalb im Folgenden ausführlich besprochen.

Für den Ausdruck transplant tourism liegen im untersuchten Korpus 9 Explikationen vor. In diesen 9 Explikationen kann man 21 unterschiedliche inhaltliche Aussagen differenzieren. 10 davon werden in nur jeweils einer Explikation erwähnt. Es liegt also eine große Varianz darüber vor, was die Autorinnen als wichtig für die Charakterisierung des mit transplant tourism bezeichneten Phänomens erachten. Eine der bekanntesten Explikationen von transplant tourism stellt diejenige aus der Declaration of Istanbul dar. Dennoch rekurrieren nur 8 von 18 Texten, die den Ausdruck transplant tourism verwenden, auf diese Definition. Gleichzeitig gibt es für kein anderes Hyponym eine so hohe Zahl an unterschiedlichen charakterisierenden Aussagen. Selbst wenn also eine Definition bekannt ist, wie in diesem Fall diejenige aus der Declaration of Istanbul, bedeutet dies keine Einigkeit über die inhaltlichen Aussagen, was unter dem Ausdruck transplant tourism zu verstehen sei. Darüber hinaus sind die einzelnen Explikationen keineswegs eindeutig: Während Budiani-Saberi und Delmonico zum Beispiel transplant tourism zu- 
nächst als eine Form von Organhandel definieren, den sie zuvor wiederum als illegal definiert haben, erwähnen sie danach ausdrücklich, dass es auch transplant tourism gebe, der „legal and appropriate" sei:

\begin{abstract}
Transplant Tourism has become a connotation for organ trafficking. The United Network for Organ Sharing (UNOS), recently defined transplant tourism as 'the purchase of a transplant organ abroad that includes access to an organ while bypassing laws, rules, or processes of any or all countries involved' (3). However, not all medical tourism that entails the travel of transplant recipients or donors across national borders is organ trafficking. Transplant tourism may be legal and appropriate. Examples include, when travel of a related donor and recipient pair is from countries without transplant services to countries where organ transplantation is performed or if an individual travels across borders to donate or receive a transplant via a relative. Any official regulated bilateral or multilateral organ sharing program is not considered transplant tourism if it is based on a reciprocated organ sharing program among jurisdictions. (Budiani-Saberi und Delmonico 2008, S. 926)
\end{abstract}

Diese Definition ist verwirrend, denn Legalität wird zwar in der Definition mehrfach als Kriterium genannt, kann als Definitionskriterium jedoch nicht standhalten, da transplant tourism sowohl illegal als auch legal sein kann. Die eigentlichen Definitionsmerkmale wie „purchase of a transplant organ abroad" rücken in den Hintergrund. Der Adressatin werden die wirklich definierenden Aussagen nicht mehr deutlich.

Nicht nur Budiani-Saberi und Delmonico, sondern insgesamt sieben von neun Autorinnen, die transplant tourism präzisieren, bringen transplant tourism mit illegalem Organhandel in Verbindung. Im Gegensatz dazu verstehen wir unter Tourismus eigentlich einen legalen Wirtschaftszweig. Hier versuchen also die Autorinnen, die ursprüngliche Bedeutung der Metapher als legalen Wirtschaftszweig zu illegalem Handel umzudeuten. Auch die Transplantation Society erklärt in ihrer Definition, transplant tourism beinhalte „organ trafficking and/or transplant commercialism“, wobei organ trafficking illegalen Organhandel meint (The Transplantation Society und International Society of Nephrology 2008, S. 2). Es wäre für das Verständnis der jeweiligen Texte dringend notwendig, dass Autorinnen die genutzten Ausdrücke präzisieren. In diesem Fall übersehen die Autorinnen jedoch, dass die Konnotationen einer Metapher über Gemeinplätze eines Ausdrucks wahrgenommen werden. Diese Gemeinplätze sind im Sprachgebrauch verankert und können nicht willkürlich umgedeutet werden. Sie bleiben zusätzlich zu einer Explikation bestehen und beeinflussen das Verständnis des neuen Sachverhaltes. In diesem Falle bedeutet dies, dass transplant tourism von der Adressatin zunächst als legales Wirtschaftssystem wahrgenommen wird und illegale Aspekte übersehen werden.

Die inhaltlichen Aussagen in der Definition der Transplantation Society von transplant tourism können nicht im Sinne der semantischen Hauptaspekte Tourismus als Freizeitgestaltung beziehungsweise Tourismus als legaler Wirtschaftszweig interpretiert werden. Es liegt ein metaphorischer Gebrauch des Ausdrucks Tourismus vor, der sich auf wenige Analogien zwischen ur- 
sprünglichem und neuem Sachverhalt bezieht. Die metaphorische Verwendung von transplant tourism bezieht sich zum einen auf die Reiseaktivität der Patientinnen, zum anderen auf kommerzielle Aspekte. Diese kommerziellen Aspekte sind im Zusammenhang von Patientinnen, die zu Transplantationszwecken reisen, zumeist illegal, dennoch können sie mit Vokabular beschrieben werden, das zu der ursprünglichen Semantik von Tourismus als Wirtschaftszweig gehört. So wird schließlich aus einer Organspenderin eine Verkäuferin und aus einer Empfängerin eine Transplantationstouristin:

Moreover, global transplant commercialism has failed to deliver on its promises to the 'tourists' (transplant recipients) themselves, with outcome studies indicating increased morbidity mainly due to poor vendor screening, selection, and matching in the organ-exporting country complicated even further by poor record keeping (Epstein 2009, S. 135) (Hervorhebungen durch M.M.).

Da im internationalen Organhandel tatsächlich kommerzielle Strukturen vorhanden sind, wirkt der Gebrauch von Vokabular aus dem wirtschaftlichen Bereich schlüssig. Dass es sich dabei um illegale kommerzielle Strukturen handelt, wird im Sprachgebrauch häufig nicht deutlich. Die Illegalität des Organhandels ist in der Semantik der Wortbausteine Transplantation und Tourismus nicht angelegt. Weder Transplantationen an sich noch Tourismus sind illegal. Durch die Verwendung von Vokabular aus dem semantischen Feld Wirtschaft wird suggeriert, es handele sich um legale kommerzielle Strukturen: „Transplants conducted in countries with loose or no legal frameworks such as that of Pakistan, the Philippines and Egypt accommodate the organ market and the transplant tourists that drive the demand" (Budiani-Saberi und Delmonico 2008, S. 928) (Hervorhebung durch M.M.).

So schreiben Budiani-Saberi und Delmonico zwar von rechtlich wenig oder nicht regulierten Räumen, in vielen Ländern gilt bei Organhandel jedoch das Extraterritorialitätsprinzip ${ }^{19}$, das heißt, den einreisenden Patientinnen ist es nicht erlaubt, ein Organ zu kaufen, unabhängig von der Rechtslage des Ziellandes. Selbst wenn es sich also um rechtliche Grauzonen in den Zielländern handelt, handeln die reisenden Patientinnen häufig illegal. Dieser illegale Handel wird mit dem Ausdruck Tourismus verschleiert. Zwar versuchen einige Autorinnen in ihren Explikationen transplant tourism als illegalen Organhandel zu definieren, sie übersehen dabei jedoch, dass die Adressatin zum Verständnis einer Metapher immer auch die ursprünglichen Gemeinplätze heranziehen wird, in diesem Falle die Bedeutung Tourismus = legaler Wirtschaftszweig. Die Botschaften einer Metapher werden über Gemeinplätze vermittelt. Aufgrund dieser impliziten

\footnotetext{
${ }^{19}$ Extraterritorialität beschreibt einen Rechtsstatus, nach dem eine Person nicht derjenigen Hoheitsgewalt unterliegt, auf deren Territorium sie sich befindet. Dieses Prinzip ist nur bei einigen wenigen Gesetzen wirksam, da es immer einen Eingriff in die Souveränität anderer Staaten bedeutet. Am Beispiel des Verbotes von Organhandel bedeutet dies, dass deutsche Bürgerinnen, wenn sie an nach deutschem Gesetz illegalem Organhandel beteiligt sind, überall auf der Welt rechtlich so behandelt werden, als würden sie sich auf deutschem Gebiet aufhalten.
} 
Wirkweise kann es nicht gelingen, eine Metapher entgegen ihrer ursprünglichen Semantik, in diesem Fall Tourismus als legaler Wirtschaftszweig zu Tourismus als illegalem Organhandel, umzudefinieren.

Abgesehen von den inhaltlich voneinander abweichenden Explikationen von transplant tourism zeigt sich die Uneindeutigkeit des Ausdrucks daran, dass einige Autorinnen versuchen, die unterschiedlichen Kontexte durch unterschiedliche Ausdrücke voneinander zu unterscheiden. Dabei fällt auf, dass diese Ausdrücke nun nicht mehr als Hyponyme des Ausdrucks medical tourism imponieren, sie aber dennoch den semantischen Hauptaspekten Ortswechsel und Wirtschaft zugeordnet werden können.

Solche Ausdrücke werden in den Explikationen von transplant tourism meist eingeführt, um legale Transplantationen in Verbindung mit Ortswechsel von Beteiligten von illegalen Transplantationen abzugrenzen. Transplant tourism wird dabei häufig als illegal charakterisiert. Dadurch entsteht im Wortschatz eine Lücke für die legalen Transplantationen mit reisenden Patientinnen. Dies ist zum Beispiel der Fall, wenn eine Verwandte in einem anderen Land lebt, zur Lebendspende aber in das Heimatland der Empfängerin reist, um dort innerhalb eines Transplantationsprogrammes eine Niere zu spenden. In der Declaration of Istanbul wird zum Beispiel der Ausdruck travel for transplantation als übergeordneter Ausdruck eingeführt. Travel for transplantation umfasst sowohl legale als auch illegale Transplantationen in Verbindung mit reisenden Patientinnen. Transplant tourism setzt hingegen „organ trafficking and/or transplant commercialism“ voraus. Organ trafficking wird wiederum als illegaler Organhandel verstanden, transplant commercialism als denkbare legale Kommerzialisierung von Organtransplantation. Tourism steht in dieser Definition also zum einen für Kommerzialisierung („commercialism“), zum anderen für illegalen Handel („trafficking“). Die Autorinnen beziehen sich also auf den semantischen Hauptaspekt Wirtschaft. Zusätzlich wird in Legalität („commercialism“) und Illegalität („trafficking“) unterschieden. Budiani-Saberi und Delmonico nehmen diese Unterscheidung von trafficking und commercialism nicht vor. Um die Uneindeutigkeit der Explikationen von transplant tourism zu illustrieren, werden die Definitionen aus der Declaration of Istanbul und die von Budiani-Saberi und Delmonico in zwei Schaubildern gegenübergestellt. 


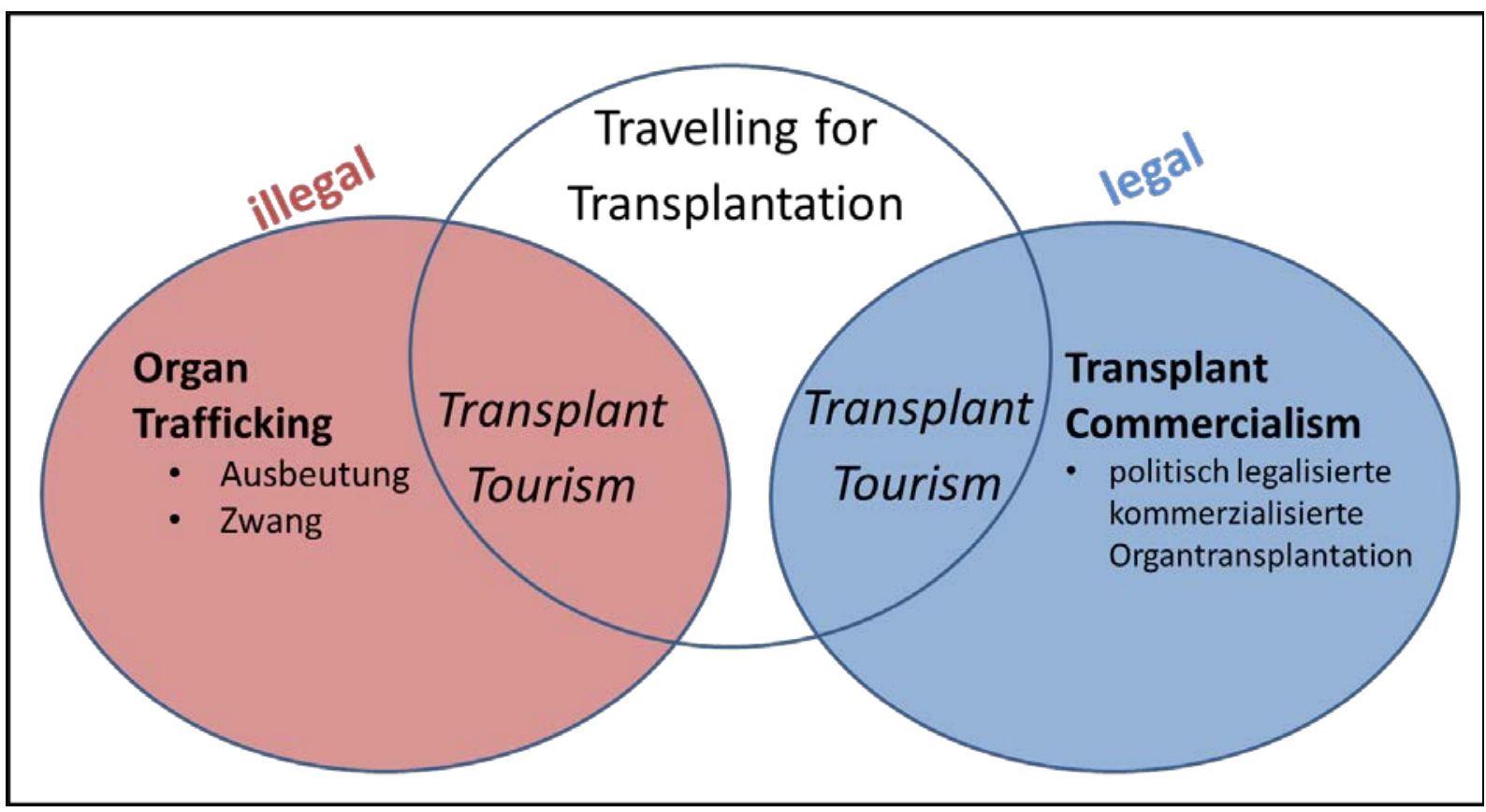

Abb. 9: Grafische Darstellung der sprachlichen Differenzierungen in der Declaration of Istanbul.

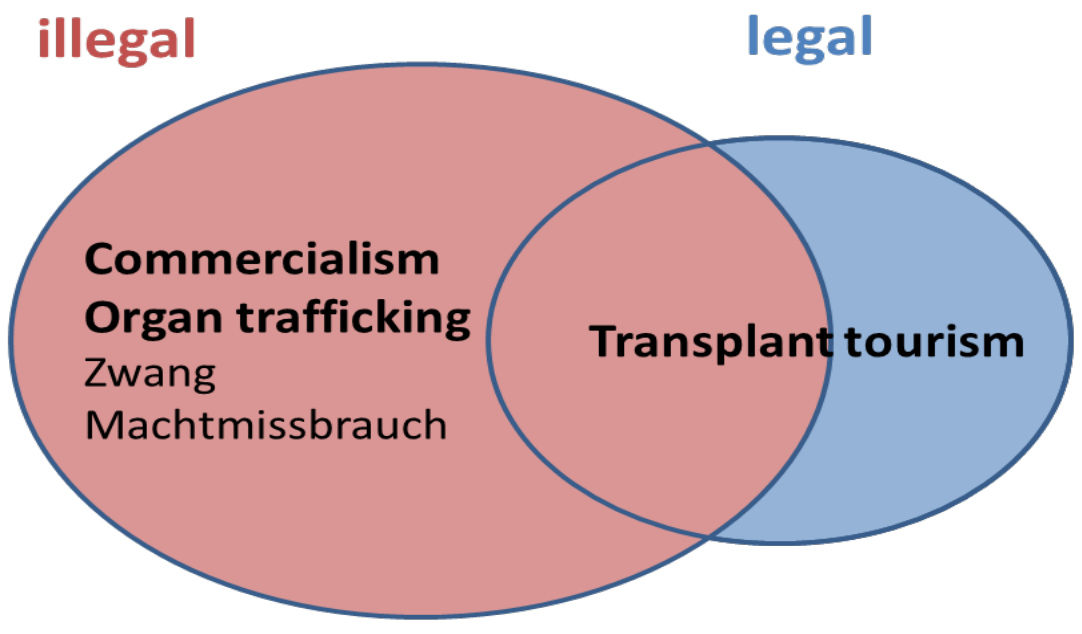

Abb. 10: Grafische Darstellung der sprachlichen Differenzierungen von Budiani-Saberi und Delmonico.

Anhand der Schaubilder wird deutlich, dass in beiden Definitionen zwischen legalem und illegalem „transplant tourism“ unterschieden wird. Budiani-Saberi und Delmonico machen diese Differenzierung explizit, indem sie feststellen, dass transplant tourism nicht immer organ trafficking 
bedeute: „Transplant tourism may be legal and appropriate“ (Budiani-Saberi und Delmonico 2008, S. 926). In der Declaration of Istanbul wird nur indirekt geäußert, dass transplant tourism auch legal sein könne: Transplant commercialism wird zunächst als „policy“ definiert, im Anschluss daran erfolgt die Definition von transplant tourism, womit sowohl illegaler Organhandel (organ trafficking) als auch legale kommerzialisierte Organtransplantationen (trasnplant commercialism) gemeint sein könne:

\footnotetext{
Travel for transplantation becomes transplant tourism if it involves organ trafficking and/or transplant commercialism or if the resources (organ, professionals and transplant centers) devoted to providing transplants to patients from outside a country undermine the country's ability to provide transplant services for its own population (The Transplantation Society und International Society of Nephrology 2008, S.2) (Hervorhebung im Original).
}

Die Autorinnen bemühen sich um eine Unterscheidung der Ausdrücke trafficking, commercialism und tourism. Diese ist jedoch verwirrend und gelingt umso weniger, als die unterschiedlichen Definitionen sich widersprechen. Noch unübersichtlicher wird die Diskussion dadurch, dass es gleichzeitig Autorinnen gibt, die weder sprachlich noch inhaltlich differenzieren; zum Beispiel setzt Kokubo in ihrer Arbeit „The interaction of the international society concerning kidney transplants" transplant tourism mit organ trafficking gleich:

On the other hand, it appears that some patients have gone overseas for kidney transplants recently. It is said that the number of patients is about a hundred. This phenomenon is not only in Japan but also in some other advanced countries and nowadays it came to be called 'Transplant Tourism' or organ trafficking (Kokubo 2009, S. 393).

Anhand der hier vorgestellten Explikationen kann gezeigt werden, dass die Autorinnen sich nicht einig darüber sind, ob mit transplant tourism legale, illegale oder alle international durchgeführten Organtransplantationen gemeint sind. Dass die Legalität beziehungsweise Illegalität in den Explikationen immer wieder eine Rolle spielt, zeigt, wie wichtig diese Fragestellung in dem als transplant tourism bezeichneten Phänomen ist. Dennoch gelingt es in den analysierten Explikationen nicht, dieses deutlich zu machen beziehungsweise sprachlich sinnvoll zu unterscheiden. Darüber hinaus kommt es zur Übertragung von Konnotationen von dem einen Sachverhalt auf den anderen. Diese Übertragung wirkt dabei in beide Richtungen: organ trafficking erwirbt die Konnotation „legaler Wirtschaftszweig“, während transplant tourism die Konnotation „illegal“ erhält. Black hat diese Übertragung in zwei Richtungen am Beispiel der Metapher „Der Mensch ist ein Wolf" erläutert: So soll durch die Metapher der Mensch als angsteinflößend dargestellt werden, gleichzeitig wirke dadurch jedoch der Wolf menschlicher. Solche Übertragungen finden auch zwischen den untergeordneten Ausdrücken wie transplant tourism und reproductive tourism statt. Etabliert sich erst einmal für transplant tourism die Konnotation „illegal“, wird die Adressatin sie auch auf andere Hyponyme übertragen, zum Beispiel auf reproductive tourism 
oder cosmetic tourism. Dabei ist in diesen Bereichen ein illegales Handeln der Reisenden zumeist nicht gegeben.

Zusammenfassend konnte am Beispiel von Explikationen zu transplant tourism gezeigt werden, dass in der ethischen Diskussion uneindeutige, teilweise miteinander unvereinbare Explikationen verwendet werden. Diese erschweren gerade im interdisziplinären Kontext die Diskussion des Themas und können zu Missverständnissen führen. Einige Autorinnen versuchen, den Ausdruck transplant tourism entgegen der ursprünglichen Semantik von Tourismus = legaler Wirtschaftszweig explizit als illegal zu definieren. Dadurch kann es zur Übertragung von Konnotationen eines Teilbereiches auf den anderen kommen, was wiederum zu Missverständnissen führt, da diese für andere Bereiche des Phänomens häufig nicht zutreffen.

\subsubsection{EINE LEITMETAPHER - VIELE AUSDRÜCKE}

Die Textanalyse hat ergeben, dass für Medizintourismus und seine Unterbegriffe eine große Anzahl unterschiedlicher Explikationen in der ethischen Debatte vorgeschlagen wurden. Am Beispiel von transplant tourism wurde gezeigt, dass die Autorinnen sich keineswegs einig sind, was der Ausdruck überhaupt bedeute. Darüber hinaus bestehen signifikante Bedeutungsunterschiede zwischen den jeweiligen Unterbegriffen, obwohl viele Autorinnen sich auf die Leitmetapher Medizintourismus berufen. Die Eindeutigkeit der Metapher Medizintourismus wird also durch eine Vielzahl an Bedeutungen der jeweiligen Hyponyme, also von der übergeordneten Metapher abgeleiteten Ausdrücke wie Transplantationstourismus, Reproduktionstourismus oder Sterbetourismus zusätzlich in Frage gestellt. Dabei beschreibt jedes dieser Hyponyme einen Teilbereich, der sich mit eigenen ethischen und rechtlichen Fragestellungen auseinandersetzt, die zu ganz unterschiedlichen Konnotationen der Metapher Tourismus führen.

An den unterschiedlichen Explikationen der Hyponyme werden unter anderem die juristischen und ethischen Fragestellungen, die in dem jeweiligen Kontext von besonderer Bedeutung sind, deutlich. So heben zum Beispiel alle Explikationen von transplant tourism hervor, dass es sich um internationale Reisen handele, während dieser Aspekt in den Explikationen von reproductive tourism nicht erwähnt wird. Während in 78\% der Explikationen von transplant tourism und in 50\% der Explikationen von reproductive tourism die Aussage getroffen wird, die Reisenden umgingen das geltende Recht ihres Wohnortes, spielt dieser Aspekt wiederum in keiner der Explikationen von medical tourism eine Rolle. Dies zeigt einmal mehr die Vielschichtigkeit des Phänomens auf. Darüber hinaus wird deutlich, wie problematisch es ist, eine so normativ wirksame Metapher auf so komplexe Phänomene anzuwenden. Und schließlich führt die Hyponymbildung zu spezifischen Konnotationen, die wiederum normativ auf andere Fachbereiche (und deren 
Hyponyme) übertragen werden können. Wenn zum Beispiel im Zusammenhang mit transplant tourism die Illegalität hervorgehoben wird, liegt die Annahme nahe, dies gelte auch für andere Hyponyme wie reproductive tourism oder dental tourism. Der Sachverhalt der Illegalität ist in den anderen Fachbereichen jedoch häufig nicht gegeben.

James Unti problematisiert diese unterschiedlichen Konnotationen der Hyponyme. Er hebt den Bedeutungsunterschied von medical tourism und transplant tourism hervor, da mit dem Ausdruck medical tourism ein neuer, vielfach beworbener Wirtschaftszweig gemeint sei, der von transplant tourism abzugrenzen sei:

\begin{abstract}
Worldwide shortages of donor organs for transplant have created global commercial opportunities in the international organ trade. Often referred to as 'transplant tourism', this form of medical tourism has little in common with the emerging industry that is being broadly promoted today. In transplant tourism, patients travel on their own to obtain organs through the organ trade or through other means that contravene the regulatory framework of their countries of origin. Many clinical and bioethical concerns surround this trade, and the unavailability of sufficient amounts of verifiable data has led to numerous superficial and often inadequate assessments of this exceedingly complex issue (Unti 2009, S. 19).
\end{abstract}

Während Unti medical tourism die Konnotation „emerging industry“ zuschreibt, versucht er, transplant tourism davon abzugrenzen. Die Konntotation eines Wirtschaftszweigs bzw. einer „Industrie“ ist jedoch in der Metapher Tourismus angelegt und entfaltet ihre Wirkung bei der Adressatin auch bei transplant tourism. Nicht umsonst verwendet Unti auch bei der Beschreibung von transplant tourism Vokabular, das dem semantischen Feld Wirtschaft angehört wie global commercial opportunities. Die Abgrenzung von transplant tourism von medical tourism gelingt also nicht, da die Rezipientin über das wirtschaftliche Vokabular auf den Gemeinplatz „Tourismus als legaler Wirtschaftszweig“ verwiesen wird und sowohl medical tourism als auch transplant tourism zum Teil in diesem Sinne verstehen wird.

Auch reproductive tourism ordnet Unti in das übergeordnete Phänomen medical tourism ein. In diesem Zusammenhang geht er vor allem auf die rechtlichen Regelungen ein, die viele Patientinnen dazu treiben, im Ausland eine reproduktionsmedizinische Behandlung durchführen zu lassen:

\footnotetext{
Reproductive outsourcing is another specialized form of medical tourism. Legal and policy limitations in many countries have created a global environment where, in a rising number of instances, individuals and couples must travel elsewhere to procure fertility procedures that are unavailable back home. Sometimes referred to as 'reproductive tourism', circumstances are created in which pregnancy is initiated in one location using the services of a fertility doctor, and parturition occurs at another (typically back home). The jargon term 'procreation vacation' has been used, and certainly, assisted conception is one of the most contentious areas of present-day medicine (Unti 2009, S. 19).
}

Unti erwähnt unterschiedliche Synonyme von reproductive tourism, die sich auf dasselbe Phänomen beziehen (reproductive tourism, procreative tourism). In diesem Abschnitt nutzt er weniger Vokabular aus dem semantischen Feld Wirtschaft, er betont vielmehr die ethische Proble- 
matik. Auch dieser Aspekt kann jedoch nicht als Kriterium zur Abgrenzung dienen, da es in allen Bereichen mit reisenden Patientinnen zu ethischen Problemen kommen kann, allerdings variieren diese abhängig vom jeweiligen Fachbereich. Während es bei Medizintourismus zum Beispiel häufig um Fragen der Verteilungsgerechtigkeit geht, stehen bei Reproduktionstourismus zumeist Fragen der Selbstbestimmung, des moralischen Status des Embryos oder des Kindeswohls im Vordergrund. Auch im als Sterbetourismus bezeichneten Phänomen geht es um spezifische ethische Aspekte des Lebensschutzes. Diesen teilweise gravierenden Unterschieden der TeilPhänomene werden die Explikationen nicht gerecht.

Im nächsten Abschnitt setzt Unti reproductive und transplant tourism zueinander in Beziehung, wobei er wiederum auf Vokabular aus dem semantischen Feld Wirtschaft zurückgreift und beides unter „industry“ zusammenfasst:

\footnotetext{
Such services [services of a fertility doctor] have many associated bioethical, legal, and other safety issues and these matters become even more complicated when travel to foreign lands is involved. Pregnancy termination presents another area with many concerns. Like transplant tourism, determining both the demands and the outcomes for these services is complicated by virtually nonexistent domestic record keeping and an unclear understanding of the size and scope of the industry (Unti 2009, S. 19) (Hervorhebungen durch M.M.).
}

Unti kritisiert zwar die Vieldeutigkeit der Ausdrücke, zählt aber sowohl transplant tourism als auch reproductive tourism zu dem als medical tourism bezeichneten Phänomen. Darüber hinaus nutzt er den Tourismus-Ausdruck selbst in dem Sinne des Hauptaspektes Wirtschaftszweig: Er spricht bei „medical tourism“, „transplant tourism“ und „reproductive tourism“ gleichermaßen von einer „industry“. Unti versucht, die unterschiedlichen Teil-Phänomene voneinander abzugrenzen, dies gelingt jedoch allenfalls eingeschränkt, da über das Vokabular aus dem semantischen Feld Wirtschaft die Adressatin immer wieder auf den Gemeinplatz „Tourismus als legaler Wirtschaftszweig" gebracht wird.

Zusammenfassend konnte gezeigt werden, dass die definitorischen Aussagen eines Hyponyms nicht ohne weiteres auf die Leitmetapher Medizintourismus oder weitere Hyponyme übertragbar sind, obwohl in den Texten und Explikationen explizit der Zusammenhang zwischen Hyponym und Leitmetapher benannt wird. Sowohl die Varianz der Explikationen als auch die Vielzahl an Hyponymen mit jeweils unterschiedlichen impliziten Botschaften dienen nicht der Sachlichkeit der Diskussion. Außerdem konnte gezeigt werden, dass die Bildung und Verwendung unterschiedlicher Hyponyme, die sich auf die Leitmetapher Medizintourismus beziehen, problematisch ist, da je nach diskutiertem Fachbereich und Hyponym die ethischen Fragestellungen und damit auch die Konnotation des Ausdrucks variieren. Es kommt zur Bildung und Übertragung neuer Konnotationen, woraus Missverständnisse resultieren. Gleichzeitig können Übertragungen von 
Konnotationen von einem Hyponym auf ein anderes beobachtet werden, die inhaltlich nicht immer richtig sind. Auf diesen Punkt wird im Folgenden noch ausführlicher eingegangen.

\subsection{ORTSWECHSEL - FreIZEIT - WirTSCHAFT: SEMANTISCHE FELDER VON TOURISMUS IM ZUSAMMENHANG MIT MEDIZINTOURISMUS}

Die Auswertung der 45 Texte aus der ethischen Diskussion belegt deutlich, dass die Semantik von Tourismus in den Texten, die den Ausdruck Medizintourismus oder eines der Hyponyme verwenden, eine wichtige Rolle spielt. Die drei semantischen Hauptaspekte Ortswechsel, Freizeit und Wirtschaft spiegeln sich in dem Vokabular der Texte wider. Im Folgenden sollen für jedes semantische Feld Beispiele aus dem Korpus diskutiert werden. Anschließend werden die semantischen Aspekte im Zusammenhang mit den unterschiedlichen Funktionen einer Metapher diskutiert.

Dem semantischen Feld Ortswechsel können insgesamt 392 Zitate zugeordnet werden. Das entspricht etwa 9 Zitaten pro Text. Gleichzeitig wird das semantische Feld Ortswechsel als einziger der drei Hauptaspekte von Tourismus in allen Explikationen genannt. Dabei wird das semantische Feld aber auf unterschiedliche Zusammenhänge angewendet. Während sich einige Autorinnen ausschließlich auf Patientinnen beziehen, die für eine medizinische Behandlung ins Ausland gehen, schließen andere Personal mit ein, das außerhalb des eigenen Wohnortes arbeitet. Als Beispiele für die Anwendung des semantischen Feldes Ortswechsel können folgende Textstellen herangezogen werden:

„Transplant 'tourism' typically refers to the practice of traveling outside the country of residence to obtain organ transplantation“ (Gill et al. 2008, S. 1820); „On the other hand, it appears that some patients have gone overseas for kidney transplants recently“ (Kokubo 2009, S. 393); „More recently, citizens of developed countries, including the USA and Europe, have chosen to travel to less developed parts of the world for medical treatment, thereby bypassing services in their own communities“ (Leahy 2008, S. 260); „It has focused attention on a phenomenon known as stemcell tourism, in which desperately ill patients and their families go to clinics all over the world in the hope of finding at least an improvement in their condition, if not a cure" (MacReady 2009, S. 317); „Second, some American patients must go abroad to get medically accepted treatments that are not available at home due to political prohibition" (Bauer 2009, S. 37) (Hervorhebungen durch M.M.).

Auch das semantische Feld Wirtschaft wird in unterschiedlichen Zusammenhängen bedient. Budiani-Saberi und Delmonico versuchen, ihrer Kritik an den kommerziellen Strukturen im Bereich der Organtransplantation durch die Verwendung von Vokabular aus dem semantischen 
Feld Wirtschaft Ausdruck zu verleihen: „The extent of organ sales from commercial living donors (CLDs) or vendors has now become evident" (Budiani-Saberi und Delmonico 2008, S. 925) (Hervorhebungen durch M.M.). Carabello beschreibt tourismuswirtschaftliche Strukturen im Zusammenhang mit medical tourism und greift auf entsprechendes Vokabular zurück:

Patients who are not familiar with specific medical facilities abroad can coordinate their treatment through medical travel intermediaries. These services work like specialized travel agents. They investigate healthcare providers to ensure quality and screen customers to assess those who are physically well enough to travel (Carabello 2008, S. 293) (Hervorhebungen durch M.M.).

Carabello greift in deskriptiver Absicht auf Vokabular aus dem wirtschaftlichen Bereich zurück. Gleichzeitig wird die Metapher durch die schlüssige Anwendung bestätigt. Es handelt sich um eine affirmative Verwendungsweise. Diese wirkt epistemisch normativ, da sie die Wahrnehmung bestimmter Strukturen des Phänomens erwirkt. Budiani-Saberi und Delmonico hingegen wollen durch Verwendung von Vokabular aus dem wirtschaftlichen Bereich die kommerziellen Umstände kritisieren, das heißt sie verwenden die Metapher moralisch normativ. Auf diese unterschiedlichen Verwendungsweisen der Metapher wird später ausführlicher eingegangen.

Das semantische Feld Freizeit wird in den Texten deutlich seltener bedient als die semantischen Felder Ortswechsel und Wirtschaft. Es wird in 2 von 27 Explikationen genannt, so zum Beispiel von Kantor, die medical tourism unter anderem als „[activity] where consumers take exotic vacations and combine them with surgery" charakterisiert (Kantor 2008, S. 1-2) (Hervorhebung durch M.M.). Im Textkorpus zeigt sich das semantische Feld Freizeit beispielsweise in dem Zitat von Mitka: „To further attract patients, some of these offshore medical centers often employ surgeons who are US trained and board certified, highlight their status as approved by an internationally accepted accreditation organization, and include airfare plus a stay at a luxury hotel" oder in folgendem Zitat von Unti: „Imagine a patient leaving the U.S. along with a family member or other companion and flying off to some exotic locale halfway around the world to receive a needed surgical treatment" (Mitka 2009, S. 1519; Unti 2009, S. 19) (Hervorhebungen durch M.M.). Auch der Bioethiker Leigh Turner verwendet in seinem Text Vokabular aus dem semantischen Feld Freizeit: „The optimistic view of this phenomenon is that patients are taking inexpensive ,dental vacations' in exotic locales. The skeptical view is that patients risk receiving inferior care in regions with lower regulatory standards and limited oversight of dental clinics“ (Turner 2009, S. 118) (Hervorhebung durch M.M.). Die Intentionen der Autorinnen variieren in der Anwendung dieses semantischen Feldes. Während einige tatsächlich Parallelen zwischen den Phänomenen Tourismus zu medizinischen Zwecken reisenden Personen aufzeigen möchten, verwenden andere das Vokabular, um diese Umstände zu kritisieren. Auf die unterschiedlichen Verwendungsweisen wird später noch näher eingegangen. 
Die semantischen Felder werden in den Texten unterschiedlich stark berücksichtigt. Auffällig ist dabei eine Diskrepanz in der Häufigkeitsverteilung der semantischen Felder in den Explikationen einerseits und dem gesamten Textkorpus andererseits: Während in den Explikationen das semantische Feld Ortswechsel am häufigsten angeführt wird, ist dies auf das gesamte Textkorpus bezogen das semantische Feld Wirtschaft. Zur Verdeutlichung wird die entsprechende Abbildung noch einmal angeführt:

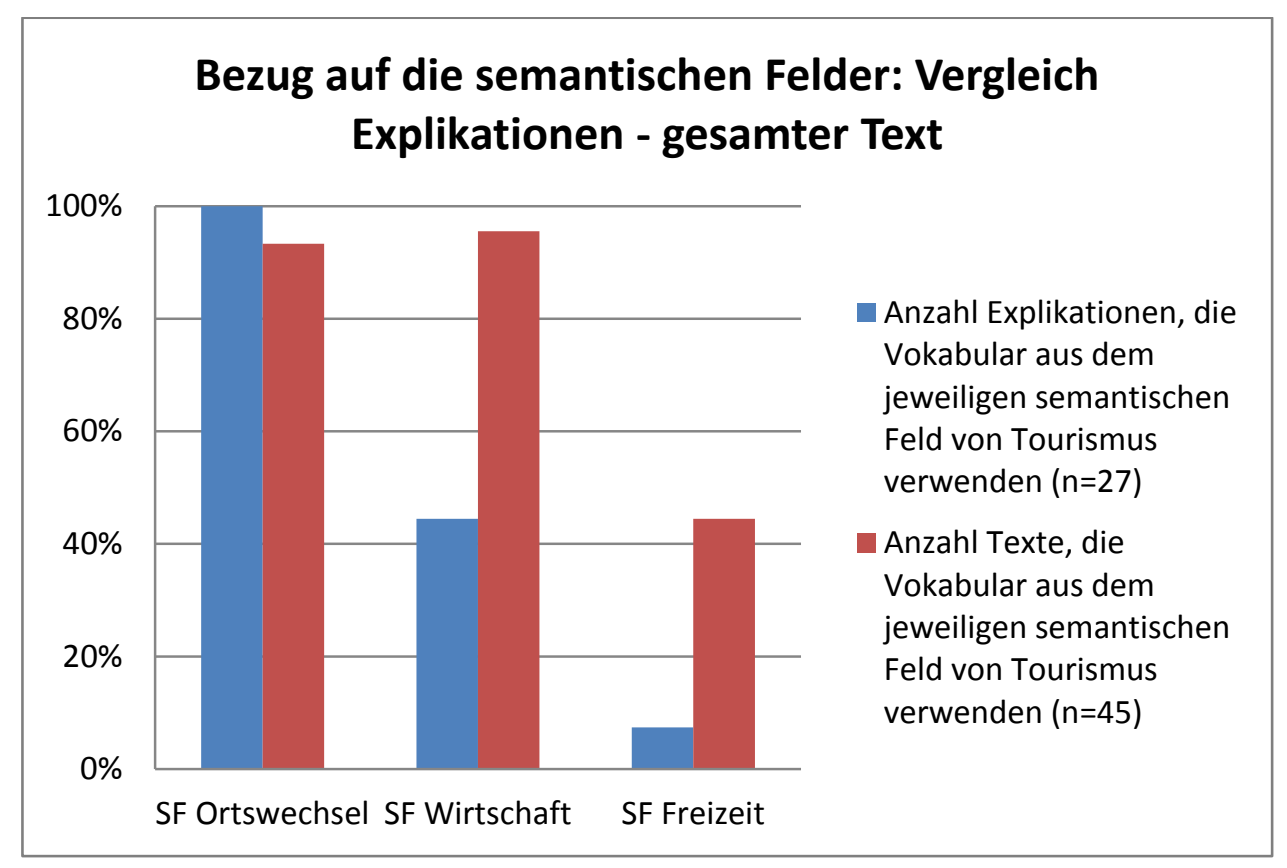

Abb. 6: Gegenüberstellung der Anwendung der semantischen Felder in den Explikationen und im gesamten Textkorpus.

Diese Diskrepanz der Häufigkeitsverteilung der unterschiedlichen semantischen Felder in den Explikationen und im gesamten Korpus macht deutlich, dass die Konnotationen eines Ausdrucks nicht nur durch Explikationen bestimmt und daher durch diese nur bedingt zu beeinflussen sind.

Die Ergebnisse der Textanalyse zeigen deutlich, dass die Semantik von Tourismus in Texten, die Medizintourismus oder ein Hyponym verwenden, eine Rolle spielt. Das aus dem Zusammenhang von Tourismus bekannte Vokabular wird dazu genutzt, das Phänomen der transnationalen Medizin zu diskutieren. Dass die semantischen Felder von Tourismus tatsächlich zur Anwendung kommen, bestätigt den Ausdruck Medizintourismus als Metapher.

\subsection{Die Leistungen der Metapher Medizintourismus}

Angelehnt an die Metapherntheorie von Kovács sollen in diesem Kapitel die unterschiedlichen Funktionen der Metapher Medizintourismus und der dazugehörigen Hyponyme vorgestellt werden. Ich entferne mich dabei insofern von der Theorie Kovács', als ich nicht vier Leistungen un- 
terscheide (illustrativ, innovativ, konstitutiv, normativ), sondern nur die drei Funktionen illustrativ, innovativ und konstitutiv, die jede für sich wiederum normatives Potential haben. Kovács legt dar, dass die „Normativität der Metapher [...] also keine moralische, sondern eine epistemische" sei (Kovács 2009, S. 61). Im Gegensatz dazu möchte ich aufzeigen, dass die Metapher Medizintourismus sowohl epistemisch als auch moralisch normativ wirkt. Während sich die epistemische Normativität der Funktionen auf die Wahrnehmung und Konzeptionalisierung des Phänomens bezieht, ist mit der moralischen Normativität eine Wertung des Sachverhaltes gemeint. Jede der oben genannten Funktionen (illustrativ, innovativ, konstitutiv) kann für sich genommen sowohl epistemisch als auch moralisch normativ wirken.

In der Praxis sind diese Formen von Normativität nicht immer scharf voneinander abzugrenzen. Häufig folgt aus der epistemischen direkt oder indirekt eine moralische Normativität. Die Unterscheidung ist aber dennoch hilfreich, um zu einem besseren Verständnis der Wirkungsweise der Metapher Medizintourismus beizutragen.

Auch andere Autorinnen unterscheiden die Wirkungsweisen einer Metapher und beschreiben deren Normativität. Warren Shibles und Barbara Maier zeigen, wie eine Metapher einerseits täuschen, andererseits die Wahrnehmung von Zusammenhängen begünstigen kann: „When metaphor becomes literally it turns metapher into myth, delusion, and dogma. Perspectival thinking is lost. If we unknowingly take a metaphor literally, it is a fallacy. If we deliberately take it as a way to create insight, it can be a significant tool for inquiry" (Shibles und Maier 2011, S. 3).

Die Autorinnen legen dar, eine Metapher könne zur Täuschung und sogar zum Dogma werden, wenn sie im Diskurs wörtlich gebraucht werde. Dies entspricht bei Kovács der konstitutiven Funktion: Das Verständnis eines Phänomens wird durch die Verwendung einer Metapher auf bestimmte Denkstrukturen festgelegt. Gleichzeitig beschreiben Shibles und Maier die Metapher als ein Instrument, das dazu geeignet ist, Verständnis für bestimmte Strukturen zu erzeugen. Diese Wirkweise entspricht nach Kovács der innovativen Funktion. Die Adressatin wird durch die Metapher angeregt, bestimmte Strukturen überhaupt erst zu erkennen. Dies entspricht zum Beispiel einer epistemischen Normativität. Da es jedoch über die konstitutive Funktion möglich ist, durch die Metapher Täuschungen oder sogar Dogmen zu begünstigen, handelt es sich hier nicht mehr bloß um eine epistemische, sondern auch um eine moralische Normativität. Das Zitat von Shibles und Maier zeigt, wie fließend der Übergang von der epistemischen zur moralischen Normativität sein kann.

Im Folgenden sollen die unterschiedlichen Funktionen einzeln besprochen werden, um die vielfältige Wirkungsweise der Metapher Medizintourismus darzustellen. Es werden die illustrative, 
innovative und konstitutive Funktion anhand der Ergebnisse der Textanalyse diskutiert. Die normativen Verwendungsweisen werden im Anschluss daran ausführlich erörtert.

\subsubsection{DIE ILLUSTRATIVE LEISTUNG: DER REISEASPEKT}

Die illustrative Funktion einer Metapher basiert auf Parallelen zwischen dem neuen Phänomen und dem alten Sachverhalt, die sich der Adressatin unmittelbar erschließen. Die Metapher dient der Erklärung jener Strukturen, die sich in beiden Phänomenen ähneln und illustriert dadurch einen neuen Sachverhalt, für den bis dahin keine andere Bezeichnung vorlag. Der Ausdruck Tourismus fordert also die Adressatin dazu auf, nach Strukturen, die sie aus dem Sachverhalt „Tourismus" kennt, in dem unbekannten Phänomen zu suchen. Dabei wird die Adressatin sich zunächst auf die als bekannt vorauszusetzenden Gemeinplätze aus der Semantik des alten Sachverhaltes beziehen. Die ermittelten semantischen Hauptaspekte von Tourismus sind Ortswechsel, Freizeit und Wirtschaft. Im Zusammenhang mit Medizintourismus kann die illustrative Funktion der Metapher an dem Hauptaspekt Ortswechsel gezeigt werden: Der Reiseaspekt stellt die einzige Analogie aller Explikationen des Textkorpus dar und entspricht dabei gleichzeitig einem semantischen Hauptaspekt der vorgeschlagenen Metapher Tourismus.

Die Metapher Medizintourismus ist offensichtlich gut dazu geeignet, Reisebewegungen in dem neuen Sachverhalt zu illustrieren, die Illustration lässt jedoch offen, auf wen oder was genau sich diese Analogie bezieht. Dies gibt Raum für eine weite Spanne von Interpretationen, die von Patientinnen über Transplantationsexpertinnen und Medizinstudierenden bis zu Organen oder Eizellen reicht und die in der Diskussion zu gravierenden Missverständnissen führen kann.

Die illustrative Funktion birgt in sich eine epistemische Normativität, da sie die Hervorhebung bestimmter Aspekte im neuen Phänomen erwirkt und so die Wahrnehmung der Adressatin beeinflusst. Der in diesem Fall hervorgehobene Reiseaspekt steht dabei jedoch nicht isoliert, sondern ist im semantischen Zusammenhang mit anderen Aspekten von Tourismus zu sehen. Dies wird in der Definition des Brockhaus' von Tourismus deutlich. Der Brockhaus stellt fest, dass es „im alltäglichen Sprachgebrauch [...] ferner zu einer Gleichsetzung von Urlaub und Tourismus“ komme (Brockhaus 2009). Urlaub wiederum vereint zwei der semantischen Hauptfelder von Tourismus: Urlaub lässt sich sowohl dem Aspekt Ortswechsel als auch Freizeit zuordnen. Primär wird der Rezipientin also suggeriert, es handele sich bei medizintouristischen Reisen um Urlaubsaktivitäten. Das wiederum heißt, die Metapher wirkt über die illustrative Funktion und epistemische Normativität hinaus auch moralisch normativ, und zwar unabhängig von den tatsächlichen Zusammenhängen. Über die Assoziationskette Tourismus - Reise - Urlaub kann eine Adressatin schnell zu der Bewertung der Situation der Reisenden kommen, diese befänden sich 
auf einer allgemein als angenehm bewerteten Urlaubsreise, anstatt auf der Suche nach einer häufig durch Leid verursachten und mit Strapazen verbundenen medizinischen Behandlung. Denn dass der Freizeitaspekt in der Realität der reisenden Patientinnen eine untergeordnete Rolle spielt, zeigt sich nur bei eingehender Beschäftigung mit der Thematik. Bei dem Freizeitaspekt handelt es sich um eine Konnotation, die dem Ausdruck Tourismus zugeordnet wird, in der Realität der Reisenden im Zusammenhang mit medizinischen Behandlungen jedoch kaum in dem Maße, wie durch den Ausdruck suggeriert wird, eine Rolle spielt. Auf diese moralisch normative Wirkung der Metapher wird später noch ausführlicher eingegangen, im Folgenden soll zunächst die innovative Leistung der Metapher diskutiert werden.

\subsubsection{DIE INNOVATIVE LEISTUNG: DER WIRTSCHAFTSASPEKT}

Spricht man von der innovativen Leistung einer Metapher, meint man die Erkenntnis des neuen Phänomens entlang der semantischen Strukturen des alten Ausdrucks über die nächstliegende Analogie (in diesem Fall den Reiseaspekt) hinaus. Die Metapher regt die Kreativität an und fordert die Adressatin dazu auf, weitere Ähnlichkeiten im Hinblick auf den durch die Metapher vorgeschlagenen Deutungsrahmen zu entdecken. Je interdisziplinärer ein Diskurs ist, desto kreativer das Weiterentwicklungs-Potential einer Metapher, da Personen zusammenkommen, die unterschiedliche Begriffe von dem Ausdruck Tourismus haben, mit jeweils unterschiedlicher Gewichtung der semantischen Aspekte des Ausdrucks.

So werden zum Beispiel durch die Metapher Medizintourismus wirtschaftliches Potential und wirtschaftliche Strukturen im neuen Sachverhalt aufgezeigt. Dies gelingt, da der Wirtschaftsaspekt einen der drei semantischen Hauptaspekte von Tourismus darstellt.

Die amerikanischen Wirtschaftswissenschaftler Michael Horowitz und Jeffrey Rosensweig stellen in ihrem Artikel über das amerikanische Gesundheitswesen im globalen Marktzusammenhang fest: „medical tourism is market driven“. Sie benutzen in ihrem Artikel eine Sprache, die von wirtschaftlichen Ausdrücken geprägt ist, einige Beispiele dafür sind „export health care“, „to purchase health care“ oder „medical tourism marketplace“. Außerdem erwähnen sie Agenturen, die Patientinnen bei der Vermittlung an Krankenhäuser im Ausland helfen und Unterkunft, Reise, Zahlungsverkehr bis hin zu Ausflügen zu bekannten Sehenswürdigkeiten oder Safaris organisieren (Horowitz und Rosensweig 2007, S. 24-26).

Auch in dem von mir analysierten Textmaterial lässt sich eine Vielzahl ähnlicher Beispiele finden; zur Verdeutlichung sollen hier nur einige wenige aufgeführt werden: 
Der Gesundheitsökonom Jeffrey C. Bauer schreibt in seinem Artikel „Medical tourism: wave of the future in a world of hurt?“: „The international marketplace could be rewarding to American providers, payers, vendors, and other health-care stakeholders that can efficiently and effectively respond to the current crisis“ (Bauer 2009, S. 36) (Hervorhebungen durch M.M.). Laura Carabello verwendet ebenso Vokabular aus dem wirtschaftlichen Bereich:

\footnotetext{
Researchers report that one of the most promising solutions for our nation's soaring healthcare costs may be found in sending our patients outside the country for care, largely the result of healthcare spending per capita growing at twice the rate of growth of national income, an unsustainable path that is on course to crowd out all other consumption (Carabello 2008, S. 291) (Hervorhebungen durch M.M.).
}

Da Wirtschaftswissenschaftlerinnen in der Semantik von Tourismus den Wirtschaftsaspekt deutlicher wahrnehmen als Laien, regt die Metapher sie dazu an, das Phänomen entlang dieser Strukturen zu verstehen. Die Metapher Medizintourismus ist gut dazu geeignet, die wirtschaftlichen Aspekte des neuen Phänomens analog zum Sachverhalt Tourismus zu erfassen. So ist zum Beispiel bei medizinischen Behandlungen im Ausland im Regelfall die Patientin die Kostenträgerin, nicht etwa die Krankenversicherung. Diese kreative Weiterentwicklung des Ausdrucks Medizintourismus durch Wirtschaftswissenschaftlerinnen wird durch die Semantik des Ausdrucks Tourismus ermöglicht und spiegelt sich in der Nutzung des Vokabulars wider: Von den insgesamt 584 Zitaten mit semantischem Feld Wirtschaft entfallen 188 Zitate auf Texte von Autorinnen mit wirtschaftlichem Hintergrund. Das ergibt im Schnitt 31 Zitate pro Text, wenn die Autorin einen wirtschaftlichen Hintergrund hat, gefolgt von 15 Zitaten bei Autorinnen mit ethischem Hintergrund und 13 Zitaten in Texten von Autorinnen mit anthropologischem Hintergrund.

An dem Beispiel des Wirtschaftsaspektes lässt sich die innovative Funktion der Metapher Medizintourismus zeigen: Es wird der Versuch unternommen, weitere semantische Aspekte als die offensichtlichste Analogie (in diesem Fall der Reiseaspekt) auf den neuen Sachverhalt zu übertragen. In den Explikationen wird der Wirtschaftsaspekt nur selten ausdrücklich angeführt, im gesamten Korpus ist das semantische Feld Wirtschaft hingegen das am häufigsten angeführte. Diese Diskrepanz macht die innovative Funktion deutlich: Die Metapher ermöglicht über ihre ursprüngliche Semantik, dass neue, im ersten Augenblick nicht offensichtliche Parallelen zwischen dem alten und dem neuen Sachverhalt erfasst werden.

Die innovative Funktion entfaltet in erster Linie eine epistemische Normativität, da sie das Erkennen bestimmter Strukturen erwirkt. Da wirtschaftliche Aspekte bei Reisen zu medizinischen Zwecken beziehungsweise im Phänomen der transnationalen Medizin tatsächlich vorhanden sind, gelingt dies und wirkt schlüssig. Die moralische Normativität entfaltet sich zum Beispiel dann, wenn die Diskussion über eine angemessene Gesundheitsversorgung implizit auf ein freies marktwirtschaftliches System festgeschrieben wird und somit Alternativen, zum Beispiel eine 
Gesundheitsversorgung, die auf dem Solidaritätsprinzip beruht, ausgeschlossen werden. Dies entspricht der konstitutiven Leistung, die im folgenden Kapitel ausführlich besprochen wird.

\subsubsection{Die Konstitutive Leistung: Der Freizeitaspekt/Die PERSPEKTIVE DER Pati-} ENTINNEN

Die Autorinnen mit wirtschaftswissenschaftlichem Hintergrund bedienen sich der TourismusMetapher, um die wirtschaftlichen Ähnlichkeiten zwischen dem neuen Sachverhalt und dem Deutungsrahmen Tourismus in tatsächlicher Analogie zu dem Wirtschaftszweig Tourismus zu diskutieren. Über die innovative Funktion können die wirtschaftlichen Aspekte des Phänomens erkannt und erklärt werden. In der affirmativen Anwendung durch die Wirtschaftswissenschaftlerinnen, aber auch durch andere Beteiligte wie Reiseagenturen zeigt sich die konstitutive Funktion. Der neue Sachverhalt wird als Wirtschaftszweig zunächst kreativ interpretiert, später darauf festgelegt und als solcher verhandelt.

Laut Kovács zeigt sich die konstitutive Funktion einer Metapher am Metaphernsystem. Aus der den Deutungsrahmen vorgebenden Leitmetapher würden weitere inhaltliche Übertragungen folgen. Diese würden sich an weiteren, dem Deutungsrahmen zugehörigen Metaphern zeigen. Auf die Metapher Medizintourismus bezogen, fordert die konstitutive Leistung demnach dazu auf, in dem Metaphernsystem Medizintourismus Touristinnen zu identifizieren, die in Hotels unterkommen oder weitere touristische Aktivitäten ausüben, Sehenswürdigkeiten aufsuchen oder auf Safari gehen. Gleichzeitig wird die Rezipientin durch die konstitutive Funktion daran gehindert, über die Metapher hinausgehende Strukturen in dem neuen Sachverhalt zu erkennen.

Horowitz und Rosensweig beschreiben in diesem Sinne zum Beispiel Agenturen, die nicht nur die Vermittlung von Patientinnen an Krankenhäuser übernehmen, sondern zusätzlich Ausflüge zu bekannten Sehenswürdigkeiten organisieren (Horowitz und Rosensweig 2007). Dabei wird nicht nur die touristische Aktivität als Parallele verhandelt, auch die Agentur, die diese vermittelt, wird in Analogie zu Reiseagenturen verstanden. Tatsächlich gibt es Reiseagenturen, die ihr Spektrum erweitert haben und spezielle „medizintouristische Reisen“ anbieten. Hierbei handelt es sich um eine affirmative Interpretation der Reiseagenturen selbst.

Kristen Smith benennt weitere Strukturen, die mit Hilfe des Metaphernsystems zu erkennen sind, z. B. die Überschneidungen von Krankenhaus und Hotel. Dabei handelt es sich zwar nicht um eine metaphorische Beschreibung, sondern um eine Annäherung der Bedeutung der jeweiligen Einrichtungen, dennoch wird deutlich, dass durch den Tourismus-Ausdruck diese Denkrichtung unterstützt wird: 
The uncanny parallels of the modern, five-star corporate hospital offering health services for medical travellers, with the corporate five-star hotel industry do not arise by accident. Hospital websites advertise their international patient administrators as ,concierges' and promote hotel style accommodation provided for international patients, some even replete with kitchenettes, adjoining bedrooms, gymnasiums, swimming pools and wall mounted, wide-screen televisions. These commercial, touristic elements are aggressively promoted by many hospitals participating in the trade (Smith 2012, S. 3) (Hervorhebungen durch M.M.).

Auch bei Gill et al. finden sich in dem Aufsatz „Transplant tourism in the United States: a singlecenter experience" Passagen, in denen Vokabular verwendet wird, die Tourismus als übergeordnetes Metaphernsystem sichtbar machen: Während Patientinnen, die im Ausland ein Transplantat bekommen haben, als „tourists“ beschrieben werden, werden Patientinnen aus der Kontrollgruppe, die an einer amerikanischen Klinik transplantiert wurden, als „patients“ bezeichnet. Dies geschieht systematisch und absichtlich, denn diejenigen Patientinnen, die im Ausland ein Transplantat erhalten haben, werden zu Beginn des Textes als „tourists“ definiert:

We used the term ,tourist' to describe residents of the United States who underwent transplantation outside the United States and then returned to the United States for follow-up care, keeping in mind that we did not know all of the circumstances surrounding the recipients' reason to pursue a transplant abroad (Gill et al. 2008, S. 1820).

Daraufhin verwenden die Autorinnen durchgängig tourist anstatt patient oder transplant tourist. Die Patientinnen der Kontrollgruppe aus dem Inland werden als patients bezeichnet. Es kommt zu Aussagen wie: „Propensity scores were calculated using logistic regression and were used to match the 33 tourists to 66 patients who underwent transplantation at UCLA“ oder: „This supports recent data by Merion et al. that indicated a tripling in the number of identified tourists who returned to the United States from 2000 to 2006" (Gill et al. 2008, S. 1821, S. 1825) (Hervorhebungen durch M.M.).

Die Verwendung von tourist anstatt patient kann dabei nicht nur der Verkürzung und damit besseren Lesbarkeit des Textes geschuldet sein, da die Kontrollgruppe aus dem Inland als „patients receiving transplantation at UCLA“ ausführlich bezeichnet wird. Eine sprachlich entsprechende Bezeichnung für die andere Gruppe wäre zum Beispiel patients receiving transplantation outside the USA. An diesem Beispiel zeigt sich die Konstituierung des Sachverhaltes: aus Patientinnen werden Touristinnen.

Die Verwendung des Ausdrucks tourist erwirkt aber nicht nur eine Konstituierung des Sachverhaltes, sondern auch eine Hervorhebung der touristischen Aktivitäten gegenüber der Eigenschaft der Betroffenen, Patientin zu sein. Hier zeigt sich die epistemische Normativität der Metapher: Die medizinische Behandlung, die in der Leitmetapher Medizintourismus noch berücksichtigt ist, taucht in der Bezeichnung tourist überhaupt nicht mehr auf und rückt auch in der Wahrnehmung der Rezipientin in den Hintergrund. 
Die konstitutive Wirkung einer Metapher besteht darin, dass der neue Sachverhalt auf den Deutungsrahmen festgelegt wird. Dies bedeutet in Bezug auf die Metapher Medizintourismus, dass zum Beispiel Patientinnen auf die Touristinnenrolle festgelegt werden und eine andere Darstellung des Sachverhaltes durch den Gebrauch der Metapher verhindert wird. Durch die Verwendung des Ausdrucks tourist kommt es zur Hervorhebung bestimmter Aspekte beziehungsweise Aktivitäten der Betroffenen. Darüber hinaus werden über die Nicht-Verwendung des Ausdrucks patient die damit verbundenen Aspekte für diese bestimmte Gruppe ausgeblendet. Dies schließt eine Bewertung darüber mit ein, was für den Sachverhalt als relevant anzusehen ist.

Dieser Effekt lässt sich gut anhand jener Bezeichnungen verdeutlichen, die in der Diskussion bereits vorgeschlagen wurden, um den Ausdruck reproductive tourism zu ersetzen. So plädierte Matorras 2005 für den Ausdruck reproductive exile. Der Ausdruck exile verändert den Blickwinkel der Adressatin auf die Lage der Patientinnen. So wird zum Beispiel die Freiwilligkeit, eine Reise aus reproduktionsmedizinischen Gründen zu unternehmen, infrage gestellt und vielmehr ein äußerer Zwang suggeriert (Matorras 2005). An diesem Beispiel wird deutlich, inwiefern die Wahl einer bestimmten Metapher die Wahrnehmung und damit auch die Bewertung eines Phänomens beeinflusst.

Der Ausdruck Tourismus betont also die Reiseaktivität der Betroffenen, lässt jedoch den Leidensdruck der Patientinnen außer Acht. Gleichzeitig nutzen einige Autorinnen das semantische Feld Freizeit, um die positiv besetzten Freizeitaspekte der zu medizinischen Zwecken durchgeführten Reise hervorzuheben. So beschreibt zum Beispiel Pafford in dem Artikel „The Third Wave - Medical Tourism in the 21st Century“ die Situation einer Patientin, die für eine BypassOperation aus den USA nach Thailand reist, folgendermaßen:

\begin{abstract}
The next day she arrives at the Suvarnabhumi International Terminal in Bangkok, Thailand. A waiting car takes them to the Bumrungrad Hospital where she is given a private room with luxury accommodations for her husband nearby, one-on-one care, and specially cooked meals. In the morning she undergoes surgery and, after a few days, continues her recovery at a four-star hotel next to the hospital-complete with assisted tours to nearby sights. When stable, she returns home to Kansas with pictures from her excursions, a taste for Thai food, and follow-up care with her local cardiologist (Pafford 2009, S. 810) (Hervorhebungen durch M.M.).
\end{abstract}

Turner geht darüber hinaus auf die Rolle der Vermittlungsagenturen ein: „Dental tourism companies advertise 'all-inclusive' travel packages that include dental procedures, hotel room reservations, side trips to tourist attractions and airline tickets“ (Turner 2009, S. 117) (Hervorhebungen durch M.M.).

Auch Gupta und Unti verwenden Vokabular aus dem semantischen Feld Freizeit: „Medical tourists usually get a package deal that includes flights, hotels, treatment and, often, a post-operative 
vacation“ (Gupta 2008, S. 4) (Hervorhebungen durch M.M.). „Following treatment, the 'tourist" experiences personal medical attention in a luxurious setting with first-class accommodations and subsequently has the chance to enjoy a vacation for a short while before returning home“ (Unti 2009, S. 19) (Hervorhebungen durch M.M.). Durch solche Betonungen des Freizeitaspektes wird die Vorstellung von Patientinnen als Touristinnen festgeschrieben.

Die Anwendung des semantischen Feldes Freizeit setzt sich jedoch nicht in gleichem Maße durch, wie die Anwendung des semantischen Feldes Wirtschaft. Dies wird anhand der unterschiedlichen Häufigkeitsverteilung der semantischen Felder deutlich: Während das semantische Feld Wirtschaft in 584 Zitaten angewandt wird, wird das semantische Feld Freizeit in nur 63 Zitaten bedient. Zwar kann die konstitutive Funktion durch das semantische Feld Freizeit gezeigt werden, sie ist aber im Vergleich zur innovativen Funktion am Beispiel des Wirtschaftsaspekts weniger deutlich.

Die konstitutive Funktion entfaltet ihre Wirkung jedoch nicht nur durch die Festschreibung der Vorstellung von Patientinnen als Freizeittouristinnen. Durch die Verwendung der Metapher wird es zugleich schwieriger, das Leid der Patientinnen zu erfassen. Wenn die Sichtweise auf das Phänomen als Tourismus festgelegt ist, wird es erschwert, weitere Sachverhalte in den Blick zu nehmen, die außerhalb der Semantik von Tourismus liegen. Diese Wirkung kann anhand der Berücksichtigung des Leidaspektes im Textmaterial gezeigt werden, wie ich im nächsten Kapitel zeigen werde.

\subsubsection{DER LEIDASPEKT: VON DER INNOVATIVEN ZUR KONSTITUTIVEN LEISTUNG}

Anhand des Leidaspektes sollte die konstitutive Funktion der Metapher Medizintourismus überprüft werden. Überraschend fiel auf, dass das semantische Feld Leid insgesamt häufiger als das semantische Feld Freizeit bedient wird. Der Analyse des Leidaspektes lag schließlich die Annahme zugrunde, die Metapher liefere der Adressatin kein Konzept, um die häufig verzweifelte Lage der Patientinnen berücksichtigen zu können.

Dieses zunächst unerwartete Ergebnis ist jedoch darauf zurückzuführen, dass Leid in den Texten vorrangig im Zusammenhang mit der betroffenen Bevölkerung in den Zielländern erwähnt wird. Biggins et al. schreiben in ihrem Artikel über Transplantation von Gefangenen in China an Reisende und beschreiben Folgendes: „These reports indicate that over 95\% of organ donors in China are prisoners. Prisoners, particularly those slated for execution, are a vulnerable population particularly susceptible to coercion" (Biggins et al. 2009, S. 831). Auch Budiani-Saberi und Delmonico schreiben: „The source of their allografts is mainly from the poor and vulnerable in the developing world. These vendors or commercial living donors resort to an organ sale be- 
cause they have virtually no other means to provide support for themselves or their families“ und gehen damit auf die mit Leid verbundene Lage der häufig in den Zielländern beheimateten Organspenderinnen ein (Budiani-Saberi und Delmonico 2008, S. 925).

Es fällt also auf, dass Leid vor allem in Texten erwähnt wird, die den Ausdruck Transplantationstourismus erwähnen. In diesen Texten geht es häufig um Patientinnen, die auf der Suche nach einem passenden Organ in ein anderes Land fahren, um dort eines zu kaufen. Tatsächlich wird anhand solcher Beispiele, in denen die in dem Zielland lebenden Spenderinnen häufig aus der Not heraus ein Organ verkaufen, das durch Reisen zu medizinischen Zwecken verursachte Leid besonders deutlich. Das Leid der zu medizinischen Zwecken Reisenden wird dagegen deutlich seltener erwähnt. Um also die Ergebnisse zu dem semantischen Feld Leid differenziert darzustellen, wurden diese weiter in die Untergruppen Leid allgemein, Leid Empfängerin, Leid Spenderin unterschieden. Für die Gruppe Leid allgemein kann das Zitat von Ranjeet Jeevan und Anthony Armstrong als Beispiel vorgestellt werden, die in ihrem Artikel die Ausgangslage vieler Patientinnen beschreiben, die für eine Operation ins Ausland reisen:

\begin{abstract}
„However, such patients are unlikely to see their surgeon or discuss procedures with them prior to travelling out for their surgery. They are also usually required to pay for a 'package' deal prior to travel. Having travelled to a distant location for this purpose, there is significant financial and psychological pressure to proceed with surgery“ (Jeevan und Armstrong 2008, S. 1423).
\end{abstract}

Auf das Leid von Organempfängerinnen geht Tazeen H. Jafar in seinem Artikel explizit ein:

„The present median waiting time on the transplant list in the United States is 13 years and is projected to increase substantially during the next few years. A longer waiting time on dialysis therapy before transplant has been correlated with a poorer outcome. It therefore is recommended that patients with kidney failure undergo transplant as early as possible“ (Jafar 2009, S. 1145).

Die folgende Grafik illustriert zum einen die Häufigkeit des semantischen Felds Leid in Bezug auf die Häufigkeiten der anderen untersuchten semantischen Felder, zum anderen wird das semantische Feld Leid in seine Untergruppen differenziert dargestellt. Zum besseren Verständnis des Verhältnisses der Untergruppen zu den semantischen Feldern Wirtschaft, Ortswechsel und Freizeit werden in der folgenden Abbildung die Untergruppen des semantischen Feldes Leid grafisch hervorgehoben. 


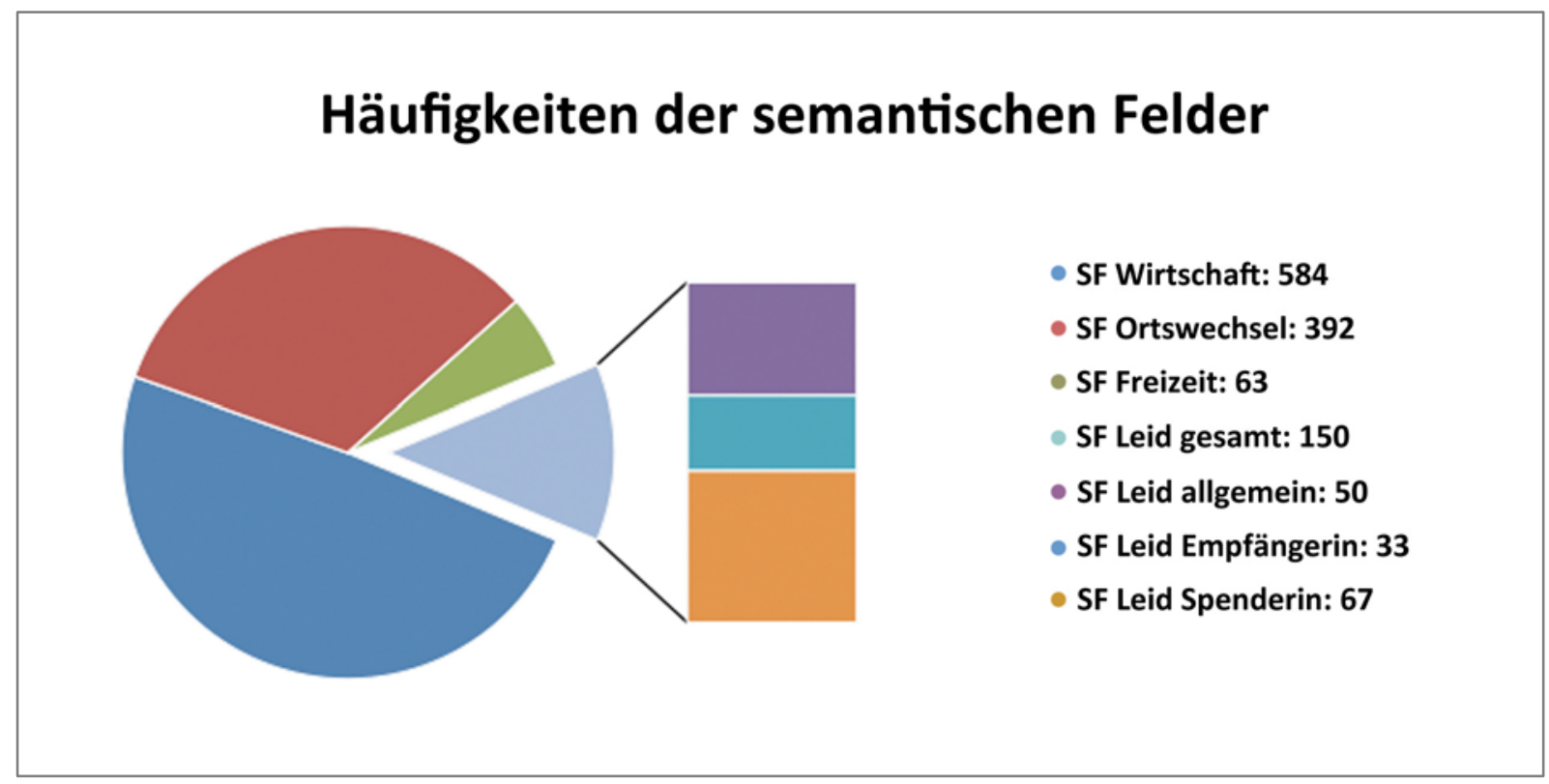

Abb. 10: Häufigkeiten der den semantischen Feldern zugeordneten Zitate im gesamten Textkorpus (absolute Anzahl der Zitate).

An der Grafik lässt sich zum einen ablesen, dass das semantische Feld Leid (hellblaues Tortenstück) insgesamt häufiger verwendet wird als das semantische Feld Freizeit (grünes Tortenstück). Der Balken zeigt die Häufigkeiten der Untergruppen des semantischen Feldes Leid: Dabei kommt es am häufigsten zur Verwendung des semantischen Feld Leid in Bezug auf Organspenderinnen (orangefarbener Teil des Balkens, insgesamt 67 Zitate), gefolgt von Leid allgemein (lilafarbener Teil des Balkens, insgesamt 50 Zitate) und Leid Empfängerin (türkisfarbener Teil des Balkens, insgesamt 33 Zitate).

Die Ergebnisse zeigen, dass die Metapher Medizintourismus besser dazu geeignet ist, das Leid der Betroffenen in den Zielländern zu reflektieren als das Leid der reisenden Patientinnen. Letztere werden als Touristinnen konzeptionalisiert, die typischerweise aus Freizeitgründen und zur Erholung reisen, nicht aber aus einem Leidensdruck. Diese Diskrepanz zwischen der Darstellung des Leidens der Bevölkerung in den Zielländern und der Darstellung des Leidens der reisenden Patientinnen entspricht den Strukturen der Auseinandersetzung mit dem Phänomen „Tourismus" durch die kritische Tourismusforschung. Diese setzt sich mit den Einflüssen von Tourismus auf Zielländer und deren Bevölkerung auseinander.

Eine innovative Funktion der Metapher führt also einerseits im Sinne der kritischen Tourismusforschung dazu, die Auswirkungen des Phänomens auf die Bevölkerung der Zielländer besser wahrzunehmen, sie vermindert aber andererseits die Sensibilität für das Leid der reisenden Personen, da diese vorwiegend als Touristinnen und nicht als Patientinnen konzeptionalisiert 
werden. Am Beispiel des Leidaspektes lassen sich daher sowohl die innovative als auch die konstitutive Funktion nachvollziehen: Während die negativen Auswirkungen auf die Bevölkerung der Zielländer durch die innovative Funktion besser erkannt werden, verringert die konstitutive Funktion die Wahrnehmung des Leidens der reisenden Patientinnen. An diesem Beispiel wird deutlich, dass jede erfolgreiche innovative Übertragung der Semantik eines Sachverhaltes auf einen anderen auch eine konstitutive Wirkung haben kann.

Bisher konnten die illustrative, die innovative und die konstitutive Leistung der Metapher Medizintourismus herausgearbeitet werden. Im folgenden Kapitel wird dargelegt, wie aus diesen Funktionen normative Aussagen folgen. 


\section{EThische ANALYSE DER NORMATIVEN VERWENDUNG DER METAPHER MEDIZINTOURISMUS}

\subsection{NORMATIVE VERWENDUNGSWEISEN}

Die illustrative und die innovative Funktion bedingen entscheidend die epistemische Normativität einer Metapher. Sie lenken das Verständnis und die Konzeptionalisierung des neuen Sachverhaltes über die Strukturen des alten Sachverhaltes. Aus der zunächst zur Illustration herangezogenen Metapher Tourismus kann schnell die Schlussfolgerung gezogen werden, es handele sich bei der transnationalen Medizin um einen bestimmten Tourismuszweig. Diese Vorstellung wird dadurch verstärkt, dass in der Sprache bereits nach demselben Schema zusammengesetzte Wörter verwendet werden, die als unterschiedliche Sektoren von Tourismus (im Sinne von Tourismus als Wirtschaftszweig) verstanden werden, zum Beispiel Abenteuertourismus, Ökotourismus, Wandertourismus oder Städtetourismus.

Eine Metapher macht die Strukturen, die in beiden Sachverhalten, dem Bild gebenden und dem Bild empfangenden, vorhanden sind, für den neuen Sachverhalt erkennbar. Indem sie so zum Verständnis des neuen Sachverhaltes beiträgt, entfaltet sie eine Überzeugungskraft (Kovács 2009). Diese ist jedoch wahrheitsunabhängig und beruht einzig auf der Übereinstimmung der Gemeinplätze. So können durch den Gebrauch einer Metapher implizit Wertungen artikuliert werden, die allein in dem Vergleich von Aspekten des neuen Phänomens mit solchen des bekannten Phänomens begründet sind. Hier liegt das Potential für die moralische Normativität einer Metapher. Denn über den Vergleich werden keineswegs nur sachliche Informationen, sondern auch implizite Wertungen auf den neuen Sachverhalt übertragen. Dies ist für die ethische Auseinandersetzung mit den beschriebenen Sachverhalten von großer Bedeutung.

Da die moralische Normativität nicht immer scharf von der epistemischen Normativität abzugrenzen ist, werden sie in den folgenden Abschnitten gemeinsam diskutiert. Die normativen Aussagen der Metapher Medizintourismus variieren je nach Kontext und Hyponym. Wer sich mit Sterbetourismus auseinandersetzt, kritisiert implizit etwas anderes als wer von Transplantationstourismus spricht. Die moralische Normativität zeigt sich besonders gut, wenn man die Absichten der Senderinnen eines Diskurses genauer untersucht. Mit der Metapher Medizintourismus können ganz unterschiedliche Werturteile zum Ausdruck gebracht werden. Ich möchte vier unterschiedliche Verwendungsweisen, die anhand der Textanalyse zu belegen sind, genauer 
betrachten: die affirmative, die euphemistische, die pejorative und die kritisierende Verwendungsweise.

Im Folgenden wird mit affirmativ eine bejahende, unterstützende Position gemeint. So gibt es Autorinnen, die den Ausdruck ausdrücklich bejahen und ihn als zutreffend für das Phänomen beschreiben. Anhand der euphemistischen Verwendungsweise kann gezeigt werden, inwiefern der Ausdruck Tourismus dazu führt, wichtige Aspekte zu verschleiern. Als Beispiel kann dafür die Zuordnung von illegalem Organhandel zu transplant tourism stehen. Bei der pejorativen Verwendungsweise kommt es über den Gebrauch der Metapher zu einer impliziten negativen Wertung des Sachverhaltes, ohne dass dies näher erläutert oder begründet werden würde. Die kritisierende Verwendungsweise unterscheidet sich von der pejorativen Verwendungsweise insofern, als die Autorinnen differenzierter über das Phänomen berichten und explizit Stellung dazu beziehen. Die Metapher dient dabei der Illustration ihrer Position. In den folgenden Abschnitten werden diese unterschiedlichen Verwendungsweisen anhand von Beispielen eingehend diskutiert.

\subsubsection{AFFIRMATIVE VERWENDUNGSWEISE}

Bei der affirmativen Verwendungsweise kann unterschieden werden, ob der Ausdruck selbst positiv bewertet wird, ob er in der Absicht verwendet wird, das Phänomen in bejahender Weise darzustellen, oder ob die Autorinnen Strukturen beschreiben, die den Ausdruck Tourismus bestätigen. So werden in den Texten zum Beispiel Reiseveranstalterinnen beschrieben, die ihr traditionelles touristisches Angebot um die Organisation von Reisen zu medizinischen Zwecken erweitern. Diese stellen selbst eine Bestätigung (Affirmation) des Ausdrucks dar, denn sie verorten sich offensichtlich in dem neuen Phänomen in einer Weise, als handele es sich tatsächlich um einen Tourismuszweig. Darstellungen solcher affirmativer Strukturen müssen nicht per se mit einer bejahenden Position verbunden sein. Sie können deskriptiv, positiv oder negativ wertend sein. Berichte über diese Strukturen zeigen indes die affirmativen Verwendungsmöglichkeiten auf - in diesem Falle zwar nicht durch die Autorinnen selbst, aber durch bestimmte Akteurinnen im Phänomen. Da sich anhand solcher Affirmationen die Normativität der Metapher gut darstellen lässt, sollen die unterschiedlichen affirmativen Verwendungsweisen gemeinsam in diesem Kapitel anhand konkreter Beispiele erörtert werden.

Kristen Smith spricht sich zum Beispiel ausdrücklich für die Verwendung des Ausdrucks medical tourism aus, damit liegt eine Bejahung des Ausdrucks selbst vor. Sie begründet ihre Affirmation damit, dass ihrer Meinung nach allein durch die Zusammensetzung der eigentlich voneinander 
unabhängigen Ausdrücke medical und tourism die ethischen Fragestellungen hervorgehoben werden:

Similarly, an increasing number of academics have disputed the use of the word 'tourism' due to what is regarded as its ill-fitting, ,suggestion of leisure and frivolity' and lack of neutrality. However, it is precisely this reason that makes it entirely appropriate, as the coupling of the two terms directly highlights the bioethical concerns inherent in the industry. (Smith 2012, S. 2-3)

Die Ergebnisse der vorliegenden Arbeit weisen hingegen nicht darauf hin, dass der Ausdruck gut dazu geeignet ist, die ethischen Bedenken zu unterstreichen und zu thematisieren. Die Untersuchung von Artikeln aus der ethischen Debatte konnte hingegen zeigen, inwiefern Texte, die den Ausdruck medical tourism verwenden, von der Semantik von Tourismus geprägt sind. Bei der positiven Bewertung des Ausdrucks medical tourism durch Kristen Smith handelt es sich also um eine affirmative Position dem Ausdruck gegenüber, die losgelöst ist von ihrer Haltung zu dem Phänomen selbst.

Wenn hingegen Patientinnen, die ursprünglich für eine medizinische Behandlung gereist sind, Sightseeing-Aktivitäten an ihren Krankenhausaufenthalt anschließen, stellt dies eine Affirmation durch die Akteurinnen im Phänomen dar. So berichtet Pafford zum Beispiel über die Patientin Greta Smith, die für eine Bypass-Operation aus den USA nach Indien reiste: "In the morning she undergoes surgery and, after a few days, continues her recovery at a four-star hotel next to the hospital - complete with assisted tours to nearby sights" (Pafford 2009, S. 810). Ob dies sachlich richtig oder gar aus ärztlicher Sicht vernünftig ist, soll hier nicht weiter diskutiert werden. So, wie die Autorin die Situation beschreibt, verhält die Patientin sich aber, wie man es von einer Touristin kennt: Sie wohnt in einem Hotel und schaut sich Sehenswürdigkeiten an. In diesem Sinne verhält Greta Smith sich affirmativ zu traditionell touristischem Handeln. Sind Strukturen wie Sightseeing im Anschluss an eine medizinische Intervention einmal schlüssig im Sinne von Tourismus interpretiert, wird dadurch der Ausdruck im wörtlichen Sinne bestätigt und als zutreffend für den Sachverhalt anerkannt.

Vermittlungsagenturen stellen ebenfalls eine affirmative Analogie zwischen Tourismus und Reisen zu medizinischen Zwecken dar. Sie können dadurch leicht dem Phänomen Tourismus zugeordnet werden und bestätigen den neuen, als Medizintourismus bezeichneten Sachverhalt als Tourismuszweig. Darüber hinaus sind sie durch die Semantik von Tourismus als Wirtschaftszweig legitimiert, diese Reisen zu vermitteln, zu bewerben und daran zu verdienen. Kristen Smith beschreibt in ihrem Artikel „The problematization of medical tourism: a critique of neoliberalism“ diese affirmative Selbstverortung der Agenturen folgendermaßen: „Although individuals certainly travel for purposes outside the traditional realms of commonly understood 
notions of tourism, the industry itself, in both its promotion and presentation, positions itself firmly within this sphere" (Smith 2012, S. 3).

Wenn Agenturen oder Kliniken selbst den Ausdruck medical tourism verwenden, stellen sie nicht nur selbst eine Affirmation dar, sie verwenden den Ausdruck beziehungsweise das Vokabular der zugehörigen semantischen Felder auch in affirmativer Weise. So beschreibt der Bioethiker Leigh Turner, wie Vermittlungsagenturen Vokabular aus dem semantischen Feld Wirtschaft dazu nutzen, Patientinnen als Konsumentinnen anzusprechen:

Dental tourism companies promote cross-border care using the rhetoric of consumer choice. This language suits circumstances where patients can choose between receiving care from local dentists and travelling abroad for treatment. [...] Where patients cannot afford access to local dentists, constraints rather than choices shape decisions to travel (Turner 2009, S. 118).

Die Nucci Medical Clinic in Florida wirbt zum Beispiel mit folgendem Bild, um Patientinnen aus dem Ausland auf eine Behandlung an ihrer Klinik aufmerksam zu machen:

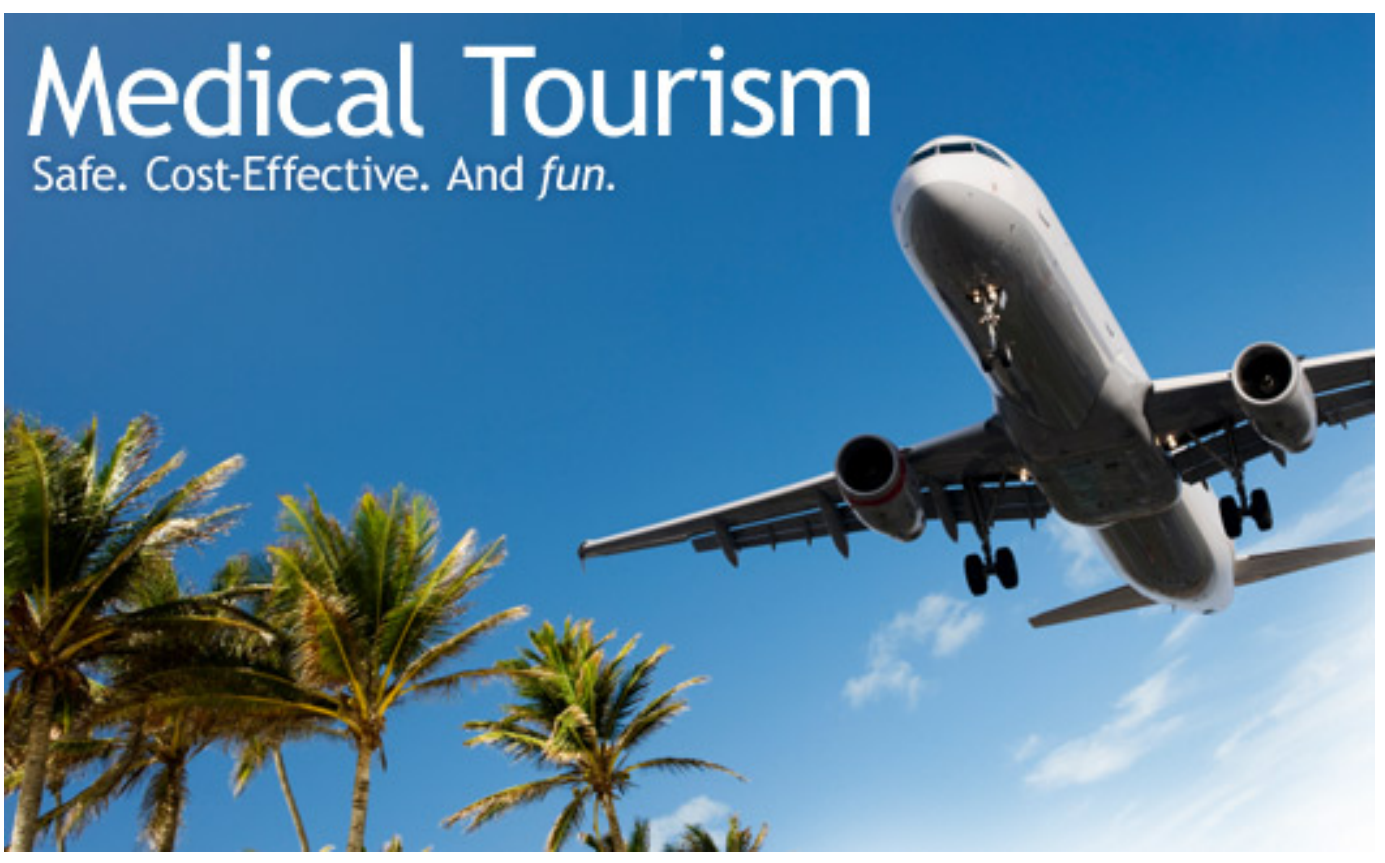

Abb. 11: Ausschnitt aus dem Internetauftritt der Nucci Medical Clinic in Florida (Website Nucci Medical Clinic)

In diesem Bild wird der Ausdruck medical tourism mit blauem Himmel, Sonne und Palmen in Zusammenhang gebracht. Genau so könnte die Werbung für einen Urlaub in der Karibik aussehen. Es werden visuell keinerlei Hinweise auf eine medizinische Behandlung, Krankheit oder Leiden gegeben. Die Klinik nutzt also die Semantik Freizeit des Ausdrucks Tourismus, um potentielle, angenehme Seiten einer Reise zu medizinischen Zwecken in den Vordergrund zu stellen und so Kundinnen zu werben. Weitere Bespiele zur affirmativen Verwendungsweise durch Akteurinnen finden sich bei dem rumänischen Vermittlungsunternehmen Qdays, das ihren Kun- 
dinnen „true holiday“ verspricht, oder dem Vermittlungsunternehmen MedRetreat, das mit dem Slogan,... where smart medicine and exotic travel come together" wirbt (Website Qdays; Website MedRetreat). Darüber hinaus sind die Websites dieser Unternehmen mit Bildern von Stränden, Palmen oder Hotels mit Pool illustriert. Die Agenturen heben mit Hilfe der Metapher bewusst angenehme Seiten des Reisens hervor, um den Patientinnen eine Reise aufgrund einer medizinischen Behandlung attraktiver erscheinen zu lassen.

Auch im Zusammenhang mit transplant tourism kommt es zu einer affirmativen Verwendungsweise durch Akteurinnen selbst. Turner weist darauf hin, dass das indische Ministerium für Tourismus auf seiner Website Incredible India Nierentransplantationen bewirbt: „In India, while some government officials are trying to eliminate the country's underground economy in the sale of human organs, the Ministry of Tourism promotes the sale of organ transplants and the country's organ transplant facilities actively solicit international patients“ (Turner 2008, S. 393). Die Tatsache, dass das Ministerium für Tourismus eine Rubrik Medical Tourism auf seiner Website führt, zeigt die affirmative Selbstverortung des Ministeriums und führt wiederum zu einer Bestätigung des ursprünglich als Metapher gemeinten Ausdrucks Tourismus im Zusammenhang mit medizinischen Behandlungen (Website Ministry of Tourism India). An diesem Beispiel wird aber auch deutlich, dass Affirmation durch Handlungen bestimmter Akteurinnen und eine affirmative Verwendungsweise des Ausdrucks nicht immer scharf voneinander zu trennen sind. Lakoff und Johnson stellen fest, dass das durch die Metapher wahrgenommene Bild „den Status einer Wahrheit annehmen“ kann. Deshalb könne „die Metapher einen Rückkoppelungseffekt haben in dem Sinne, dass wir uns bei unseren zukünftigen Handlungen von der Metapher leiten lassen“ (Lakoff und Johnson 2004, S. 164). So fühlt sich also eine Reiseveranstalterin von dem Ausdruck Tourismus angesprochen und nimmt daraufhin „medizintouristische Reisen“ in ihr Angebot mit auf.

Die werbenden Agenturen und Behörden bewegen sich zwischen der epistemischen und der moralischen Normativität der Metapher Medizintourismus. Sie fühlen sich durch den Ausdruck Tourismus angesprochen und verorten sich analog zu ihrer Position im „Tourismus“ in dem neuen, als Medizintourismus bezeichneten Phänomen. Sie greifen den Tourismus-Begriff auf und interpretieren ihre Angebote in diesem Sinne. Dadurch leiten sie das Verständnis der Adressatin, in diesem Falle der Patientin, von dem Phänomen auf Aspekte, von denen sie profitieren, zum Beispiel den Verkauf von Sightseeing-Aktivitäten am Zielort. Damit schaffen sie zugleich eine Möglichkeit, wertende Konnotationen auf das Gesamtphänomen zu übertragen. Diese Konnotationen variieren je nach Standpunkt. Die Konnotation „Freizeittourismus“ ist für die Vermittlungsagenturen förderlich, da sie in erster Linie ein Produkt vermarkten wollen und die Patien- 
tinnen mit der Vorstellung eines schönen Urlaubs locken. Die Realität der Krankheit, verbunden mit Leiden und Hilfsbedürftigkeit, wird marginalisiert.

Zusammenfassend konnte gezeigt werden, dass eine Affirmation des Ausdrucks Tourismus auf mehreren Ebenen stattfindet. Manche Autorinnen sprechen sich explizit für die Beibehaltung des Ausdrucks aus, andere Akteurinnen bestätigen den Ausdruck, indem sie sich analog zu traditionell touristischem Handeln verhalten. Dies wurde sowohl anhand konkreter Werbungsbeispiele einiger Unternehmen als auch anhand von Berichten über reisende Individuen illustriert. Hieran konnte gezeigt werden, dass es Akteurinnen wie Vermittlungsagenturen durch den affirmativen Einsatz der Metapher gelingt, die Wirkungen des Ausdrucks gezielt für sich zu nutzen.

\subsubsection{EUPHEMISTISCHE VERWENDUNGSWEISE}

Neben der affirmativen Verwendungsweise der Metapher Medizintourismus fanden sich in dem untersuchten Textmaterial auch Beispiele für eine euphemistische Verwendungsweise. Euphemistisch meint in diesem Zusammenhang, dass tatsächliche Zusammenhänge beschönigend dargestellt werden. So werden zum Beispiel illegale Aspekte im Zusammenhang mit internationalen Transplantationen mit dem Ausdruck transplant tourism sprachlich verschleiert, da der Ausdruck tourism diese illegalen Aspekte nicht berücksichtigt. Die euphemistische Verwendungsweise zeigte sich vor allem bei der Verwendung der Ausdrücke transplant tourism und reproductive tourism.

So wird zum Beispiel in den Texten, die den Ausdruck transplant tourism verwenden, die Frage der Legalisierung einer Kommerzialisierung von Organtransplantationen mit Vokabular aus dem semantischen Feld Wirtschaft diskutiert. Über die Verwendung von Vokabular aus dem semantischen Feld Wirtschaft in Verbindung mit dem Ausdruck Tourismus entsteht bei den Adressatinnen indirekt der Eindruck, es handele sich um einen legalen Wirtschaftszweig. Schließlich entspricht dies einem der drei semantischen Hauptaspekte des Ausdrucks Tourismus. Tatsächlich wird das semantische Feld Wirtschaft in Texten, in denen der Ausdruck transplant tourism verwendet wird, im Durchschnitt in 16 Zitaten bedient. Gleichzeitig wird aber in den Explikationen von transplant tourism keine inhaltliche Zuordnung als Wirtschaftszweig vorgenommen. Im Gegenteil zu einer solchen Zuordnung wird transplant tourism häufig als eine Form von illegalem Organhandel definiert. Es handelt sich also bei dem Ausdruck transplant tourism in dieser Hinsicht um einen Euphemismus.

Diese Wirkung wird von den Autorinnen unterschätzt. Einige von ihnen versuchen zwar, die Illegalität von "transplant tourism" ausdrücklich hervorzuheben. Da aber erstens nicht alle Autorinnen den Ausdruck präzisieren und zweitens eine Vielzahl an unterschiedlichen Explikatio- 
nen verwendet wird, kann diese Umdefinierung entgegen der ursprünglichen Semantik die Konnotation von Tourismus als legalem Wirtschaftszweig nicht verhindern. Verfechterinnen der Legalisierung der kommerzialisierten Organtransplantation kommt diese euphemistische Verwendungsweise entgegen, wird doch allein über die Sprache bewirkt, dass Zustände bereits als legal wahrgenommen werden, die es erst zu legalisieren gilt. Problematisch ist daran, dass dabei eine Diskussion aller Argumente auf rein sprachlichem Wege ausgehöhlt wird.

Auch Reproduktionstourismus wird von unterschiedlichen Akteurinnen euphemistisch verwendet. Zum einen geschieht dies durch die Anbieterinnen, seien es Kliniken oder Vermittlungsagenturen, die Patientinnen werben wollen und dies über die Suggestion eines Urlaubes tun, während sie die Tatsache, dass es sich um eine medizinische Behandlung handelt, unterschlagen. Der Übergang von einer affirmativen zu einer euphemistischen Verwendungsweise ist dabei fließend.

Zum anderen nutzen Patientinnen selbst die Metapher, um der Missbilligung durch ihr Umfeld zu entgehen, selbst wenn sich ihre eigenen Erfahrungen damit nicht decken. Patientinnen, die für reproduktionsmedizinische Behandlungen ins Ausland reisen, erleben häufig gesellschaftliche Missbilligung. Indem sie die Fassade eines Erholungsurlaubes aufbauen, verstärken sie nach außen hin die Tourismusillusion. Da dieses Bild nicht ihrer erlebten Wirklichkeit entspricht, liegt hier ebenfalls eine euphemistische Verwendungsweise vor. Diesen Effekt beschreiben Naraindas und Bastos für muslimische Patientinnen aus den Ländern des Nahen Ostens: „Disallowed by Sunni law to have donor eggs, the couple goes in search of Shia gametes. Since any kind of surrogacy is frowned upon, their travel is conducted under the guise of a holiday. Here the holiday functions as a trope for a painful and traumatic quest" (Naraindas und Bastos 2011, S. 4). Da das sunnitische Recht den Patientinnen eine Eizellspende verbietet, reisen sie dafür ins Ausland. Die Urlaubsreise muss hier als Deckmantel für die für das Kinderwunschpaar häufig schmerzhafte Suche nach der Erfüllung seines Kinderwunsches herhalten.

Konnotationen des Ausdrucks Tourismus können aber auch verwendet werden, um Kritik an dem Phänomen zu äußern. Hierbei kann eine lediglich pejorative von einer explizit kritisierenden Verwendungsweise unterschieden werden.

\subsubsection{PEJORATIVE VERWENDUNGSWEISE}

In der Debatte um die Angemessenheit des Ausdrucks Reproduktionstourismus (vgl. Kapitel 1.6) wird als Argument gegen die Verwendung angeführt, dass der Ausdruck häufig dazu genutzt wird, in polemischer Weise die Situation der Betroffenen zu beschreiben. So wiesen bereits Inhorn und Patrizio, die eine Studie mit aus reproduktionsmedizinischen Gründen reisenden Pati- 
entinnen und Patienten durchgeführt haben, auf das normative Potential des Ausdrucks reproductive tourism hin: „Because reproductive tourism implies fun, leisure, and holidays under the sun, it is a term that is cavalier and insensitive" (Inhorn und Patrizio 2009, S. 905). Im Folgenden soll die pejorative Verwendungsweise anhand von Ergebnissen aus meiner Textanalyse verdeutlicht werden.

In ihrem Artikel „So weit gehen für ein Kind“ geht die Sozialanthropologin Eva-Maria Knoll auf Vermittlungsagenturen ein, die „gezielt die Verknüpfung einer IVF mit entspannter Urlaubsatmosphäre im luxuriösen Zimmer“ vermarkten (Knoll 2008, S. 67). Knoll verwendet den Ausdruck Reproduktionstourismus im Zusammenhang mit Agenturen, die den Ausdruck affirmativ für sich nutzen, impliziert dabei aber zum Beispiel mit inhaltlichen Verbindungen wie „reproduktives Globetrotten - Gesetzesumgehung und mehr" eine Illegalität, die nicht gegeben ist. Denn nicht jeder „Reproduktionstourismus“ spielt sich in den „Randzonen der Legalität“ (Knoll 2008, S. 70-71) ab. So kommt es zu einer polemischen Verwendungsweise der Metapher, mit deren Hilfe die Autorin implizit Kritik am vermeintlich illegalen Verhalten der Betroffenen äuBert.

Mit Ausdrücken wie „IVF-Holiday“, „Reproduktives Globetrotten“ oder „Reprotouristen“ entwickelt Knoll die ursprüngliche Tourismus-Metapher sogar noch weiter zu Ausdrücken, die den Wirtschaftsaspekt nur noch unterschwellig mit sich führen und den Freizeit- und Erholungsaspekt hervorheben (Knoll 2008, S. 67, S. 70, S. 77). Ebenso ist dies bei Untis sprachlicher Neuschöpfung „procreation vacation“ der Fall (Unti 2009, S. 19).

Holiday, globetrotten und vacation sind Ausdrücke, die zur Semantik von Tourismus gehören. Dennoch sind sie nicht gleichbedeutend mit Tourismus. IVF-Holiday wird analog zu Reproduktionstourismus als Determinativkompositum gebildet. Dabei wird die schon vorher verkürzte Spezifizierung (Reproduktion statt Reproduktionsmedizin) noch stärker verkürzt und verfremdet (IVF). Der ursprüngliche Reisegrund, die reproduktionsmedizinische Behandlung, wird auf eine Abkürzung reduziert. Zudem erfolgt über den Ausdruck Holiday eine Interpretation des Ausdrucks Tourismus in Richtung des Freizeitaspektes. So wird Freiwilligkeit und positives Erleben der Situation durch die Betroffenen unterstellt, die in den wenigen Studien, in denen Betroffene tatsächlich zu ihrer Situation befragt wurden, nicht zu erkennen sind. So gaben betroffene Patientinnen in der Studie von Inhorn und Patrizio an, dass sie eine Behandlung im Heimatland vorgezogen hätten: „In virtually every case, infertile couples describe their preferences not to travel if only legal, trustworthy, and economical services were made available closer to home“ (Inhorn und Patrizio 2009, S. 905). 
Obwohl Anthropologin, vernachlässigt Knoll, dass der Ausdruck Reproduktionstourismus die Wirklichkeit der Patientinnen nicht widerspiegelt. Die Metapher wird von der Autorin kreativ weiterentwickelt, um bestimmte Konnotationen zu betonen. Der Blickwinkel der Rezipientin wird auf Kosten der Betroffenen auf einen kleinen Teil der Realität der reisenden Patientinnen und Patienten gelenkt. Patientinnen und Patienten, die aus reproduktionsmedizinischen Gründen reisen, fühlen sich hingegen durch den Ausdruck diskriminiert, da er ihre Verzweiflung nicht berücksichtige (Inhorn und Shrivastav 2010). Knoll marginalisiert den Leidensdruck der Betroffenen und kritisiert implizit das Verhalten, für reproduktionsmedizinische Behandlungen den Wohnort zu verlassen.

Der Rechtswissenschaftler Richard Storrow vergleicht Reproduktionstourismus gar mit Sextourismus: „I conclude that, on balance, fertility tourism raises many of the same concerns raised by sex tourism“ (Storrow 2005, S. 299). Durch diesen Vergleich werden implizit moralische Wertungen des bekannteren Sachverhaltes, in diesem Fall Sextourismus, auf den unbekannteren, in diesem Fall Reproduktionstourismus, übertragen. Der Vergleich scheint zunächst allein aufgrund der Ähnlichkeit der Morphologie der Ausdrücke plausibel. Beide Wörter sind nach den Regeln der Bildung von Determinativkomposita zusammengesetzte Ausdrücke und haben denselben semantischen Kopf, Tourismus. Morphologisch gesehen sind sich Reproduktionstourismus und Sextourismus genauso nah wie Reproduktionstourismus und Medizintourismus. Hier wird die Problematik der Verkürzung des Hyponyms Reproduktionstourismus deutlich: als Hyponym von Medizintourismus müsste es folgerichtig Reproduktionsmedizintourismus heißen. Reproduktionsmedizin ist rein morphologisch deutlich von Sex abzugrenzen. Die Abgrenzung von Reproduktion zu Sex ist hingegen wesentlich weniger deutlich. Über den Vergleich von Reproduktionstourismus zu Sextourismus gelingen weitreichende moralische Bewertungen des Sachverhaltes, die nicht in dem Phänomen begründet liegen. Prostitution gilt vielen generell als moralisch verwerflich, umso mehr, wenn eine Person ins Ausland fährt und dort Geld für Sex bezahlt, den sie im Heimatland nicht haben würde/könnte. Im Zusammenhang von Sextourismus wird zudem selten zwischen gegebenenfalls legaler Prostitution und illegaler Kinderprostitution unterschieden. Letztere ist zumeist in den Zielländern ebenso verboten wie im Heimatland. Über die Morphologie der beiden inhaltlich voneinander nicht im Zusammenhang stehenden Metaphern wird also eine implizite Wertung von aus medizinischen Gründen Reisenden als moralisch schlecht begünstigt.

Berücksichtigt man die Patientinnensicht, muss der Ausdruck Reproduktionstourismus als pejorativ und polemisch verstanden werden. Es liegen zwar keine Untersuchungen mit betroffenen Patientinnen aus anderen medizinischen Bereichen zu dieser Fragestellung vor. Da jedoch auch 
andere Patientinnen krank sind und häufig nur reisen, weil ihnen die gesuchte Behandlung im Heimatland nicht zur Verfügung steht, liegt die Annahme nahe, dass Patientinnen aus anderen medizinischen Fachbereichen sich durch die Tourismus-Metapher ebenso diskriminiert fühlen können.

Auch hier zeigt sich wieder die Möglichkeit, über die Anwendung ein- und derselben Metapher unterschiedlichste moralische Wertungen auszudrücken. Dies geschieht ebenso bei der kritisierenden Verwendungsweise.

\subsubsection{KRITISIERENDE VERWENDUNGSWEISE}

Während bei der pejorativen Verwendungsweise eine implizite negative Bewertung bestimmter Aspekte im Vordergrund steht, wird die Metapher Medizintourismus von einigen Autorinnen in der Absicht verwendet, ihre explizit geäußerte Kritik an bestimmten Aspekten des Phänomens zu illustrieren. Dies lässt sich besonders bei der Verwendung von transplant tourism zeigen. In diesen Texten wird die Tourismusmetapher häufig dazu genutzt, um die wirtschaftlichen Strukturen hervorzuheben und somit auf eine als problematisch bewertete Kommerzialisierung des Systems hinzuweisen.

So verwenden Budiani-Saberi und Delmonico in ihren Texten den Ausdruck Tourismus in Verbindung mit Vokabular aus dem semantischen Feld Wirtschaft, um illegale kommerzielle Strukturen im Zusammenhang mit transnationaler Transplantationsmedizin explizit zu kritisieren. Sie schreiben aus der Sicht eines Transplantationsmediziners (Delmonico) und einer MedizinAnthropologin (Budiani-Saberi), denen vermutlich bewusst ist, dass das semantische Feld der Transplantationsmedizin von Vokabular geprägt ist, das Altruismus und nicht monetäre Interessen ausdrückt. So handelt es sich bei den Beteiligten einer Organspende um Spenderinnen und Empfängerinnen. Dieses Vokabular trägt eine hohe moralische Normativität, denn die altruistische Spende wird, nicht nur im Bereich der Transplantationsmedizin, in unserer Gesellschaft sehr positiv bewertet. Die Kritikerinnen nutzen nun Vokabular aus ökonomischen Zusammenhängen, um aufzuzeigen, dass es sich in einigen Bereichen der Transplantationsmedizin entgegen weit verbreiteten Vorstellungen nicht um von Altruismus und dem Wohl der Patientinnen, sondern um von monetären Interessen geleitete Systeme handelt.

So wechseln zum Beispiel Budiani-Saberi und Delmonico zwischen Ausdrücken aus dem semantischen Feld Wirtschaft (zum Beispiel „kidney vendor“) und solchen aus dem Bereich des Altruismus (zum Beispiel „donation“) hin und her. Besonders auffällig sind Komposita aus sich eigentlich widersprechenden Wörtern aus beiden Feldern, zum Beispiel „commercial donation“ beziehungsweise „commercial living donor" (CLD). Im Folgenden wird eine Passage aus dem Aufsatz 
„Organ trafficking and transplant tourism: a commentary on the global realities" zitiert, in der Budiani-Saberi und Delmonico die Konsequenzen von Organhandel für Betroffene beschreiben, die eine Niere zur Transplantation zur Verfügung gestellt haben, und in der sie die Umstände und ihre Einbettung in ökonomische Prozesse auf das Schärfste kritisieren. In diesem Abschnitt verwenden die Autorinnen auffällig viel Vokabular aus dem semantischen Feld Wirtschaft:

\section{The Consequence to the Vendors}

What then of this emerging worldwide population of live kidney vendors? In Pakistan, the SIUT [Sindh Institute of Urology and Transplantation] group has carefully detailed a sample cohort of $(\mathrm{n}=239)$ vendors in a follow- up-the outcome all very troubling (12). The majority of these CLDs (93\%) who sold a kidney to repay a debt and (85\%) reported no economic improvement in their lives, as they were either still in debt or were unable to achieve their objective in selling the kidney. The disturbing report by the SIUT group becomes not only an accounting of the Pakistani experience but an indictment of the international transplant community because it overlooks the plight of the donor whose interests are just as valid as the recipients. Egypt is one of the few countries that prohibits organ donation from deceased donors. In the absence of an entity to govern allocation or standards for transplants, the market has become the distribution mechanism. Egypt is also one of the countries in which COFS [Coalition for Organ-Failure Solutions] has conducted extensive field research and long-term outreach service programs for victims of the organ trade. In-depth longitudinal interviews conducted by Budiani reveal that $78 \%$ of the CLDs $(n=50)$ reported a deterioration in their health condition. This is likely a result of factors such as insufficient donor medical screening for a donation, preexisting compromised health conditions of CLD groups and that the majority of employed CLDs reported working in labor-intensive jobs. A kidney sale does not solve the most frequently given reason for being a CLD, 81\% spent the money within 5 months of nephrectomy, mostly to pay off financial debts rather than investing in quality of life enhancements. CLDs are not eager to reveal their identity; $91 \%$ expressed social isolation about their donation and $85 \%$ were unwilling to be known publicly as an organ vendor. Ninety-four percent regretted their donation (13). The studies in Pakistan and Egypt are consistent with findings in India (14), Iran (15) and the Philippines (16) that revealed deterioration in the health condition of the CLDs. A long-term financial disadvantage is evident following nephrectomy from a compromised ability to generate a prior income level. The common experience also entails a social rejection and regret about their commercial donation (Budiani-Saberi und Delmonico 2008, S. 927-928) (Hervorhebungen durch M.M.).

Anhand dieses ausführlichen Zitates zeigt sich, wie das Vokabular aus dem semantischen Feld Wirtschaft dazu genutzt werden kann, um kommerzielle Strukturen im Zusammenhang mit internationaler Organtransplantation zu kritisieren.

Auch bei Turner findet sich die kritisierende Verwendungsweise, speziell im Hinblick auf reisende Patientinnen im Bereich der Transplantationsmedizin. Während er in seinem Text „,Medical tourism' initiatives should exclude commercial organ transplantation“ die transnationale Medizin zunächst als sich ausbreitende "medical tourism industry“ beschreibt, nutzt er später Vokabular aus dem wirtschaftlichen Bereich, um den Organhandel zu verurteilen: „Given the many dangers associated with selling purchased organs to medical tourists, organ transplant facilities and transplant procedures should be excluded from medical tourism initiatives" (Turner 2008, S. 393). Turner erkennt die Gefahren, die dabei von den vermittelnden Unternehmen ausgehen und kritisiert deren Rolle in dem als tourism bezeichneten Phänomen: „Medical tourism campaigns exacerbate this problem by marketing ,all-inclusive' organ transplant packages 
to international patients. These operations include kidneys purchased from poor individuals rather than obtained from living donors or deceased persons" (Turner 2008, S. 391). Die Funktion des Gebrauchs von Vokabular aus dem semantischen Feld Wirtschaft reicht in seinem Text von rein beschreibend bis hin zu kritisierend.

Autorinnen wie Turner unterschätzen in ihrer kritisierenden Verwendungsweise die konstitutive Funktion der Metapher. Über die Konnotation von Tourismus als Wirtschaftszweig bewirkt der Ausdruck eine Festschreibung des Phänomens als legalen Wirtschaftszweig. Im Bereich der Transplantationsmedizin ist die kritisierende Verwendungsweise von der Adressatin leichter als solche zu verstehen, da die traditionell vorherrschende Meinung im Bereich der Transplantationsmedizin besagt, dass die Verbindung von Organtransplantation und wirtschaftlichen Interessen moralisch schlecht sei. Je stärker jedoch im Zusammenhang mit anderen Fachbereichen die unterschiedlichen Hyponyme absichtlich mit der Bedeutung „legaler Wirtschaftszweig“ konnotiert werden, umso deutlicher wird sich diese Bedeutung voraussichtlich auch auf den Ausdruck transplant tourism übertragen.

Die Übertragung von Konnotationen erfolgt allerdings immer in beide Richtungen. So zeigen die angeführten Beispiele, dass der Ausdruck transplant tourism entgegen der Semantik von Tourismus als legalem Wirtschaftszweig mit illegalen Zusammenhängen konnotiert wird. Dadurch kommt es zu Umkehrschlüssen im Zusammenhang mit den anderen Hyponymen wie Reproduktionstourismus oder dem Hyperonym Medizintourismus. In diesem Fall liegt kein Euphemismus mehr vor, dies hat im Gegenteil eine Kriminalisierung von legal Handelnden zur Folge. Eine solche Kriminalisierung findet sich bereits in Texten, die andere Hyponyme aufweisen. So schreibt zum Beispiel Knoll:

\footnotetext{
Andererseits stellt Reproduktionstourismus als Phänomen, in dem die Patienten die Dinge einfach selbst in die Hand nehmen, eine gewisse Bedrohung des Einflusses der nationalen Rechtsstaatlichkeit dar, handelt es sich doch mehr oder weniger um ein informelles ökonomisches Segment, das in den Randzonen der Legalität operiert, wenn hierzulande per Gesetz verbotene Behandlungsformen wenige Kilometer weiter, jenseits der Landesgrenzen durchgeführt werden (Knoll 2008, S. 71).
}

Weiter kritisiert sie, „dass Reproduktionstourismus zuallererst eine legislative Umgehungsstrategie sei“ und es sich um die „rechtliche Umgehung“ und Überwindung „nicht nur juristischer Grenzen und Barrieren“ gehe (Knoll 2008, S. 71). Von diesen Formulierungen ist es nicht mehr weit zu der Annahme, aus reproduktionsmedizinischen Gründen reisende Patientinnen würden stets illegal handeln. Dies ist jedoch meistens nicht der Fall. Jeder Person steht es frei, zu einem anderen Ort zu reisen und dort erlaubte medizinische Prozeduren in Anspruch zu nehmen. Dies gilt lediglich nicht für Organhandel, da hier das Extraterritorialitätsprinzip gilt. 
Bei Knolls Kritik, die sich in einer pejorativen Verwendungsweise äußert, geht es um eine Verurteilung dessen, was die Patientinnen tun. Andere Autorinnen kritisieren dagegen die ökonomischen Strukturen und den Profit, der mit der Not der reisenden Patientinnen gemacht wird. So schreibt zum Beispiel Naomi Pfeffer: „Fertility tourism' is used throughout this paper in order to emphasize that clinics in destination countries are motivated by profit and furthermore are part of the multibillion dollar medical tourism industry“ (Pfeffer 2011, S. 638).

Da die kritisierende Verwendungsweise von der pejorativen nicht immer scharf zu trennen ist, wird die kritisierende Verwendungsweise zum Teil selbst Gegenstand von Kritik. So bemängelt Evans die unterschwellig emotionale Argumentation:

\footnotetext{
First, language and concepts are critical to our understanding. Unfortunately, based on negative associations, the concept of transplant tourism has become emotionally charged. For example, ,organ brokers', ,agents' and ,organ trafficking' are frequently mentioned in the same breath as transplant tourism, even though it has long been an established practice. Such language narrows the debate, and biases the process of argumentation (Evans 2008, S. 1091).
}

Evans beklagt die kritisierende Anwendung von Vokabular aus dem semantischen Feld Wirtschaft, ohne den Ausdruck tourism insgesamt in Frage zu stellen. Er verkennt dabei, dass der Aspekt Wirtschaft Teil der Semantik des Ausdrucks Tourismus ist. Über die innovative Funktion lädt dieser gerade dazu ein, entsprechendes Vokabular auf Strukturen in dem Phänomen zu übertragen. Die angeführten Beispiele verdeutlichen die vielfältigen und unübersehbaren Wirkungsweisen, die sich aufgrund der Wechselwirkungen von unterschiedlichen Konnotationen in einem Metaphernsystem entwickeln.

Zusammenfassend kann man wie bei den unterschiedlichen Leistungen der Metapher auch bei den Verwendungsweisen moralische wie epistemische Normativität feststellen. Die epistemische Normativität zeigt sich zum Beispiel daran, dass sich beteiligte Unternehmen affirmativ im Sinne der Metapher verorten. Außenstehende erkennen nun wiederum die Strukturen, die durch die Metapher vorgeschlagen werden. Darüber hinaus wirkt die affirmative Verwendungsweise auch moralisch normativ, indem sie eine Gleichsetzung von Reisen zu medizinischen Zwecken und Urlaub vornimmt. Damit wird eine von den Betroffenen als belastend empfundene Reise als angenehme Freizeitbeschäftigung bewertet. Bei der kritisierenden Verwendungsweise verwenden Autorinnen die Metapher, um zuvor explizit geäußerte Kritik zu illustrieren. Dies wird zum Beispiel dadurch versucht, Vokabular aus dem semantischen Feld Wirtschaft in Bereichen anzuwenden, die traditionellerweise nicht in wirtschaftlichen Rahmen gedacht werden, wie im Bereich der Transplantationsmedizin. Allerdings verblasst die so geäußerte Kritik angesichts der Wechselwirkungen mit den anderen Verwendungsweisen. Der pejorativen und euphemistischen Verwendungsweise ist die implizite Äußerung von Wertungen und Meinungen gemein, 
auch hier lässt sich eine moralische Normativität feststellen. So nutzen die Autorinnen die pejorative Verwendungsweise der Metapher, um ihrer Missbilligung unterschwellig Ausdruck zu verleihen und die Lage der Betroffenen einseitig zu beschreiben. Die Metapher soll in diesem Fall eine bestimmte Meinung suggerieren.

Auf diese Weise implizit geäußerte Positionen sind gerade in der wissenschaftlichen Debatte problematisch, sollte es doch vor allem darum gehen, Argumente explizit zu benennen und auszutauschen. Besonders interessant ist die Tatsache, dass dieselbe Metapher für unterschiedlichste Zwecke benutzt wird. Die vielfältigen, sich teilweise widersprechenden Verwendungsweisen zeigen, dass es stark von der Intention der Autorin abhängt, wie die Metapher Medizintourismus zu verstehen ist. Auch dies erschwert eine offene Debatte über die ethischen Argumente für alle Diskursteilnehmenden.

\subsection{AusWirkungen DER METAPHER MEDIZINTOURISMUS AUF UnSER VERSTÄNDNiS VON EINER GLOBALEN GESUNDHEITSVERSORGUNG}

\subsubsection{GESUNDHEITSVERSORGUNG: WIRTSCHAFTSZWEIG ODER SOZIALES SYSTEM?}

Mit der Diskussion über den Ausdruck Medizintourismus untrennbar verbunden ist die Frage danach, welches Verständnis von Gesundheitssystemen wir der Konzeptionalisierung einer globalen Medizin zugrunde legen. Handelt es sich bei Gesundheitssystemen primär um soziale oder primär um ökonomische Systeme? Ist medizinische Versorgung nichts anderes als eine Dienstleistung, so wäre es nur folgerichtig, diese in Wirtschaftsstrukturen wie jener des Tourismus zu denken, ähnlich wie die Gastronomie, die je nach Nachfrage ihr Angebot auf Einheimische und Touristinnen abstimmt. Eine solche Perspektive ließe jedoch außer Acht, dass in vielen Ländern die Gesundheitsversorgung zumindest zum Teil in solidarischen Sozialsystemen organisiert ist, die gerade nicht vorrangig nach den Regeln des freien Marktes funktionieren.

Natürlich geht es auch in solidarisch organisierten Gesundheitssystemen um Wirtschaftlichkeit und ökonomische Prozesse, die als solche benannt werden müssen. In diesen Zusammenhängen ist es passend und angemessen, Vokabular aus dem wirtschaftlichen Bereich zu verwenden. Im Kontext der transnationalen Medizin erweckt der Ausdruck Tourismus jedoch fälschlicherweise den Eindruck eines freien Marktes. Damit geraten solidarische Strukturen der Gesundheitsversorgung aus dem Blick. Die medizinische Versorgung von Patientinnen ist in vielen Gesellschaften kein Produkt, das auf dem freien Markt gehandelt wird, sondern ein Gut, das vorrangig unter Gesichtspunkten der Solidarität reguliert und bedürfnisorientiert verteilt wird. 
Gesundheitsversorgung wird hingegen im Bereich der transnationalen Medizin häufig als vorrangig wirtschaftliches System konzeptionalisiert. So beschreibt zum Beispiel Unti das Phänomen reisender Patientinnen als ein Resultat nahezu unkontrollierbarer Marktkräfte: „An important point for all practitioners to understand is that the entire medical tourism phenomenon is being driven purely by economic market-place forces, and so far its rapid growth has occurred largely outside of the view and control of organized medicine" (Unti 2009, S. 21) (Hervorhebung durch M.M.). Kristen Smith kritisiert in ihrem Aufsatz „The problematization of medical tourism: a critique of neoliberalism" eben diese Konzeptionalisierung des als medical tourism bezeichneten Phänomens als vorwiegend ökonomisches System. Sie appelliert an die Diskursteilnehmenden, auch über neoliberale Denkmuster hinausgehende Argumente wieder stärker in den Blick zu nehmen:

Within this milieu, medical tourism is being promoted as a rational economic development strategy for some developing nations, and a makeshift solution to the escalating waiting lists and exorbitant costs of health care in developed nations. This paper explores the need to problematize medical tourism in order to move beyond one dimensional neoliberal discourses that have, to date dominated the arena. (Smith 2012, S. 1).

Kristen Smith schlägt vor, das Phänomen unter Berücksichtigung eines Menschenrechtes auf medizinische Versorgung zu diskutieren. Da sie aber selbst weiterhin die Tourismus-Metapher verwendet, bleibt ihr Anliegen ambivalent. Die konstitutive Leistung der Tourismus-Metapher fördert schließlich über die Semantik „Tourismus als Wirtschaftszweig“ eine Konzeptionalisierung dieses Phänomens als Wirtschaftszweig, während die Diskussion und Entwicklung anderer Konzepte durch den Tourismus-Ausdruck unterdrückt werden. Sind Gesundheitssysteme beziehungsweise Gesundheitsversorgung erst einmal als Wirtschaftszweig konzeptionalisiert, werden dabei nicht nur medizinische Behandlungen als Dienstleistung in einem gewinnorientierten System determiniert, sondern darüber hinaus auch Patientinnen als Kundinnen festgeschrieben. Der Ausdruck Medizintouristin folgt diesem Konzept der Patientin als Kundin beziehungsweise Konsumentin.

\subsubsection{TOURISTIN - KUNDIN - PATIENTIN?}

Um zu verstehen, in welchem Maße zu medizinischen Zwecken Reisende den unterschiedlichen Begriffen von Touristin, Kundin oder Patientin zugeordnet werden können, werden verschiedene Arbeiten herangezogen. Zum einen wird unter Berücksichtigung der Perspektive von Tourismuswissenschaftlerinnen gezeigt, welche Eigenschaften beziehungsweise welches Verhalten für Touristinnen kennzeichnend sind. So beschreibt zum Beispiel der Tourismuswissenschaftler Metin Kozak in seinem Aufsatz das typische Verhalten von Touristinnen anhand eines DreiPhasen-Modells. Dieses wird kurz vorgestellt und überprüft, inwiefern es sich auf reisende Patientinnen übertragen lässt. Zum anderen wird auf die Betroffenenperspektive eingegangen. Da es 
in der Forschung um transnationale Medizin nur sehr wenige Studien mit Betroffenen gibt, wird zusätzlich die Arbeit der Sozialwissenschaftlerinnen Seibel et al. herangezogen, die in einer qualitativen Interviewstudie Palliativpatientinnen zu ihrem Selbstverständnis befragten. Sie legten in ihrer Befragung den Fokus auf die Unterscheidung zwischen Kundin und Patientin. Diese Studie ist also gut dazu geeignet, die von den Betroffenen als wichtig empfundenen Konnotationen dieser beiden Ausdrücke in den Blick zu nehmen.

Der Tourismuswissenschaftler Kozak erkennt die Bedeutung von Tourismus als Wirtschaftszweig und bedient sich in seiner Forschung ursprünglich sozialwissenschaftlicher Verhaltensmodelle für Konsumentinnen. Diese gehen von drei Hauptverhaltensstadien aus: prepurchase, consumption und postconsumption. Diese Phasen lassen sich auf das Verhalten von Touristinnen übertragen. Die erste Phase beginnt nach Kozak mit dem Wunsch beziehungsweise dem Bedürfnis, in den Urlaub zu fahren. Aus diesem Bedürfnis heraus begebe sich die Touristin auf Informationssuche und wäge die sich dabei ergebenden Alternativen gegeneinander ab (prepurchase). Die nächste Phase umfasse die Reise selbst, die Touristin entdecke und erlebe den Zielort und die damit verbundenen Produkte (consumption). In der dritten Phase verarbeite und beurteile die Touristin das Erlebte (postconsumption) (Kozak und Decrop 2009).

Versucht man, dieses Verhaltensmodell auf Personen zu übertragen, die zu medizinischen Zwecken ins Ausland reisen, ergeben sich gravierende Unterschiede. Solche Personen folgen nicht dem Wunsch, in den Urlaub zu fahren, sondern suchen eine medizinische Behandlung. Wie bereits beschrieben, würden es die meisten Patientinnen bevorzugen, die im Ausland gesuchte Behandlung nahe des Heimatortes zu erhalten, wenn sie nicht zum Beispiel wegen eines schlechten Versicherungsstatus dazu gedrängt würden, ins häufig kostengünstigere Ausland auszuweichen (Horowitz und Rosensweig 2007).

Bevor Patientinnen sich über mögliche Reiseziele informieren, haben sie zumeist bereits im Heimatland Informationen zu einer bestimmten medizinischen Behandlung eingeholt. Erst die Erkenntnis, dass die gesuchte medizinische Behandlung am Heimatort für die Patientin nicht verfügbar ist, führt zu der Entscheidung, zu reisen. Dies entspricht nicht der von Kozak beschriebenen prepurchase-Phase, der von Anfang an das Bedürfnis, zu verreisen, zugrunde liegt. Darüber hinaus haben Hudson und Culley in einer qualitativen Interview-Studie ermittelt, dass es Patientinnen und Patienten, die aus reproduktionsmedizinischen Gründen reisen, in den wenigsten Fällen um die Suche nach etwas Exotischem geht; die Betroffenen suchen im Gegenteil häufig nach Zielen, die nahe am Heimatort oder bereits aus anderen Aufenthalten bekannt und im besten Falle vertraut sind: 
Feeling comfortable with the place to which one was travelling was an important consideration for many in the present study. Participants often reported having been to their chosen destination on holiday or through their work, having family or friends there, or, with less familiar places, feeling that in being Western it was in some way at least culturally familiar: 'Familiarity of the city, having a friend there who lives in [the USA] who had done it, made a point, and the fact that I could be there every other week ... So we would literally call [the USA] our second home for about two years' (Deborah). Other participants had explicitly ruled out certain countries as being too ,foreign' (Hudson und Culley 2011, S. 577).

Auch am Beispiel der Explikation von Tourismus des Ethnologen Ronald Lutz lassen sich deutliche Unterschiede zwischen Touristinnen und zu medizinischen Zwecken Reisenden aufzeigen. Lutz charakterisiert Tourismus als den „Traum des nicht gelebten, aber doch ersehnten Lebens.“ Die während des Urlaubes erlebte Welt stelle sich als Spiel dar, das nach dem Aufenthalt als beendet betrachtet werde (Lutz 1992, S. 233). Der unerfüllte Wunsch nach einem Kind, der mittels einer reproduktionsmedizinischen Behandlung möglicherweise Wirklichkeit wird, kann nicht als Spiel verstanden werden, das nach dem Aufenthalt beendet ist. Dies gilt womöglich noch mehr für jene Frauen, die wieder nach Haus fahren, ohne schwanger geworden zu sein. Im Ausland durchgeführte medizinische Behandlungen, seien es eine Gelenkoperation, eine Organtransplantation oder eine reproduktionsmedizinische Behandlung, haben gravierende Auswirkungen auf den Alltag und dienen nicht einer kurzfristigen Befriedigung von Sehnsüchten.

Zwar gehen auch Patientinnen als Reisende häufig touristischen Aktivitäten nach. Dies ist jedoch kein Beweis dafür, dass sie vorrangig als Touristinnen zu betrachten sind. So würde man zum Beispiel auch Geflüchtete nicht als Touristinnen bezeichnen, weil sie für ihre Flucht die kommerziellen Angebote einer Fluggesellschaft nutzen. Das Beispiel der Geflüchteten verdeutlicht, dass das bloße Vorhandensein von vergleichbaren Verhaltensweisen keine Schlussfolgerungen über die Lebenswirklichkeit der Betroffenen erlaubt. So kann der Ausdruck Tourismus die Wirklichkeit der reisenden Patientinnen nicht erfassen, da die Semantik des Ausdrucks Aspekte wie Leid, finanziellen Druck oder medizinische Behandlung nicht einschließt. Eine Metapher betont immer bestimmte Inhalte des beschriebenen Phänomens auf Kosten anderer. In Bezug auf die Situation der reisenden Patientinnen wird zum Beispiel durch die Metapher Tourismus der Freizeitaspekt deutlicher hervorgehoben als der Leidaspekt. Leiden ist nicht nur Teil der Wirklichkeit der Patientinnen, sondern häufig der Antrieb für die Reise. In einem Aufsatz über Stammzelltourismus macht Norra MacReady z. B. auf die verzweifelte Lage der Reisenden aufmerksam: von "desperately ill patients and their families go to clinics all over the world in the hope of finding at least an improvement in their condition, if not a cure." (MacReady 2009, S. 317) (Hervorhebungen durch M.M.).

Auch Smith geht auf Betroffene ein, die den Ausdruck Tourismus als überaus unangemessen empfinden, da er das von ihnen erlebte Leiden in keiner Weise berücksichtigt, ja sogar verhöhnt: 
The partner of an American that travelled to India for heart surgery due to a lack of health insurance coverage at home commented in a hearing before the United States Congress, 'we were not tourists seeking an inexpensive, exotic vacation while having medical treatment. We were fighting for Howard's life'. The contention being that the label 'tourism' suggests a light-heartedness that disregards the trauma involved for many engaging in this kind of activity" (Smith 2012, S. 2-3).

Wer für das Leben einer geliebten Person kämpft, muss tatsächlich den Eindruck haben, seine Situation werde durch die Semantik Freizeit des Ausdrucks Tourismus verkannt. Auf der anderen Seite wird die Diskrepanz zwischen realem, schwerem Leid und den Freizeitassoziationen des Begriffs von einigen Autorinnen genutzt, um das Handeln der Patientinnen polemisch zu beschreiben. Indem die Reisenden als „Transplantationstouristinnen“ oder „Sterbetouristinnen“ bezeichnet werden, wird ihr Anliegen diskreditiert. Im gleichen Sinne wird auch das semantische Feld Wirtschaft dazu genutzt, kommerzielle Strukturen hervorzuheben und polemisch darzustellen. Über die Konnotation Wirtschaftszweig knüpft der Tourismus-Ausdruck im medizinischen Kontext an die in den letzten Jahren geführte Diskussion über die Ökonomisierung des Gesundheitswesens an. Mit Hilfe des Ausdrucks können zum Beispiel die Privatisierung von Krankenhäusern, die Pflege als ökonomisierte Form der Dienstleistung und nicht zuletzt das Verständnis von Patientinnen als Kundinnen oder Konsumentinnen implizit legitimiert werden.

Die Medizinethik diskutiert schon seit einiger Zeit, ob Patientinnen nicht besser als Kundinnen verstanden werden sollten, um sich so von einer traditionellen Auffassung des Ärztin-PatientinVerhältnisses abzugrenzen. Denn lange Zeit herrschte in der Medizin ein paternalistisches Selbstverständnis der Ärztin vor. In der Diskussion über mehr Selbstbestimmung von Patientinnen scheint dagegen der Ausdruck Kundin dazu geeignet, das Recht auf Autonomie der Patientinnen hervorzuheben und diese darin zu bestärken, es auch einzufordern. Eine Studie von Seibel et al. mit Patientinnen aus der Palliativmedizin hat diese These untersucht. Sie ermittelten fünf unterschiedliche Typen von Kranken, drei davon beschreiben sich selbst eher als Patientinnen, zwei als Kundinnen. Auffällig ist, dass diejenigen, die sich selbst als Kundinnen beschreiben, in ihrer Ausdeutung des Ausdrucks tatsächlich auf die positiv konnotierten Aspekte wie Stärkung der Autonomie und Kundenorientierung verweisen. Deshalb stellen Seibel et al. fest, „dass die Rede vom Patienten als Kunde nur Ausdruck des Wunsches nach individualisierter Behandlung und Selbstbestimmung des Patienten“ sei (Seibel et al. 2013, S. 4). Doch unabhängig davon, ob die Palliativpatientinnen sich als Kundinnen oder Patientinnen verstanden, zeigten sich alle der Asymmetrie des Ärztin-Patientin-Verhältnisses bewusst. Denn gestern wie heute ist das Ärztin-Patientin-Verhältnis geprägt von der Bedürftigkeit der Patientin einerseits und dem Fachwissen der Ärztin andererseits.

Dabei entspringt die Fürsorgepflicht der Ärztin einem Berufsverständnis, das auch heute noch von der Sorge um das Wohl der Patientin aus altruistischen Motiven geprägt ist. Auch Kun- 
denorientierung hat zwar im besten Falle tatsächlich die Zufriedenheit von Kundinnen zum Ziel, dahinter steht aber im Kontext eines liberalen Marktes oft auch nur ein Profitmotiv. Dieser negative Aspekt wird häufig übersehen, wenn der Ausdruck Kundin statt Patientin zum Zwecke der Hervorhebung der Selbstbestimmung genutzt wird. Kundin als Bezeichnung für die Patientin macht aus der behandelnden Ärztin eine Dienstleisterin. Zwar kann eine Dienstleistung in der Medizin tatsächlich als Dienst an der Patientin verstanden werden. Doch ist es darüber hinaus ein in der Wirtschaft üblicherweise verwendeter Ausdruck, der auf Gewinnorientierung und Profitsteigerung verweist. Natürlich müssen auch Ärztinnen für ihre Leistungen entlohnt werden, dies sollte aber zum einen in angemessenem Rahmen geschehen. Zum anderen sollte das Ziel der Behandlung jeder Patientin die Linderung der Beschwerden mit den dazu am besten geeigneten Mitteln sein, unabhängig von der Höhe der Entlohnung der Ärztin oder der Gewinnspanne, die mit der Behandlung zu erzielen ist. Versteht man in Folge des Konzeptes Patientin als Kundin Ärztinnen als Dienstleisterinnen, wird eine Fremd- und Selbstwahrnehmung der Ärztin befördert, die monetäre Interessen in den Fokus der Behandlungsmotivation rücken. Dies kann das Vertrauen der Patientinnen in das Handeln ihrer Ärztin erschüttern. Die Verwendung des Begriffs Kundin kann so die Asymmetrie des Ärztin-Patientin-Verhältnis verstärken, entgegen der Intentionen vieler Autorinnen, die damit zunächst die Position der Patientin stärken wollten. Dieses Beispiel zeigt einmal mehr, wie sorgfältig wir mit den Ausdrücken, die wir nutzen, umgehen sollten.

Im ethischen Diskurs ist es selten hilfreich, zur Steigerung der Überzeugungskraft auf implizite Wertungen oder Botschaften durch Metaphern zu setzen. Eine Metapher hat immer zahlreiche Ausdeutungsmöglichkeiten und es ist nahezu unmöglich, diese vollständig im Vorfeld zu überblicken. Die Analyse der Verwendung des Ausdrucks Medizintourismus in der ethischen Debatte hat deutlich gezeigt, dass die unterschiedlichen Diskursteilnehmenden die schillernden Verständnisweisen des Ausdrucks für ihre Zwecke nutzen und ein Ausdruck die an seine Semantik gebundenen Denkrichtungen vorgibt. Die Konzeptionalisierung von Patientinnen als Kundinnen fügt sich als schlüssige Metapher in die Debatte um die Ökonomisierung von Gesundheitsversorgung ein. Die Verwendung des Ausdrucks Medizintourismus unterstützt durch die Semantik von Tourismus als Wirtschaftszweig diese ökonomisierte Sichtweise. Dabei erweist sich die konstitutive Funktion der Metapher als besonders problematisch: Die Ökonomisierung wird als gegeben und unumgänglich angesehen, und Alternativen werden so gar nicht erst gedacht.

Zusammenfassend konnte in diesem Kapitel gezeigt werden, dass es in der transnationalen Medizin durchaus inhaltliche Überschneidungen der Begriffe von Touristin und Patientin gibt. Unter Berücksichtigung aller Konnotationen einerseits und Selbstbeschreibungen von Betroffenen 
andererseits muss jedoch festgehalten werden, dass im Verhältnis zu den wenigen inhaltlichen Überschneidungen die unpassenden Konnotationen überwiegen. Die Verfügbarkeit und die Qualität einer medizinischen Behandlung bestimmen vorrangig sowohl die Reisetätigkeit an sich als auch die Entscheidung für einen bestimmten Zielort. Während für eine Touristin die Reise Zweck an sich ist, ist die Reise für die aus medizinischen Gründen reisende Person lediglich ein Mittel zum Zweck, der Zweck ist die medizinische Behandlung. 


\section{AUf DER SUCHE NACH EINEM ALTERNATIVEN AUSDRUCK}

Aufgrund der bis hierhin geleisteten Arbeit und insbesondere unter Berücksichtigung der Selbstbeschreibung der Betroffenen muss man zu dem Schluss kommen, dass eine Einordnung von zu medizinischen Zwecken Reisenden in das Phänomen Tourismus nicht adäquat ist. Zwar gibt es einige Definitionen von Tourismus, die formal auch zu medizinischen Zwecken reisende Personen einschließen. So zum Beispiel die Definition der WTO, die besagt, Tourismus umfasse alle „Aktivitäten von Personen, die an Orte außerhalb ihrer gewohnten Umgebung reisen und sich dort zu Freizeit-, Geschäfts- oder bestimmten anderen Zwecken nicht länger als ein Jahr ohne Unterbrechung aufhalten“ (World Tourism Organization 1995). Medizinische Behandlungen könnten unter „bestimmte andere Zwecke“ also leicht in das Phänomen Tourismus eingeordnet werden. In dieser Arbeit konnte ich jedoch zeigen, dass der Ausdruck Medizintourismus implizit Wertvorstellungen unterschiedlichster Art transportiert: Nachdem für den Ausdruck Tourismus die drei semantischen Hauptaspekte Ortswechsel, Freizeit, Wirtschaftszweig herausgearbeitet wurden, konnte bewiesen werden, dass diese semantischen Felder im Vokabular von Texten, in denen der Ausdruck Medizintourismus verwendet wird, bedient werden. Über die Semantik von Tourismus entwickelt die Metapher Medizintourismus ihre illustrative, innovative und konstitutive Funktion. Alle diese Funktionen haben normatives Potential. So beeinflusst die Metapher nicht nur unsere Wahrnehmung von dem Phänomen zu medizinischen Zwecken Reisender, sondern auch dessen Bewertung. Allein durch die Verwendung der Metapher Medizintourismus werden Werturteile von den Autorinnen teils polemisch und ohne klare Argumentation vorgebracht. Deshalb komme ich aus diskursethischer Perspektive zu der Einschätzung, dass der Ausdruck Medizintourismus der in der interdisziplinären Bioethik geforderten sachlichen Diskussion nicht angemessen ist.

Im folgenden Kapitel werden zunächst Kriterien für einen besseren Ausdruck herausgearbeitet. Daran anschließend werden unterschiedliche Vorschläge aus der ethischen Debatte für alternative Ausdrücke vorgestellt und deren Eignung für eine bessere Auseinandersetzung mit dem Phänomen diskutiert.

\subsection{ANFORDERUNGEN AN EINEN ALTERNATIVEN AUSDRUCK}

Wenn nun Medizintourismus als Bezeichnung für das Phänomen zu medizinischen Zwecken Reisender ausgedient hat, welchen Ausdruck sollen wir stattdessen verwenden? Ein besserer Ausdruck sollte dazu dienen, die Diskussion sachlicher zu gestalten und diese für neue Ideen und 
eine umfassende Argumentation insbesondere ethischer Fragestellungen zu öffnen. Er muss beschreibend sein, ohne das darzustellende Phänomen auf einzelne Aspekte zu reduzieren.

Auf der Suche nach einer neuen konzeptionellen Grundlage für das Verständnis von „fertility tourists“ schlagen die Soziologinnen Nicky Hudson und Lorraine Culley vor, „fertility tourists“ nach Sartre und Young in Serialitäten und nicht in einer homogenen Gruppe zu konzeptionalisieren (Hudson und Culley 2011). Das Serialitätskonzept, erstmals von Jean-Paul Sartre beschrieben und unter anderem von Iris Marion Young weiterentwickelt, besagt, dass Personen nicht in homogene Gruppen geteilt werden können, sondern als einzelne Individuen immer mehrere unterschiedliche Kriterien erfüllen, die sie unterschiedlichen Gruppen zuordnen lassen (Sartre 1980); (Young 1994). Durch Zuordnungen zu einer bestimmten Gruppe werden Individuen häufig auf ein bestimmtes Merkmal reduziert. So kann eine Person offensichtlich gleichzeitig, groß, intelligent, weiblich und weiß sein und ist damit mindestens diesen vier Gruppen zuzuordnen. Welche Eigenschaft oder welches Rollenbild dabei im Vordergrund steht, kann variieren und hängt entscheidend von der jeweiligen Situation ab. Das Serialitätskonzept soll also ermöglichen, möglichst viele Aspekte und Facetten einer Person erfassen zu können. Genau das sollte ein neuer Ausdruck für das bisher als Medizintourismus bezeichnete Phänomen leisten.

Hudson und Culley erläutern, das Serialitätskonzept diene dazu, Unterschiede und Gemeinsamkeiten besser untersuchen und voneinander getrennt betrachten zu können. Darüber hinaus ermögliche dieses Konzept, die Beteiligten unterschiedlichen Serialitäten zuzuordnen, beziehungsweise einen Wechsel zwischen Serialitäten anzuerkennen. Dies entspreche am ehesten der Selbstsicht der Betroffenen, die sich in einer von Hudson und Culley durchgeführten Studie zu unterschiedlichen Zeitpunkten unterschiedliche Bezeichnungen gegeben hatten, zum Beispiel Patientin, Unterstützerin, Elternteil (Hudson und Culley 2011). Die unterschiedlichen Rollen, in denen sich Betroffene sehen, sind ein Beispiel für die Vielfältigkeit des Phänomens auf der personellen Ebene. Im Phänomen der zu medizinischen Zwecken Reisenden sind gleichzeitig die strukturelle Ebene von Gesundheitsversorgung sowie die Ebene der unterschiedlichen Fachrichtungen mit ihren eigenen ethischen und rechtlichen Fragestellungen von großer Bedeutung, um den Facettenreichtum des Phänomens nur anzudeuten.

Weiterhin ermöglicht das Serialitätskonzept der Wissenschaftlerin, sich von einer einschränkenden Sichtweise beziehungsweise Stigmatisierung eines Phänomens oder von Personen zu distanzieren. Es kann also dazu dienen, neue und nicht zu dem Ur-Konzept passende Aspekte zu beleuchten. Trotzdem kommt man nicht umhin, das kennzeichnende Merkmal der Serialität - in diesem Fall die Reise für eine reproduktionsmedizinische Behandlung - zu benennen, dies sei in diesem Fall mit fertility tourism beziehungsweise ,fertility travel' geschehen (Hudson und Culley 
2011). In diesem Punkt übersehen die Autorinnen jedoch die konzeptionalisierenden Auswirkungen des Audrucks tourism. Dennoch halte ich den Verweis auf das Serialitätskonzept für zielführend, da es zwei wichtige Kriterien zum Ausdruck bringt: Ein neuer Ausdruck sollte erstens offen genug sein, um den unterschiedlichen Facetten des Phänomens gerecht werden zu können, zweitens muss er spezifisch genug sein, also mindestens ein verbindendes Element konkret benennen. Auf der Suche nach einem alternativen Ausdruck möchte ich also das Serialitätskonzept berücksichtigen, um einen Ausdruck zu finden, der einer sachlichen ethischen Diskussion über das Phänomen zu medizinischen Zwecken Reisender gerecht wird.

Welche Kriterien müsste ein Ausdruck erfüllen, um dem Serialitätskonzept zu entsprechen? Ideal wäre ein neues Wort. Ein neuer Ausdruck, der wertfrei ist und mit allen Inhalten gefüllt werden kann, die zu dem Phänomen dazugehören. Die Textanalyse hat gezeigt, dass der Bedarf besteht, Hyponyme zu schaffen, um so zum Beispiel Fachbereiche voneinander zu differenzieren und das Phänomen zu kategorisieren. Denn offensichtlich variieren auch die ethischen Fragestellungen je nach Fachbereich. So geht es im chirurgischen Bereich unter anderem um die mangelnde Absicherung von Patientinnen durch Versicherungen, im Bereich der Reproduktionsmedizin stehen zusätzlich rechtliche Beschränkungen im Fokus der Diskussion. Allen Fachbereichen gemein ist die Frage nach dem angemessenen Zugang zu bestimmten medizinischen Behandlungen und die damit verbundene Diskussion über Verteilungsgerechtigkeit. Ideal wäre es also, einen Ausdruck zu finden, der durch Adjektive erweiterbar ist, so dass das zur Diskussion stehende Gebiet eingegrenzt werden kann. Diese Adjektive können zum Beispiel für Fachbereiche, rechtliche Rahmenbedingungen, Regionen etc. gewählt werden (reproductive/transplant/surgical, European/US/Indian/worldwide, legal/illegal). Durch unterschiedliche Präzisierungen des Ausdrucks könnte dieser seinen grundsätzlich neutralen Charakter behalten und die Diskussion dennoch kreativer und vielfältiger gestaltet werden.

Sprache funktioniert jedoch nicht so, dass wir uns einfach neue Wörter ausdenken und diese dann sofort in den allgemeinen Sprachgebrauch übergehen. Ein berühmtes Beispiel für den gescheiterten Versuch, ein Kunstwort in den Sprachgebrauch einzuführen ist das Wort „sitt“, das in einem unter anderem vom Duden veranstalteten Wettbewerb dazu erkoren wurde, die Sprachlücke für „jemand hat genug getrunken und ist nicht mehr durstig“ zu füllen, so wie der Ausdruck satt für den vergleichbaren Zustand der Esserin nach dem Essen. Dennoch sollte der Versuch unternommen werden, einen möglichst neutralen und offenen Begriff für dieses Phänomen zu finden, der die Produzentinnen dazu auffordert, ihre Argumente zu dem Thema explizit zu machen. 


\subsection{VORSCHLÄGE AUS DEM DISKURS}

Dass der Versuch, einen neuen Ausdruck in einen Diskurs einzuführen, erfolgreich sein kann, zeigt sich in der fachlichen Debatte um reisende Patientinnen zu reproduktionsmedizinischen Zwecken, in dem sich der Ausdruck cross-border reproductive care in den letzten Jahren mehr und mehr durchgesetzt hat. Nahezu alle alternativen Begriffe beziehen sich auf den Bereich der Reproduktionsmedizin. Bereits 2002 forderte Pennings die Verwendung des Ausdrucks reproductive travelling anstelle von reproductive tourism. 2004 folgte der Vorschlag cross-border reproductive care (CBRC), der 2008 von der European Society of Human Reproduction and Embryology (ESHRE) aufgenommen wurde und sich seitdem in der medizinethischen Debatte um Reisende zu reproduktionsmedizinischen Zwecken durchzusetzen scheint. In der Diskussion über einen geeigneten Ausdruck plädierte Matorras 2005 hingegen für den Ausdruck reproductive exile.

Inhorn und Gürtin fassen die Diskussion über den Ausdruck reproductive tourism versus crossborder reproductive care in ihrem Artikel „Cross-border reproductive care: a future research agenda“ zusammen. Demnach plädierten einige CBRC Scholars dafür, an dem Ausdruck Tourismus festzuhalten, da er dazu diene, kritisch und analytisch die ökonomischen Infrastrukturen des Phänomens zu erfassen. Als weitere Vorschläge aus der Diskussion erwähnten Inhorn und Gürtin traffic, transnational circumvention, infertility journeys, global egg trade, eggs-ploitation, reproductive opportunism bis hin zu the new sex trade (Inhorn und Gürtin 2011). Es fällt auf, dass es sich bei all diesen Vorschlägen um Metaphern handelt.

Inhorn selbst hatte 2009 zusammen mit Patrizio dafür plädiert, den Vorschlag exile, ebenfalls eine Metapher, von Matorras aufzunehmen:

Reproductive exile provoked by restrictive reproductive laws and other arenas of constraint compounds the human tragedy of infertility. It is hoped that future empirical research with individuals and couples subjected to such temporary but painful exiles will spur policy-makers to address this important issue (Inhorn und Patrizio 2009, S. 906).

Vergleicht man tourism und exile, so ist vor allem der Begriff der Fremde unterschiedlich konnotiert: In Tourismus ist die Fremde positiv besetzt, da eine freiwillig unternommene Reise unterstellt wird, Exil hingegen beschreibt die Fremde als erzwungenen Ort und ist damit negativ besetzt; die betroffene Person ist Opfer einer bestimmten (meist politischen) Situation und hat den Aufenthalt außerhalb des Heimatlandes nicht frei gewählt. Bei Exil handelt es sich um einen Ausdruck, der auf die Perspektive der Patientinnen fokussiert. Bei allen von Inhorn und Gürtin gesammelten Ausdrücken handelt es sich um Metaphern, die mit den Assoziationen, die sie hervorrufen, spielen. Die Autorinnen stellen deshalb fest: „Most of this vocabulary is intentionally critical, suggesting that the more neutral term of CBRC fails to capture completely the complicat- 
ed, nuanced and variable aspects of reproductive travel“ (Inhorn und Gürtin 2011). Den Ausdrücken ist nicht nur eine einseitige Interpretation des Phänomens, sondern auch eine implizite Wertung der Situation gemein. Auch exile ist in diesem Sinne ein stark positionierender Ausdruck.

Ich halte es deshalb nicht für sinnvoll, den Vorschlag von Matorras, unterstützt von Inhorn und Patrizio, tourism durch exile zu ersetzen, anzunehmen. In dieser Arbeit wurden die komplexen Wirkungsweisen einer Metapher im Zusammenhang mit der Konzeptionalisierung des mit dem Ausdruck Medizintourismus bezeichneten Sachverhaltes aufgezeigt. Die Metapher ist ein Mittel, „das unser Konzeptsystem und die von uns vollzogenen Arten von Alltagsaktivitäten strukturiert. Es ist natürlich vernünftig anzunehmen, dass Worte allein Realität nicht verändern. Doch Veränderungen in unserem Konzeptsystem verändern auch das, was für uns real ist, und nehmen Einfluss darauf, wie wir die Welt wahrnehmen und wie wir nach diesen Wahrnehmungen handeln" (Lakoff und Johnson 2004, S. 168). Metaphern können also dem Neutralitätskriterium nicht standhalten. Um eine sachliche Diskussion zu befördern, ist es nicht zielführend, eine Metapher durch eine andere zu ersetzen.

Ist hingegen der Ausdruck cross-border reproductive care dazu geeignet, die Diskussion wertfreier und sachlicher zu führen? Rein sprachlich ließe er sich leicht auf andere Fachbereiche übertragen, und auch als Hyperonym hat er schon Verwendung gefunden. So führten Vallejo et al. eine Studie zu „cross-border care“ durch. Sie verzichteten in ihrem Artikel „Volume and diagnosis: an approach to cross-border care in eight European countries“ ganz auf die Verwendung von Ausdrücken wie medical tourism. Care stellt keine Metapher dar, sondern kann in seinem ursprünglich gemeinten Sinn - zum Beispiel Behandlung, Versorgung - verstanden werden. Dennoch handelt es sich bei care nicht um einen neutralen Ausdruck. Blyth et al. kritisieren den Ausdruck cross-border reproductive care als „value-laden“, da care eine fürsorgliche Versorgung der Patientinnen unterstelle, die in der Realität nicht gegeben sei. Sie schlagen hingegen crossborder reproductive services vor. Services beinhaltet allerdings in gleicher Weise wie tourism das semantische Feld Wirtschaft, und kann dadurch ebenso affirmativ verwendet werden. Weder cross-border reproductive care noch cross-border reproductive services sind also neutral beschreibende Ausdrücke und als Alternativen deshalb nicht geeignet.

Inhorn und Patrizio fordern, ein neuer Ausdruck solle die Mühen der Reisenden widergeben (Inhorn und Patrizio 2009). Da dies bereits einer Positionierung entspricht, halte ich dieses Kriterium nicht für sinnvoll. Anhand der Diskussion über einen alternativen Ausdruck im Bereich der Reproduktionsmedizin wird deutlich, dass viele Diskursteilnehmende mittels normativer Ausdrücke Wertungen vorgeben. Zwar kann es für die Diskussion durchaus bereichernd sein, 
wenn Kritik durch Neologismen verdeutlicht wird. Die Gefahr von implizit wertenden Metaphern besteht aber in der unreflektierten Weiterverwendung durch andere Diskursteilnehmende. Ein gutes Hyperonym für dieses komplexe Phänomen sollte diese Gefahr vermeiden und Raum für alle Facetten bieten. Das kann nur ein möglichst neutraler, beschreibender Ausdruck leisten.

Metaphern dienen der Kategorisierung von Sachverhalten. Deshalb sind sie gerade bei sich neu entwickelnden Phänomenen dazu geeignet, deren Inhalte und Strukturen zu erschließen und für die Diskussion aufzubereiten. Wenn eine Metapher bestimmte Aspekte hervorhebt und andere verbirgt, erklärt sie immerhin einen Teil des Phänomens. Dabei werden aber immer andere Aspekte in den Hintergrund gedrängt, die für die Erfassung und Bewertung des gesamten Phänomens ebenso wichtig sein können. Deshalb sollten wir uns für einen neuen Ausdruck nicht auf die Suche nach einer neuen Metapher machen, da auch diese wiederum nur Teilaspekte beleuchten wird.

Kristen Smith spricht sich aus einer kritisierenden Absicht heraus ausdrücklich für medical tourism als Hyperonym aus. Sie plädiert für eine Beibehaltung des Ausdrucks, weil Medizin und Tourismus intuitiv nicht miteinander vereinbar seien und sich der Ausdruck deshalb besonders gut eigne, um auf die ethische Problematik hinzuweisen:

An increasing number of academics have disputed the use of the word 'tourism' due to what is regarded as its ill-fitting, 'suggestion of leisure and frivolity' and lack of neutrality. However, it is precisely this reason that makes it entirely appropriate, as the coupling of the two terms directly highlights the bioethical concerns inherent in the industry. [...] Within this current neoliberal capitalist paradigm, the continued use and coupling of the terms 'medical' and 'tourism' can be seen as constructive as it serves to highlight the uncomfortable position and awkward self-conscious placement the industry occupies. It is here that the bioethical dimensions of the practice and industry are brought into a much sharper focus than could be achieved with a more benign, descriptor such as the term 'medical travel', which seems to be increasingly favoured by promoters within the industry and academia alike (Smith 2012, S. 2-3).

Smith übersieht dabei, dass die anderen Verwendungsweisen, zum Beispiel die affirmative Verwendung des Ausdrucks, das kritisierende Potential der Metapher untergraben. Sie hebt die kritisierende Funktion des Ausdrucks hervor. Für eine sachliche Diskussion der ethischen Aspekte ist es jedoch nicht zielführend, eine solche emotionalisierte und implizite Kritik zu fordern.

Angesichts dieser Anforderungen scheint global health auf den ersten Blick ein zutreffender Ausdruck zu sein. Er tauchte zwar in der ethischen Debatte bereits auf, wurde jedoch bisher nicht explizit als Alternative für medical tourism vorgeschlagen. Allerdings ist dieser Ausdruck inhaltlich vage und erfüllt damit das Spezifitätskriterium nicht. Ein neuer Ausdruck sollte mindestens ein kennzeichnendes Merkmal des Phänomens zu medizinischen Zwecken Reisender 
benennen. Anhand der analysierten Explikationen konnte sich als einzige gemeinsame inhaltliche Aussage der Ortswechsel von Personen herausgearbeitet werden. Das Kriterium des Ortswechsels ist allen Fachbereichen gemein und sollte sich deshalb im Ausdruck abbilden. Außerdem ist der Gesundheitsbegriff selbst in der Diskussion und zum Beispiel im Bereich der Reproduktionsmedizin und Schönheitschirurgie häufig umstritten. In jedem Fall handelt es sich aber um Inanspruchnahme von medizinischer Versorgung, so dass es naheliegend ist, dieses im Ausdruck auch anzuführen.

Ich schlage deshalb als Ausdruck Transnationale Medizin beziehungsweise transnational medicine vor. Denn dieser Ausdruck spiegelt zum einen die inhaltlichen Aspekte, die allen Bereichen gemein sind, wider. Zum anderen ist er sowohl neutral als auch flexibel genug, um auf alle Kontexte im Phänomen angewendet zu werden. Der Ausdruck transnational medicine ist gut geeignet, um ihn auf Teilbereiche zu spezifizieren und damit die bisher verwendeten Hyponyme zu ersetzen. So kann man zum Beispiel von transnationaler Reproduktionsmedizin sprechen statt von Reproduktionstourismus. Gleichzeitig kann er durch differenzierende Adjektive ergänzt werden. So ließe sich zum Beispiel illegal transnational transplantation (medicine) von legal transnational transplantation (medicine) unterscheiden.

Der neue Begriff regt zu Fragen an wie: Möchten wir eine transnationale Medizin nach den Gesetzen des öffentlichen Marktes? Oder sollen Versicherungssysteme und öffentliche Gesundheitsstrukturen einbezogen werden? Verstehen wir medizinische Versorgung als ein kommerzielles Gut oder als ein Menschenrecht?

Der wissenschaftliche Diskurs braucht solche neutralen Ausdrücke ohne normative Festschreibungen, um eine offene, vorurteilsfreie Diskussion über das Phänomen zu ermöglichen. Sie schaffen Raum für eine gründliche Auseinandersetzung mit der Frage, welche Strukturen betroffen sind - touristische ebenso wie solche der Gesundheitsversorgung - und damit eine Diskussion über die grundlegende Frage, ob medizinische Versorgung als Menschenrecht oder Ware aufzufassen ist. 


\section{SCHLUSSFOLGERUNG UND AUSBLICK}

Die vorliegende Arbeit schafft eine Grundlage dafür, die Diskussion über die ethischen Fragen der transnationalen Medizin im interdisziplinären Diskurs sachlicher zu gestalten. Die besondere Leistung dieser Arbeit besteht dabei darin, die ethische Analyse durch eine qualitative wie quantitative Auswertung einschlägiger Texte aus der fachlichen Debatte zu fundieren. So gelang es, bereits zuvor von verschiedenen Autorinnen geäußerte Kritik an dem Ausdruck Reproduktionstourismus zu belegen und darüber hinaus die Wechselwirkungen zwischen den Hyponymen aufzudecken und in den Kontext der Leitmetapher Medizintourismus zu setzen. Dabei ist von besonderer Bedeutung, dass erstmals in einer systematischen Untersuchung die unterschiedlichen Diskurse der verschiedenen Fachbereiche über Patientinnen, die für eine medizinische Behandlung reisen, vergleichend betrachtet wurden.

Die Analyse von 45 Texten aus der ethischen Debatte ergab, dass eine Vielzahl von Hyponymen zu dem Ausdruck Medizintourismus verwendet wird. Die Ausdrücke reichen dabei von Reproduktionstourismus über Sterbetourismus, Kosmetiktourismus bis hin zu Transplantationstourismus, um nur einige der Unterbegriffe zu nennen. Da jeder Fachbereich mit ganz anderen strukturellen, rechtlichen und ethischen Fragestellungen konfrontiert ist, werden auch die unterschiedlichen Hyponyme entsprechend unterschiedlich präzisiert und konnotiert. Teilweise widersprechen sich die Explikationen in ihren Aussagen sogar. Diese Uneinigkeit über die Bedeutungen des Ausdrucks spiegelt zum einen die Komplexität der transnationalen Medizin wider, sie verhindert aber auch eine sachliche Diskussion. Es konnte zum Beispiel gezeigt werden, dass das Vokabular von dem fachlichen Hintergrund der Autorinnen beeinflusst ist. Dies verdeutlicht, wie aufmerksam man mit der Ausdeutung und Verwendung von Metaphern in interdisziplinären Diskursen umgehen muss, da keineswegs Einigkeit über die impliziten Botschaften eines bestimmten Ausdrucks herrscht.

Die Analyse der Metapher in Anlehnung an Kovács` Metapherntheorie hat ergeben, dass die Metapher Medizintourismus über die illustrative, die innovative und die konstitutive Leistung eine normative Aussagekraft entfaltet. Dabei kann zwischen epistemischer und moralischer Normativität unterschieden werden. Während die epistemische Normativität die Wahrnehmung bestimmter Strukturen lenkt, weil diese durch den Ausdruck betont werden, werden über die moralische Normativität implizit Wertungen vermittelt. Das normative Potential der TourismusMetapher wird je nach Intention der Autorin unterschiedlich genutzt. Es konnte gezeigt werden, 
dass der Ausdruck sowohl pejorativ, euphemistisch, kritisierend als auch affirmativ verwendet wird. Dies hat zur Folge, dass der Begriff verwirrende und widersprüchliche Botschaften vermittelt. Durch die häufige Verwendung des Ausdrucks transplant tourism in Zusammenhang mit illegalem Organhandel kann es zum Beispiel zu der impliziten Annahme kommen, es handele sich auch bei anderen Reisen zu medizinischen Zwecken, zum Beispiel für eine reproduktionsmedizinische Behandlung, um illegale Vorgänge. Dies ist sachlich jedoch häufig nicht zutreffend. Über die Metapher erfolgt implizit eine Bewertung bestimmter Strukturen, die Botschaften sind dabei aber nicht eindeutig, da die Metapher unterschiedliche Wertungen zulässt.

Die normative Wirkkraft einer Metapher entfaltet sich über die Semantik des zugrunde gelegten Ausdrucks, in diesem Fall Tourismus. Anhand lexikaler Definitionen wurden für den Ausdruck Tourismus die drei semantischen Hauptaspekte Ortswechsel, Freizeit und Wirtschaft herausgearbeitet. In der Textanalyse wurde gezeigt, dass die drei semantischen Felder auch in der ethischen Debatte um die transnationale Medizin herangezogen werden. Am häufigsten wird dabei das semantische Feld Wirtschaft bedient, gefolgt von Ortswechsel und Freizeit.

Auffällig war dabei, dass sich die Häufigkeiten der Verwendungen der semantischen Felder im gesamten Korpus von den Häufigkeiten in den Explikationen unterscheiden. Das heißt: Während im gesamten Korpus das semantische Feld Wirtschaft am häufigsten bedient wurde, wurde es in den Explikationen am seltensten erwähnt. In den Explikationen wurde hingegen am häufigsten das semantische Feld Ortswechsel bedient. Daran lässt sich die epistemische Normativität der Metapher zeigen: Die Semantik gibt die Denkrichtung für die Erfassung bestimmter Strukturen vor. So wird über die Bedeutung von „Tourismus als Wirtschaftszweig“ auch die transnationale Medizin als Wirtschaftszweig verhandelt. Andere Aspekte, die der Ausdruck Tourismus nicht impliziert, werden hingegen in der Diskussion nicht berücksichtigt. Um dies zu überprüfen, wurde in dem Textmaterial die Anwendung des semantischen Feldes Leid untersucht. Tatsächlich fand es sich wesentlich seltener als die anderen semantischen Felder. Die Tatsache, dass Leid für viele Personen Ursache des Reisens ist, wird so unterdrückt.

Darüber hinaus wurde festgestellt, dass die Tourismus-Metapher es nahelegt, das Phänomen reisender Patientinnen in erster Linie als Wirtschaftszweig zu diskutieren. Die Verwendung des Ausdrucks Medizintourismus behindert so eine Diskussion über das Recht auf eine Gesundheitsversorgung in der Nähe des Heimatortes und fördert die Auffassung, die marktförmige Organisation von medizinischen Behandlungen in einem privaten Sektor sei normal. Der Ausdruck Tourismus billigt so nicht nur das Faktum als solches, sondern liefert gleichzeitig die Legitimation für ein gewinnorientiertes Verhalten. Über das semantische Feld Freizeit suggeriert er dabei Freiwilligkeit und Vergnügen der reisenden Patientinnen. Wichtige ethische Aspekte wie der 
Zugang zu medizinischer Versorgung und Fragen der Verteilungsgerechtigkeit werden kaum beachtet. Die Metapher befördert eine Konzeptionalisierung von medizinischer Versorgung als Wirtschaftszweig im Gegensatz zu einem auf solidarischen Prinzipien basierenden Gesundheitssystems unter der Prämisse von medizinischer Versorgung als Menschenrecht.

Zusammenfassend kann also festgehalten werden, dass die Metapher Medizintourismus in der ethischen Diskussion normativ verwendet wird. Dabei kann zwischen epistemischer und moralischer Normativität unterschieden werden. Aus diskursethischer Sicht ist besonders problematisch, dass beide Formen von Normativität implizit über den Ausdruck vermittelt werden. Eine gründliche ethische Debatte ist hingegen auf explizite Argumentation angewiesen. Die Verwendung von Metaphern mit ihren unterschiedlichen Wirkungsweisen und damit verbundenen Wechselwirkungen von Konnotationen, die bei dem Ausdruck Medizintourismus von pejorativ über kritisierend bis hin zu euphemistisch reichen, steht einer expliziten Argumentation im Weg. Dies sollte im Interesse einer transparenten und sachlichen ethischen Debatte vermieden werden.

Metaphern können zwar hilfreich sein, um bestimmte Sachverhalte zu illustrieren und Meinungen zu unterstreichen. Bei Medizintourismus handelt es sich jedoch um einen Ausdruck, der zur Leitmetapher eines außerordentlich komplexen Sachgebietes geworden ist, dem er durch seine einschränkende Betonung bestimmter Aspekte mittlerweile nicht mehr gerecht wird.

Ich komme also zu dem Schluss, dass die Verwendung der Metapher Medizintourismus in der ethischen Diskussion nicht angemessen ist. Ein Ausdruck, der ein komplexes Phänomen beschreibt, sollte neutral sein, um eine umfassende Diskussion der ethischen Aspekte zu ermöglichen. Gleichzeitig darf er nicht zu beliebig sein, er sollte den herausragenden Aspekt benennen. Ein neuer Ausdruck muss also beschreibend sein, ohne zu bewerten. Und er muss flexibel genug sein, um zum Beispiel durch ergänzende Adjektive bestimmte Zusammenhänge spezifizierend besprechen zu können. Vorschläge, die von Diskursteilnehmenden gemacht wurden, um den Begriff Medizintourismus zu ersetzen, wurden von mir diskutiert. Keiner der Vorschläge entsprach den Anforderungen Neutralität und Flexibilität. Ich schlage stattdessen die Bezeichnung transnationale Medizin vor. Dieser Ausdruck ist wertfrei, spiegelt die Bewegungen von Personen aus medizinischen Gründen wider und kann beliebig durch Adjektive erweitert werden, um ihn zum Beispiel auf spezifische Fachgebiete anzuwenden.

In dieser Arbeit habe ich mich auf die Auswirkungen der Metapher in der ethischen Diskussion konzentriert. Auf das Phänomen der transnationalen Medizin bezogen bleiben aber weiterhin 
viele Fragen offen: Welche Art der Gesundheitsversorgung wünschen wir uns lokal und global? Wieviel Ökonomisierung wollen wir zulassen?

Bisher wurden im Diskurs um die transnationale Medizin vor allem wirtschaftliche Strukturen thematisiert und auch kritisiert. Eine umfassende Diskussion zum Beispiel über Verteilungsgerechtigkeit hat bislang nicht stattgefunden. Über die gut begründete Einführung eines neuen Ausdrucks könnte eine Öffnung der Debatte in diese Richtung erfolgen. Nach all den Berichten über Ausbeutung, Kostenersparnis, mangelnden Zugang zu Gesundheitsversorgung von sowohl den Reisenden als auch den Bewohnerinnen der Zielländer muss die Debatte über eine globale Gesundheitsversorgung neu geführt werden. 


\section{ZUSAMMENFASSUNG}

In der vorliegenden Arbeit wurde die Anwendung des Terminus Medizintourismus in der ethischen Fachdebatte analysiert und insbesondere auf die normativen Auswirkungen seiner Verwendung hin untersucht.

Aus diskursethischer Perspektive stellt sich die Frage, inwieweit es für eine sachliche Diskussion sinnvoll und angemessen ist, die Metapher Tourismus im Zusammenhang mit zu medizinischen Zwecken Reisenden zu verwenden. Diese Frage wurde unter anderem mit Hilfe einer empirischen Untersuchung der tatsächlichen Verwendung der Metapher in der fachlichen Debatte beantwortet. Um überprüfen zu können, ob tatsächlich die Grundbedeutung der Metapher, nämlich Tourismus, die Debatte beeinflusst, musste zunächst die Semantik des Ausdrucks Tourismus herausgearbeitet werden. In einem nächsten Schritt wurden in einer systematischen Textanalyse Beiträge aus der Fachdiskussion ausgewertet. Dabei lag ein Schwerpunkt auf der Herausarbeitung unterschiedlicher Definitionen und Konnotationen der Metapher Medizintourismus. Die Auswertung der Texte erfolgte mithilfe der Software atlas.ti, die sowohl eine qualitative als auch zum Teil quantitative Analyse ermöglicht. Die Methode der Textanalyse orientierte sich an der Noticing-Collecting-Thinking-Methode von Susanne Friese.

In der anschließenden ethischen Diskussion konnte unter Berücksichtigung zuvor erarbeiteter Metaphertheorien dargelegt werden, inwiefern die Metapher Medizintourismus Einfluss auf die Inhalte der Diskussion nimmt. In Anbetracht der besonderen Anforderungen an eine interdisziplinäre Fachdebatte wurde kritisch untersucht, ob dieser Ausdruck für die ethische Fachdiskussion geeignet ist. Die Hypothese, dass durch die Verwendung der Metapher Medizintourismus implizit normative Botschaften vermittelt werden, wurde bestätigt und konnte anhand der Verwendungsweisen unterschiedlicher Ausdrücke wie Reproduktionstourismus und Transplantationstourismus eindrucksvoll aufgezeigt werden.

Die Ergebnisse führten insgesamt zu der Einschätzung, dass die Verwendung des Ausdrucks Medizintourismus in der ethischen Debatte nicht gerechtfertigt ist. Als Alternative schlage ich stattdessen die Verwendung des Ausdrucks Transnationale Medizin vor, da dieser Terminus dazu geeignet ist, die fachliche Debatte sachlicher zu gestalten und somit für die Diskussion neuer Aspekte zu öffnen. 


\section{LITERATURVERZEICHNIS}

Alleman BW, Luger T, Reisinger HS, Martin R, Horowitz MD, Cram P (2011): Medical tourism services available to residents of the United States. J Gen Intern Med 26, 492-497

Anderson FWJ, Wansom T (2009): Beyond medical tourism: authentic engagement in global health. Virtual Mentor 11, 506-510

Barclay E (2009): Stem-cell experts raise concerns about medical tourism. Lancet $\underline{373}$, 883-884

Bassan S, Michaelsen MA (2013): Honeymoon, medical treatment or big business? An analysis of the meanings of the term „reproductive tourism“ in German and Israeli public media discourses. Philos Ethics Humanit Med 8 , 1-8

Bauer JC (2009): Medical tourism: wave of the future in a world of hurt? Healthc Financ Manage 63, 36-38

Biggins SW, Bambha K, Terrault N, Inadomi J, Roberts JP, Bass N (2009): Transplant tourism to China: The impact on domestic patient-care decisions. Clin Transplant $\underline{23}$, 831-838

Black M (1954): Die Metapher. In: Haverkamp A (Hrsg.): Theorie der Metapher. Wissenschaftliche Buchgesellschaft, Darmstadt 1996, 55-79

Brockhaus Enzyklopädie online. Hrsg. v. Hünermann C; http://www.brockhausenzyklopaedie.de

Budiani-Saberi DA, Delmonico FL (2008): Organ trafficking and transplant tourism: a commentary on the global realities. Am J Transplant. $\underline{8}, 925-929$

Carabello L (2008): A medical tourism primer for U.S. physicians. J Med Pract Manage 23, 291294

Cohen IG (2010): Medical Tourism: The View from Ten Thousand Feet. Hastings Cent Rep $\underline{40}$

Donalies E: Die Wortbildung des Deutschen: Ein Überblick. 1. Auflage; G. Narr, Tübingen 2002

Encyclopaedia Britannica. http://www.britannica.com/EBchecked/topic/600773/tourism

Epstein M (2009): Sociological and ethical issues in transplant commercialism. Curr Opin Organ Transplant 14, 134-139

Evans RW (2008): Ethnocentrism Is an Unacceptable Rationale for Health Care Policy: A Critique of Transplant Tourism Position Statements. Am J Transplant $\underline{8}, 1089-1095$

Foster M, Mason M (2006): Outsourcing Health? Some Companies, Insurers Mull Sending Americans Abroad for Surgery. www.signonsandiego.com/news/health/20061102-0651outsourcinghealth.html

Fried BJ, Harris DM (2007): Managing healthcare services in the global marketplace. Front Health Serv Manage 24, 3-18

Friese S: Qualitative data analysis with ATLAS.ti. SAGE, London 2012

Gill J, Madhira BR, Gjertson D, Lipshutz G, Cecka JM, Pham P, Wilkinson A, Bunnapradist S, Danovitch GM (2008): Transplant tourism in the United States: a single-center experience. Clin J Am Soc Nephrol 3 , 1820-1828

Gupta AS (2008): Medical tourism in India: winners and losers. Indian J Med Ethics $\underline{5}, 4-5$ 
Healy C (2009): Surgical tourism and the globalisation of healthcare. Ir J Med Sci 178, 125-127

Horowitz MD, Rosensweig JA (2007): Medical tourism - Health Care in the Global economy. Physician Exec 6, 28-30

Howard-Jones N (1979): On the diagnostic term „Down's disease“. Med Hist 23, 102-104

Hudson N, Culley L (2011): Assisted reproductive travel: UK patient trajectories. Reprod Biomed Online 23, 573-581

Inhorn MC, Patrizio P (2009): Rethinking reproductive „tourism“ as reproductive „exile“. Fertil Steril $\underline{\text { 92, 904-906 }}$

Inhorn MC, Shrivastav P (2010): Globalization and reproductive tourism in the United Arab Emirates. Asia Pac J Public Health $\underline{22}$, 68-74

Inhorn MC, Gürtin ZB (2011): Cross-border reproductive care: a future research agenda. Reprod Biomed Online 23, 665-676

Jafar TH(2009): Organ trafficking: global solutions for a global problem. Am J Kidney Dis $\underline{54}$, $1145-1157$

Janich N: Pressemitteilung: Wahl des 23. „Unwort des Jahres“. http://www.unwortdesjahres.net/fileadmin/unwort/download/pressemitteilung_unwort2013. pdf; veröffentlicht am 14.01.2014; zuletzt geprüft am 07.06.2015

Jeevan R, Armstrong A (2008): Cosmetic Tourism and the burden on the NHS. J Plast Reconstr Aesthet Surg 61, 1423-1424

Jütte R: Alternative Medicine and Medico-Historical Semantics. In: Jütte R, Eklöf M, Nelson MC (Hrsg.): Historical aspects of unconventional medicine: Approaches, concepts, case studies. European Association for the History of Medicine and Health Publications, Sheffield 2001, 11-26

Kantor A (2008): Medical tourism takes off. AHIP Cover 49, 42-46

Knoll E: So weit gehen für ein Kind: Reproduktionstourismus als grenzüberschreitender Umweg. In: Bockenheimer-Lucius G, Thorn P, Wendehorst C (Hrsg.): Umwege zum eigenen Kind: Ethische und rechtliche Herausforderungen an die Reproduktionsmedizin 30 Jahre nach Louise Brown. Univ.-Verl. Göttingen, Göttingen 2008, 63-79

Kokubo A (2009): The interaction of the international society concerning kidney transplants--a consideration of diseased kidney transplants in Japan and transplant tourism over the world. Leg Med (Tokyo) 11 Suppl 1, 393-395

Kovács L: Medizin - Macht - Metaphern: Sprachbilder in der Humangenetik und ethische Konsequenzen ihrer Verwendung. Phil. Diss. Tübingen 2008; Lang, Frankfurt am Main 2009

Kozak M, Decrop A (Hrsg.): Handbook of Tourist Behavior: Theory and Practice. Routledge, New York 2009

Lakoff G, Johnson M: Leben in Metaphern: Konstruktion und Gebrauch von Sprachbildern. 4. Auflage; Carl-Auer-Systeme-Verlag, Heidelberg 2004

Leahy AL (2008): Medical tourism: the impact of travel to foreign countries for healthcare. Surgeon $\underline{6}, 260-261$

Lutz R: Der subjektive Faktor - Ansätze einer Anthropologie des Reisens. In: Kamer D, Lutz R (Hrsg.): Reisen und Alltag. Inst. für Kulturanthropologie und Europ. Ethnologie, Frankfurt/M. 1992, 229-273

MacReady N (2009): The murky ethics of stem-cell tourism. Lancet Oncol 10, 317-318

Matorras R (2005): Reproductive exile versus reproductive tourism. Hum Reprod 20, 3571 
MedRetreat: The Medical Tourist.

http://www.medretreat.com/medical_tourism/the_medical_tourist.html; zuletzt geprüft am 07.06.2015

Milstein A, Smith M (2006): America's new refugees - Seeking affordable surgery offshore. N Engl J Med 16, 1637-1640

Ministry of tourism India: Incredible India. http://incredibleindia.org/en/trade-nav/latestnews/1537-medical-tourism-in-india-22-march-2012; zuletzt geprüft am 07.06.2015

Mitka M (2009): Surgical tourism: some US patients travel abroad for less costly surgery. JAMA 302, 1519

Naraindas H, Bastos C (2011): Healing holidays? Itinerant patients, therapeutic locales and the quest for health. Anthropol Med 18, 1-6

Nucci Medical Clinic: http://nuccimedicalclinic.com/nmc-joins-the-medical-tourism-association; zuletzt geprüft am 07.06.2015

(NZZ) Neue Zürcher Zeitung online: "Sterbetourismus" ist das Schweizer Wort des Jahres. NZZ online, 5. Dezember 2007. http://www.nzz.ch/aktuell/panorama/wortdesjahres-schweiz1.594601; zuletzt geprüft am 07.06.2015

Olsen S (1991): GE-Präfigierungen im heutigen Deutsch: Ausnahmen von der ,Righthand Head Rule'? Beiträge zur Geschichte der deutschen Sprache und Literatur, 113, 333-366

Pafford B (2009): The third wave - medical tourism in the 21st century. South Med J 102,810 813

Pattinson SD (2008): Organ trading, tourism, and trafficking within Europe. Med Law 27, 191201

Pennings G (2002): Reproductive tourism as a moral pluralism in motion. J Med Ethics 28 , 337341

Pennings G (2005): Reply: Reproductive exile versus reproductive tourism. Hum Reprod 20, 3571-3572

Pennings G, Wert G, Shenfield F, Cohen J, Tarlatzis B, Devroey P de (2008): ESHRE Task Force on Ethics and Law 15: Cross-border reproductive care. Hum Reprod 23, 2182-2184

Pfeffer N (2011): Eggs-ploiting women: a critical feminist analysis of the different principles in transplant and fertility tourism. Reprod Biomed Online 23, 634-641

Qdays - Medical Holidays: http://www.qdays.com; zuletzt geprüft am 07.06.2015

Richards IA (1936): Die Metapher. In: Haverkamp A (Hrsg.): Theorie der Metapher. Wissenschaftliche Buchgesellschaft, Darmstadt 1996, 31-52

Sartre J-P: Kritik der dialektischen Vernunft: Band 1 Theorie der gesellschaftlichen Praxis. 9.-11. Tsd. Auflage; Rowohlt, Reinbek bei Hamburg 1980

Schweizer Wort des Jahres: http://www.chwort.ch; zuletzt geprüft am 07.06.2015

Seibel K, Krause F, Becker G (2013): Ärztliche Verantwortung gegenüber Palliativpatienten unter dem neuen Paradigma der Kundenorientierung. Ethik Med, online first: doi: 10.1007/s00481-013-0242-z.

Shibles WA, Maier B: The philosophy and practice of medicine and bioethics: A naturalistichumanistic approach. Springer, Dordrecht 2011

Shimazono Y (2007): The state of the international organ trade: a provisional picture based on integration of available information. Bull World Health Organ $\underline{85}$, 955-962 
Smith K (2012): The problematization of medical tourism: a critique of neoliberalism. Dev World Bioeth $\underline{12}, 1-8$

Srinivas R (2009): Exploring the potential for American death tourism. Michigan State University Journal of Medicine and Law $\underline{13}$, 91-122

Storrow RF (2005): Quests for conception: Fertility Tourists, Globalization and Feminist Legal

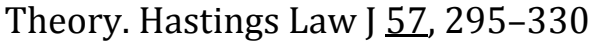

The Transplantation Society, International Society of Nephrology (2008): The Declaration of Istanbul: on Organ Trafficking and Transplant Tourism; verfügbar unter http://www.declarationofistanbul.org/about-the-declaration/structure-and-content; zuletzt geprüft am 07.06.2015

Thorn P (2008): Reproduktives Reisen. Profamilia. Verfügbar unter: http://www.profamilia.de/fileadmin/publikationen/Fachpublikationen/expertise_reproduktive s_reisen.pdf; zuletzt geprüft am 07.01.2015

Turner L (2008): ,Medical tourism' initiatives should exclude commercial organ transplantation. J R Soc Med 101, 391-394

Turner L (2009): „Dental tourism“: issues surrounding cross-border travel for dental care. J Can Dent Assoc 75, 117-119

Unti JA (2009): Medical and surgical tourism: the new world of health care globalization and what it means for the practicing surgeon. Bull Am Coll Surg 94, 18-25

World tourism organization (1995): Collection of tourism expenditure statistics; verfügbar unter https://pub.unwto.org/WebRoot/Store/Shops/Infoshop/Products/1034/1034-1.pdf; zuletzt geprüft am 07.06.2015

Young IM (1994): Gender as Seriality: Thinking about Women as a Social Collective. Signs $\underline{19}$ 713-738 


\section{DANKSAGUNG}

Mein besonderer Dank gilt Frau Prof. Dr. med. Claudia Wiesemann für die umfassende Betreuung meines Promotionsvorhabens. Ich bedanke mich insbesondere für die stets freundliche und konstruktive Kritik sowie für die oft auch kurzfristig eingeräumten Termine, die für das Weiterkommen so wichtig waren.

Bei allen Mitarbeiterinnen und Mitarbeitern des Instituts für Ethik und Geschichte der Medizin möchte ich mich für die angenehme Arbeitsatmosphäre im Institut bedanken. Die freundliche Aufnahme in das Team des Instituts während meiner Anstellung als wissenschaftliche Mitarbeiterin hat maßgeblich zu dem Gelingen dieser Arbeit beigetragen. 